\title{
Mechanisms Regulating Smooth Muscle Cell Phenotype by Atheroprone and Atheroprotective Hemodynamics on Endothelial Cells
}

\author{
A Dissertation \\ Presented to \\ the faculty of the School of Engineering and Applied Science \\ University of Virginia \\ in partial fulfillment \\ of the requirements for the degree
}

Doctor of Philosophy

by

Lauren Marie Boerboom

May

2013 
APPROVAL SHEET

The dissertation

is submitted in partial fulfillment of the requirements

for the degree of

Doctor of Philosophy

Lauren M. Boerboom

AUTHOR

The dissertation has been read and approved by the examining committee:

Brett R. Blackman, Ph.D.

Advisor
Richard Price, Ph.D., Chair

Norbert Leitinger, Ph.D.

Avril Somlyo, Ph.D.

Brian Wamhoff, Ph.D.

Accepted for the School of Engineering and Applied Science:

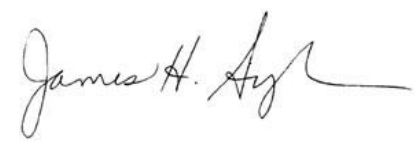

Dean, School of Engineering and Applied Science

May

2013 


\begin{abstract}
Atherosclerosis is an inflammatory disease of the arteries that develops preferentially in regions where shear stress, caused by blood flowing within the lumen of the vessel, is disturbed. Recent evidence demonstrates focal fibronectin (FN) expression in the subendothelial layer in pre-atherosclerotic and advanced lesions. We have found that atheroprone shear stress promotes FN deposition and inflammatory signaling pathways in endothelial cells (ECs). Recently, our lab discovered that EC platelet endothelial cell adhesion molecule-1 (PECAM), which is important for sensing biomechanical stresses, is necessary for the production, secretion, and assembly of the $\mathrm{FN}$-rich matrix in atheroprone regions.

Similar to ECs, underlying vascular smooth muscle cells (SMCs) also display a proinflammatory phenotype in regions of atherogenesis, which is a key feature involved in the development of atherosclerosis. Our lab has designed a novel in vitro system that applies human-derived shear stresses to ECs, which are co-cultured with underlying SMCs. This allows for molecular manipulation of individual cell types, while permitting the study of the inherent signaling cross-talk between ECs and SMCs. Such an approach is useful because, while atheroprone shear stress is known to regulate EC signaling and promote a pro-inflammatory environment, the influence of that signaling on SMC phenotype has yet to be investigated.

To assess the role of endothelial PECAM and FN signaling in promoting an inflammatory SMC phenotype, the co-culture model was exposed to atheroprone hemodynamic shear stress. siRNA knockdown of either PECAM or FN in ECs reduced SMC inflammatory genes, proteins, and transcription activity. The reduction in SMC inflammation also has the functional consequence of reducing monocyte adhesion. When EC FN is knocked down, the
\end{abstract}


inflammatory SMC phenotype was rescued through the addition of exogenous FN to the EC media. In addition to an increased inflammatory phenotype in response to atheroprone shear stress, SMCs are able to promote inflammation back to ECs. SMC FN was found to regulate EC inflammatory transcription activity and adhesion protein expression.

Next, the role of secreted proteins in signaling inflammation from ECs under atheroprone shear stress to SMCs was determined. Cell media collected from EC PECAM and FN knockdown experiments was able to differentially regulate SMC inflammation without the physical presence of ECs. A co-culture recovery experiment confirmed the ability for conditioned media from a control experiment to restore SMC inflammation despite EC FN knockdown. Media was then screened with a multiplexing approach for secreted cytokines differentially regulated by FN. Of the 19 cytokines measured, 12 were significantly inhibited by the knockdown of EC FN. In particular, GRO- $\alpha$ was found to increase SMC inflammation in a FN-dependent manner. This study supports the importance of cytokine signaling in promoting an atheroprone SMC phenotype downstream of the endothelial PECAM and FN pathway.

Finally, the atheroprotective role of Kruppel-like factor 4 (KLF4) in the endothelium was investigated. Anti-inflammatory and anti-thrombotic factors were previously shown to be under the regulation of the transcription factor KLF4. To study the role of KLF4 in establishing an atheroprotective phenotype, mono-cultured ECs were exposed to hemodynamic shear stress. KLF4 had increased expression under atheroprotective shear stress compared to atheroprone. Overexpression of KLF4 conferred an atheroprotective EC phenotype even when exposed to an atheroprone shear stress environment. In contrast, knockdown of EC KLF4 resulted in a proinflammatory and pro-thrombotic gene program despite exposure to atheroprotective shear 
stress. Therefore, the atheroprotective environment is not simply an absence of atheroprone stimulation, but actively promotes anti-atherogenic signaling pathways. 


\section{ACKNOWLEDGEMENTS}

This work would not have been possible without the support and encouragement of many people. My sincere gratitude is extended to:

- My advisor, Dr. Brett Blackman, for years of leadership and support.

- My committee, for their thoughtful advice and guidance.

- Devin Weinberg, my good friend and lab mate. Thank you for all you have taught me, for keeping lab fun, and for being there through this whole journey.

- Dr. Ryan Feaver, who has always been available and helpful. I appreciate all the brainstorming sessions, encouragement, and friendship over shared family dinners.

- Christin Hamilton, for technical expertise and advice, kind support, and above all, loyal friendship.

- Former Blackman lab members Dr. Brad Gelfand, Dr. Mike Simmers, and Andy Pryor, who all showed me the ropes (both in lab work and antics).

- Jon Park, for technical help and for keeping lab running smoothly when experiments got hectic.

- My classmates, for their constant camaraderie, shared adventures, stress relief, and advice over tea breaks. 
- My family and friends, whose encouragement was always a source of comfort, invigoration, and renewed optimism. I wouldn't have accomplished this without your support.

- My fiancé, Trevor Brown, for being my ultimate partner. Thank you for having such great faith in me, keeping me smiling, and having my back no matter the zip code or time zone. 


\section{TABLE OF CONTENTS}

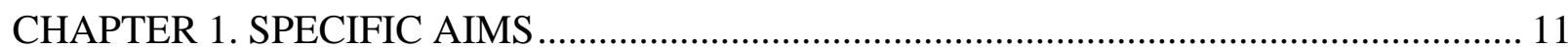

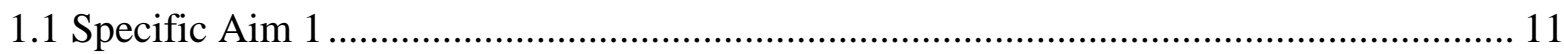

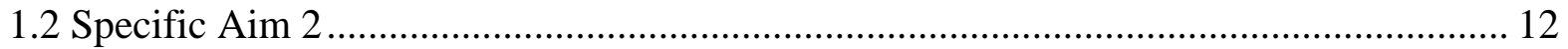

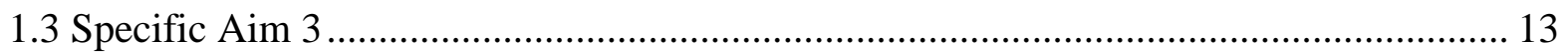

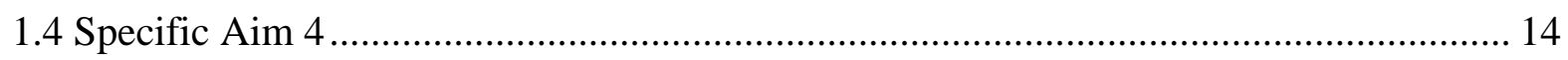

CHAPTER 2. BACKGROUND AND SIGNIFICANCE ………………………................. 15

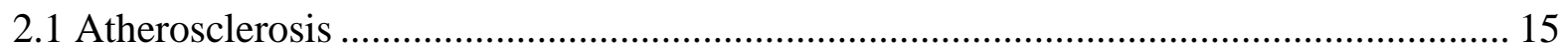

2.2 The Effects of Atheroprone Hemodynamics on Endothelial Function ............................. 16

2.3 PECAM in Shear Stress Mechanotransduction .............................................................. 18

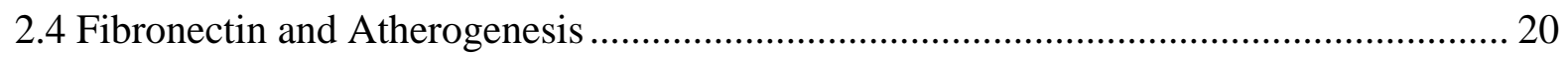

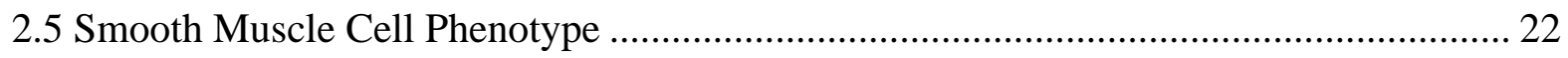

2.6 Secreted Protein Signaling ....................................................................................... 25

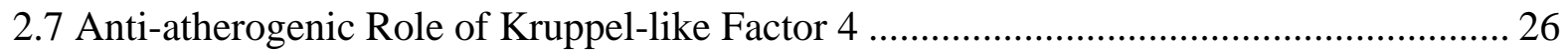

2.8 Central Hypotheses of the Dissertation ........................................................................ 27

CHAPTER 3. MATERIALS AND METHODS ………………………................................ 28

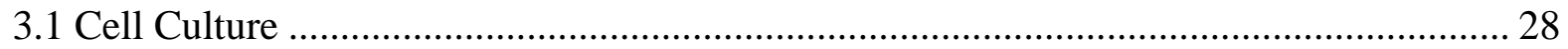

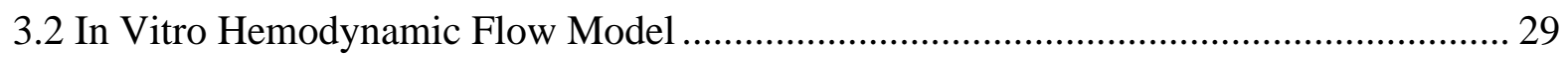

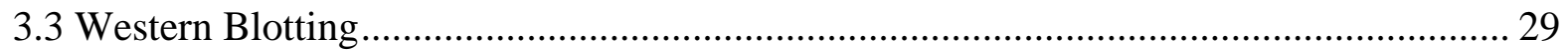

3.4 RNA Isolation and Real-Time RT-PCR Analysis............................................................ 30

3.5 siRNA Knockdown of PECAM, Fibronectin, and KLF4 ................................................. 31 
3.6 Viral Infection of Adenovirus NF-kB Luciferase Reporter and KLF4 Overexpression

Vector 32

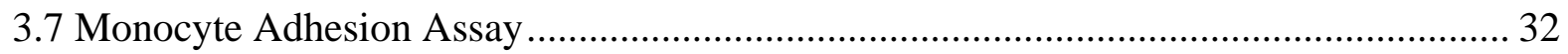

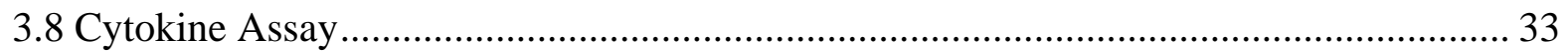

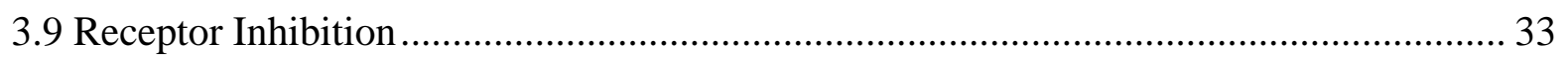

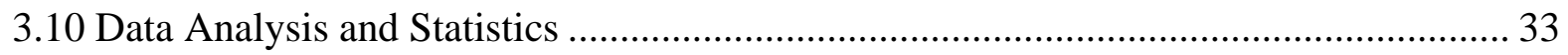

CHAPTER 4. ENDOTHELIAL PECAM REGULATES SMOOTH MUSCLE CELL

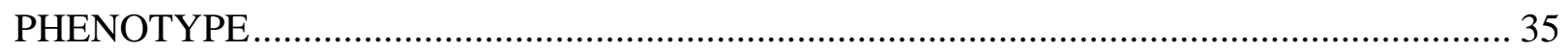

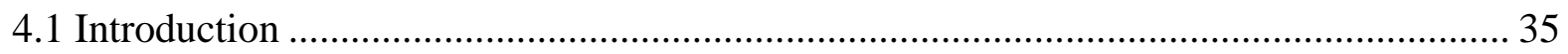

4.2 Hemodynamic Shear Stress Regulates EC and SMC Inflammatory Phenotypes ............ 36

4.3 Atheroprone Shear Stress Regulates SMC Inflammatory Phenotype via EC

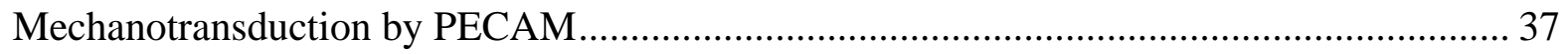

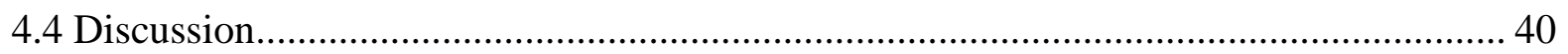

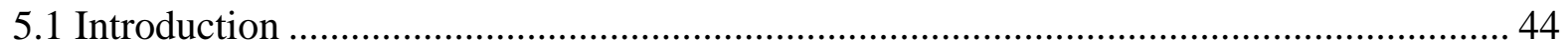

5.2 Endothelial FN Promotes SMC Inflammatory Phenotype …………………................... 45

5.3 SMC FN Promotes Positive Feedback of EC Inflammatory Phenotype ........................... 47

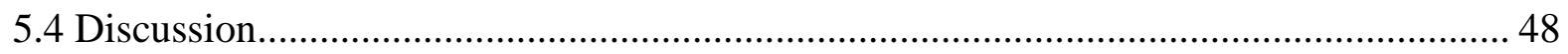

CHAPTER 6. SECRETED PROTEINS PROMOTE SMOOTH MUSCLE CELL

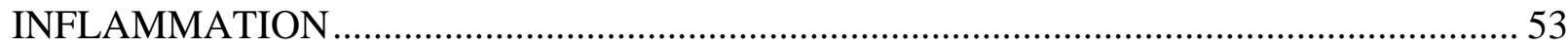

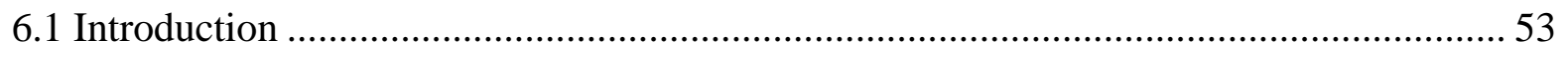

6.2 Endothelial Secreted Factors Regulate SMC Inflammatory Phenotype............................ 54

6.3 Endothelial Secreted Proteins are Modulated by FN ................................................... 56

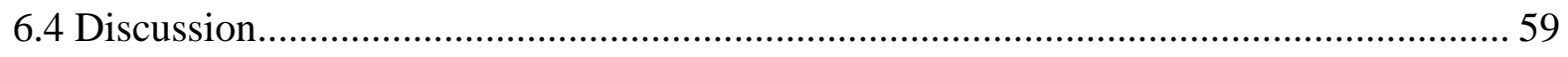


CHAPTER 7. KRUPPEL-LIKE FACTOR 4 ESTABLISHES AN ATHEROPROTECTIVE

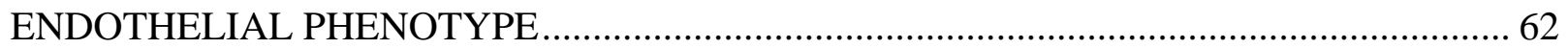

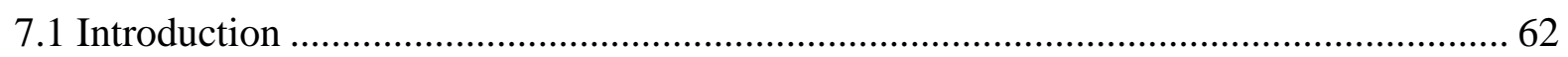

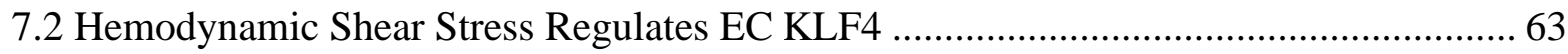

7.3 Overexpression of KLF4 Maintains an Anti-Inflammatory Phenotype ............................ 64

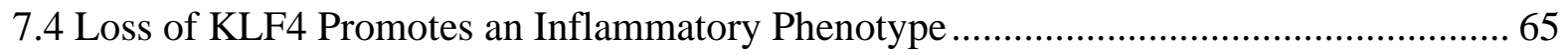

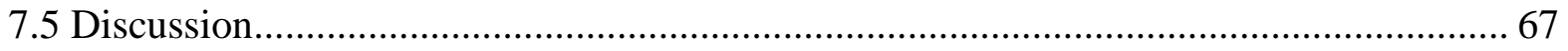

CHAPTER 8. SUMMARY, FUTURE DIRECTIONS, AND CLOSING REMARKS.............. 70

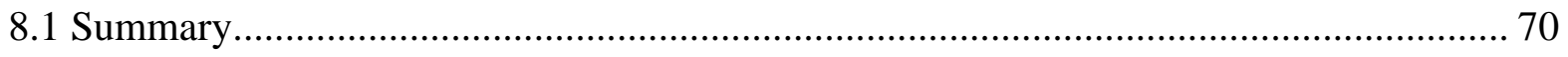

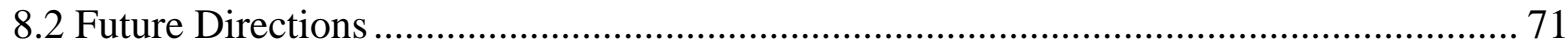

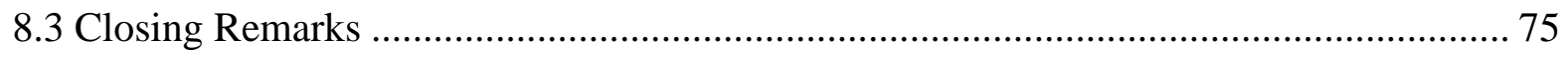

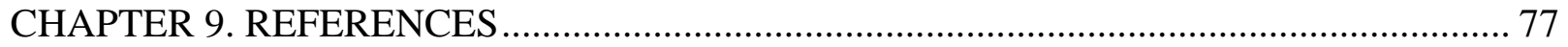




\section{CHAPTER 1. SPECIFIC AIMS}

\subsection{Specific Aim 1}

To determine the role of endothelial Platelet Endothelial Cell Adhesion Molecule-1 signaling in regulating smooth muscle cell atheroprone phenotype.

PECAM is a mechanosensory protein important for the initiation and progression of atherosclerosis. PECAM promotes inflammatory signaling pathways in ECs in response to atheroprone hemodynamic shear stress. Using a co-cultured EC/SMC system, our lab has shown that SMCs switch to an inflammatory phenotype when overlying ECs are exposed to atheroprone shear stress compared to atheroprotective. In order to determine the role of EC PECAM in signaling SMC inflammation, the co-culture model was exposed to either atheroprone or atheroprotective shear stress. PECAM protein was knocked down in ECs using an siRNA approach, and changes to the SMC phenotype were measured through gene expression, protein expression, and inflammatory NF- $\mathrm{B}$ activity.

The hypothesis of this Aim is:

\section{Atheroprone flow-induced inflammatory phenotype in SMCs is dependent on the expression and signaling of endothelial cell PECAM.}




\subsection{Specific Aim 2}

To determine the regulatory role of the matrix protein fibronectin in promoting endothelial and smooth muscle cell atheroprone phenotypes.

The matrix protein FN has been implicated in the development of atherosclerosis. FN has increased deposition in atheroprone regions in vivo, and promotes inflammatory signaling pathways in vitro. In Aim 1, we discovered that FN expression is upregulated in both ECs and SMCs under atheroprone hemodynamics. To study the role of FN deposition in promoting EC and SMC inflammation, the co-culture model was exposed to atheroprone hemodynamic shear stress. FN protein was knocked down in ECs using siRNA, and changes to the SMC phenotype were measured through gene expression, protein expression, and NF-kB activity. Functional changes to the SMC phenotype were measured by a monocyte adhesion assay. A rescue experiment with exogenous FN added to EC media was used to assess the ability for ECs to reincorporate FN into their matrix and resume inflammatory signaling. Finally, FN protein was knocked down in SMCs, and changes to the EC phenotype were measured to assess a potential positive-feedback signaling mechanism. EC protein markers and NF-kB activity were used to assess the level of EC inflammation.

The hypothesis of this Aim is:

FN expression in both ECs and SMCs acts as a positive regulator of inflammation. 


\subsection{Specific Aim 3}

To determine the role of secreted proteins in promoting smooth muscle cell atheroprone phenotype.

ECs and SMCs produce and respond to secreted proteins, making these short-range mediators a potential signaling mechanism between ECs and SMCs downstream of the PECAM/FN pathway. To study the proteins secreted in our co-culture system, cell media was collected from EC siPECAM and siFN atheroprone experiments. To determine whether differences in secreted proteins from control versus knockdown conditions would change the inflammatory response in SMCs, static SMCs were treated with conditioned media from these experiments. SMC NF- $\mathrm{kB}$ activity was used as a measure of the SMC inflammatory response. Next, a recovery experiment was performed to study the effect of conditioned media in the coculture system in real-time. ECs treated with siFN to knockdown FN expression were exposed to factors secreted from a non-siRNA control experiment. SMC NF-kB activity was then used to measure the restoration of the SMC inflammatory phenotype. To screen media for secreted cytokines differentially regulated by FN, a multiplexing approach was used. Of the cytokines measured, highly expressed cytokines were then tested individually for their ability to induce SMC NF- $\kappa$ B activity.

The hypothesis of this Aim is:

\section{Secreted proteins downstream of the PECAM/FN pathway modulate Atheroprone phenotypes.}




\subsection{Specific Aim 4}

To determine the role of endothelial Kruppel-like Factor 4 in establishing an atheroprotective endothelial phenotype.

KLF4 is a transcription factor with a suggested atheroprotective role in the endothelium. Overexpression of KLF4 in vitro induces anti-inflammatory and anti-thrombotic factors such as eNOS and thrombomodulin. The physiologic role of KLF4 in atherosclerosis has not been elucidated. To study the role of KLF4 in establishing an atheroprotective phenotype, monocultured ECs were exposed to either atheroprotective or atheroprone hemodynamic shear stress that mimics human physiology. EC gene and protein expression was used to measure the resulting EC phenotype. Next, the role of KLF4 overexpression was assessed through adenovirus containing KLF4 or empty vectors. ECs overexpressing KLF4 were subjected to atheroprone hemodynamics to test whether KLF4 could promote an anti-inflammatory EC phenotype despite the shear stress environment. Finally, ECs with KLF4 inhibited through siRNA were subjected to atheroprotective hemodynamics. EC gene expression was then used to measure whether loss of KLF4 would lead to an inflammatory EC phenotype despite the protective shear stress environment.

The hypothesis of this Aim is:

Atheroprotective-induced KLF4 expression reduces EC inflammation, and high levels of KLF4 promote an atheroprotective phenotype in atheroprone flow conditions. 


\section{CHAPTER 2. BACKGROUND AND SIGNIFICANCE}

\subsection{Atherosclerosis}

Atherosclerosis is a chronic inflammatory disease of the large and medium-sized arteries that represents a leading cause of death worldwide. It is characterized by the development of plaques in the blood vessel wall, leading to vessel thickening and potentially thrombosis (see reviews ${ }^{1,2}$ ). The arterial wall contains three layers: the tunica intima at the luminal interface is lined by a monolayer of ECs, the underlying tunica media contains SMCs surrounded by extracellular matrix, and the outermost adventitia contains mast cells and microvessels.

The earliest precursor of atherosclerosis is the "priming" of the endothelium towards an "activated" or pro-inflammatory state by regional shear stresses. "Priming" by region-specific blood flow patterns in combination with other factors including presence of modified lipoproteins, hypertension, and pro-inflammatory cytokines, can further "activate" the endothelium. "Activated" ECs express adhesion molecules that allow adherence of circulating leukocytes and secrete chemoattractant proteins that enhance the recruitment and migration of bound leukocytes into the intima. Together, these actions result in an accumulation of monocytes (the most prevalent inflammatory cell in plaques) in the intima where they differentiate into macrophages. The "activated" endothelium also has increased permeability to low-density lipoprotein (LDL). This is the first visible sign of atherosclerosis, termed the fatty streak. The progression of atherosclerosis from a fatty streak begins with the accumulation of LDL in the vessel wall, where if it is not removed, becomes biochemically modified/oxidized. Oxidized LDL further inflames the ECs and is endocytosed by resident macrophages. These lipid-laden macrophages (known as foam cells due to the appearance of their lipid vacuoles) 
attempt to remove pro-inflammatory oxidized LDL, however, this mechanism can become overwhelmed leading to foam cell apoptosis. Inefficient clearance of these dead cells results in the formation of a lipid-rich necrotic core in the developing plaque.

As the atherosclerotic lesion progresses, SMCs migrate from the media to the intima in an attempt to contain the growing lesion and necrotic core. In the intima, the SMCs proliferate in response to growth factors and secrete an extracellular matrix of collagen and elastin, forming a fibrous cap on the luminal side of the plaque. The composition of this cap is influenced by resident cells and is important in determining the vulnerability of the plaque to rupture. Inflammatory cells present in the lesion produce proteases that degrade the extracellular matrix and mediators that inhibit SMC matrix production. In contrast, calcification of the plaque by pericyte-like cells works to stabilize the lesion.

If the plaque remains stable, arterial wall thickening can reduce blood flow leading to tissue ischemia or angina. If the plaque is not stabilized, the fibrous cap may rupture exposing the contents of the necrotic core to circulating blood, which triggers blood coagulation. The resulting thrombus may obstruct blood flow locally or creates an embolism distally leading to heart attack or stroke. Since the outcome of both stable and unstable advanced lesions results in clinical manifestations, understanding the mechanisms during the initial development of atherosclerosis may provide an opportunity to address this global health problem therapeutically.

\subsection{The Effects of Atheroprone Hemodynamics on Endothelial Function}

The endothelium is a highly active and heterogenic structure that forms the cellular lining of blood vessels. In addition to providing a barrier between blood and the surrounding 
vessel wall, it regulates vasomotor tone ${ }^{3}$, trafficking of nutrients and oxygen ${ }^{4}$, and infiltration of blood leukocytes, particularly inflammatory cells ${ }^{5}$. In order to balance these diverse functions, the activation state of the endothelium is determined by the integration of many different stimuli. The endothelium interacts with circulating blood and inflammatory cells, soluble factors, extracellular matrix proteins in the vessel wall, and surrounding SMCs while being subjected to pressure, stretch, and shear stress ${ }^{6}$. Perturbations to a variety of these stimuli can disturb the homeostasis of the endothelium leading to activation and downstream atherosclerosis.

While atherosclerosis is associated with a variety of risk factors, including age, family history, obesity, and diet, this complex disease is found to develop preferentially in regions of disturbed hemodynamic shear stress ${ }^{7-9}$. Disturbed, or atheroprone, shear stress is found at vessel branch points and regions of high curvature characterized by low, oscillatory flow ${ }^{10,11}$. In contrast, high non-reversing flow is atheroprotective. The inflammatory response is tightly regulated by individual frequency harmonics contained within the atheroprone shear stress waveform, emphasizing the importance of using human-derived waveforms for the study of human atherogenesis ${ }^{12}$. Understanding the differences in molecular signaling caused by these flow patterns is key to understanding the initiation of atherosclerosis, and to identifying early prevention targets.

ECs are able to distinguish between atheroprotective and atheroprone shear stress signals. Atheroprotective shear stress induces EC alignment ${ }^{7}$ and activates anti-inflammatory and anti-thrombotic pathways through transcription of endothelial NOS (eNOS) ${ }^{9}$, Kruppel-like factor $2(\mathrm{KLF} 2)^{13,14}$, and NRF2-dependent anti-oxidative stress genes such as Hemooxygenase$1(\mathrm{HO}-1)^{15}$ and manganese superoxide dismutase (MN-SOD) ${ }^{16}$. Conversely, atheroprone shear 
stress induces inflammatory transcription factors such as nuclear factor kappa B (NF- $\mathrm{B})^{7}$, and T-cell specific transcription factor $(\mathrm{TCF})^{17}$. ECs exposed to atheroprone shear stress express increased monocyte adhesion molecules such as E-selectin ${ }^{18}$, vascular cell adhesion molecule-1 $(\mathrm{VCAM})^{19}$, and intercellular adhesion molecule-1 (ICAM) $)^{20}$, and secrete inflammatory cytokines involved in monocyte trafficking such as interleukin 8 (IL-8), interleukin 6 (IL-6) ${ }^{21}$, growth-related oncogene $\alpha(\mathrm{GRO} \alpha)^{22}$, monocyte chemoattractant protein-1 (MCP-1 $)^{23}$, vascular endothelial growth factor (VEGF) ${ }^{24}$, and bone morphogenic protein 4 (BMP-4) ${ }^{25}$. This results in a preferential accumulation of monocytes in atheroprone regions ${ }^{26-28}$.

Other endothelial functions are also perturbed under atheroprone shear stress, leading to an inflammatory EC phenotype. ECs under atheroprone shear stress show increased proliferation and do not align in the direction of flow ${ }^{29}$. Vasomotor activity is shifted towards SMC contraction due to a decrease in eNOS and increase in endothelin-1 (ET-1) compared to steady atheroprotective flow $^{30,31}$. Disturbed shear stress induces inflammation by increasing NADPH oxidase production of reactive oxygen species $^{32}$. Atheroprone flow leads to p21activated kinase (PAK) phosphorylation and recruitment to cell-cell junctions which elevates endothelial permeability in vitro ${ }^{33}$ and in mouse atheroprone regions in vivo ${ }^{34}$. The combination of these hemodynamic-induced changes results in an endothelium that is chronically inflamed and primed for disease development.

\subsection{PECAM in Shear Stress Mechanotransduction}

The endothelium responds to changes in fluid shear stress through the conversion of mechanical forces to biochemical signals (mechanotransduction) ${ }^{35}$. Mechanotransduction has been proposed to occur via integrins, the glycocalyx, G-proteins, caveolae, cytoskeletal and 
junctional proteins (see review ${ }^{36}$ ). Of particular interest is a mechanosensory complex ${ }^{37}$ located at cell-cell junctions composed of platelet endothelial cell adhesion molecule-1 (PECAM), vascular endothelial-cadherin (VE-cadherin), and vascular endothelial growth factor receptor 2 (VEGFR2), that activates downstream integrins ${ }^{38}$ and NF- $\mathrm{KB}^{39}$. PECAM is thought to be the "mechano-sensor" of the complex since it is tyrosine phosphorylated by direct tension applied through magnetic antibody-coated beads ${ }^{40}$, and is activated within seconds of force application $^{41,42}$.

PECAM is a transmembrane glycoprotein with six extracellular Ig folds and two intracellular immunoreceptor tyrosine inhibitory motifs (ITIM). When PECAM is phosphorylated by Src family kinases, its ITIM acts as a docking site for the Src homology 2 domain of SHP- $2^{43}, 44$. PECAM and SHP-2 activity are required for downstream extracellular signal-regulated kinase (ERK) activation under shear stress ${ }^{45}$, and are associated with the transactivation of VEGFR2 ${ }^{46}$. Tyrosine phosphorylation of PECAM also modulates the shear stress-induced phosphorylation of Akt, eNOS, and downstream nitric oxide (NO) production ${ }^{47}$ and is associated with increased NF- $\mathrm{KB}$ activity in atheroprone regions ${ }^{12,}$. PECAM is required for Src activation and downstream phosphatidylinositol-3-OH kinase (PI3K) phosphorylation, which triggers integrin $\alpha_{v} \beta_{3}$ activity and NF- $\kappa$ B activity ${ }^{37}$. PECAM-dependent PI3K activation also activates the small GTPase RhoA to facilitate cell alignment through cytoskeletal changes and growth of focal adhesions ${ }^{49}$. These results establish the role for the

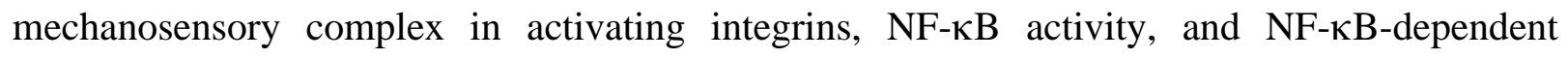
inflammatory genes involved in the development of atherosclerosis (ICAM, VCAM).

Mouse models of atherosclerosis have revealed PECAM's role in atherosclerotic lesion formation. Indeed, translocation of the NF- $\mathrm{KB}$ subunit, $\mathrm{p} 65$, is lost in atheroprone regions of 
PECAM $^{-/}$mice compared to their wild type (C57B6) controls ${ }^{37}$ illustrating the importance of PECAM in early "priming" events of the endothelium. Wild type mice (C57B6) do not develop atherosclerosis, but knocking out apolipoprotein-E $\left(\mathrm{ApoE}^{--}\right)$impairs lipid metabolism leading to the development of lesions in areas of disturbed shear stress similar to humans ${ }^{50}$. $\mathrm{ApoE}^{-/-}$ PECAM $^{--}$mice have reduced VCAM and ICAM expression, macrophage infiltration into the vessel wall, and atherosclerotic lesion size compared to $\mathrm{ApoE}^{-/}$in the lesser curvature of the aortic arch (an atheroprone region) ${ }^{51,52}$. In LDLR $^{-/-}$mice, PECAM $^{-/-}$had atheroprotective effects in the lesser curvature of the aortic arch, but atheroprone effects in the atheroprotective region of the descending aorta ${ }^{53}$. Therefore, the mechanical sensing of PECAM has both proand anti-atherogenic roles, but the pro-atherogenic role dominates in atheroprone hemodynamic regions.

\subsection{Fibronectin and Atherogenesis}

FN is a multifunctional extracellular matrix glycoprotein, which is usually present as a dimer of two subunits linked by disulfide bonds at their C-termini. Human cellular FN has up to 20 different variants, caused by alternative splicing of the exons in a single FN pre-mRNA ${ }^{54}$. In addition to many FN-FN binding sites for fibrillogenesis, $\mathrm{FN}$ is a ligand for at least a dozen integrins, heparin, fibrin, and collagen (see review ${ }^{55}$ ). This diverse array of differentially regulated binding capabilities drives FN involvement in tissue formation, maintenance, and remodeling through the promotion of cell adhesion, motility, matrix formation, and cytoskeletal organization ${ }^{56}$. It is widely expressed and necessary for vertebrate development, with $\mathrm{FN}^{-1-}$ mice showing early embryonic lethality ${ }^{57}$. It is also important to vascular remodeling, where $\mathrm{FN}$ is an important regulator of carotid intima and media thickening and SMC phenotypic modulation ${ }^{58}$. 
Vascular SMCs strongly adhere to FN-coated beads in an integrin-dependent manner, forming focal adhesions and generating myogenic forces, while collagen type I, laminin, and vitronectin matrix proteins fail to induce a response ${ }^{59}$.

FN is of particular interest because recent evidence has shown preferential FN deposition in the sub-endothelial layer in pre-atherosclerotic and mature atherosclerotic lesions in humans ${ }^{60-63}$ and animals ${ }^{64,65}$. Pre-atherosclerotic FN is found in both atherosclerosis-resistant C57/B6 mice on a normal chow diet and in hypercholesterolemic $\mathrm{ApoE}^{-/-}$mice on a high fat Western diet. In the SMC-containing neointima and media layers, a FN rich matrix is observed in atheroprone regions of $\mathrm{ApoE}^{-/-}$mice, compared to atheroresistant regions ${ }^{66}$. Likewise, FN is present in the neointima and media of the lesser curvature and carotid bifurcation (atheroprone region) in aortas of C57/B6 mice, as opposed to the greater curvature and common carotid $\operatorname{artery}^{66}$.

In addition to focal deposition in atheroprone regions, $\mathrm{FN}$ has also been linked to inflammatory pathways in vitro. Orr et al. found that ECs plated on FN are capable of regulating the pro-inflammatory transcription factor NF-אB, while ECs plated on collagen or laminin are not ${ }^{64}$. This response is dependent on the activation of p21-activated kinase (PAK) in response to shear stress for ECs plated on FN, but not on collagen ${ }^{33}$. PAK also regulates endothelial permeability in a FN-dependent manner ${ }^{67}$. The focal deposition of FN was found to depend on the hemodynamic environment. FN deposition is induced by atheroprone flow but not atheroprotective flow in vitro. FN deposition promotes NF- $\mathrm{BB}$ activation and creates a positive feedback loop that maintains inflammation ${ }^{48}$. This FN deposition by ECs is dependent on flow sensing by PECAM in vivo and in vitro ${ }^{48}$. Therefore, the mechanotransduction of 
atheroprone shear stresses via PECAM promotes atherosclerosis in part by regulating focal deposition of $\mathrm{FN}$ and activation of $\mathrm{NF}-\kappa \mathrm{B}$.

\subsection{Smooth Muscle Cell Phenotype}

During the development of an atherosclerotic lesion, SMCs undergo vast phenotype changes. These changes have been classically reduced to a "phenotypic switch" from a normal, contractile phenotype to a proliferative, synthetic one. This switch is a response to changes in the SMC's local environment such as mechanical forces, growth factors, and interactions with

extracellular matrix and with other cells (see reviews ${ }^{68,}{ }^{69}$ ). In their contractile, quiescent phenotype, vascular SMCs regulate blood vessel tone with low proliferation, expression of contractile proteins, calcium regulatory proteins, and ion channels required for force generation. The differentiated contractile phenotype is specifically marked by smooth muscle $\alpha$-actin (SM $\alpha \mathrm{A})$, smooth muscle myosin heavy chain (SM MHC), and myocardin. In response to vascular injury, SMCs switch to a synthetic phenotype that is highly proliferative and migratory in order to aid in vascular repair. The synthetic phenotype may show increased proliferation markers (e.g. Cyclins), decreased SM $\alpha$ A and SM MHC, rounding of the cell from its usual elongated morphology, and changes to its calcium handling. However, this two-state model is too binary to adequately explain the variety of phenotypes displayed by the SMC.

In atherosclerosis, SMCs display an inflammatory phenotype rather than a simply contractile or synthetic phenotype. The inflammatory SMC phenotype is characterized by a decrease in markers of contractility, an increase in proliferation and migration, and expression of inflammatory genes ${ }^{70}$. Inflammatory NF- $\mathrm{B}$ activity in SMCs is observed in atherosclerotic lesions in vivo ${ }^{71}$, and VCAM is expressed in SMCs in both the intima and media of regions of 
intimal thickening ${ }^{72}$. SMC expression of VCAM has also been associated with nuclear factor of activated T cells (NFAT) transcriptional activation ${ }^{73}$, which is known to induce proliferation and cell migration ${ }^{74,75}$. JAK-STAT signaling may also be involved in the inflammatory SMC phenotype due to its increased expression after vascular injury ${ }^{76}$ and necessity during angiotensin II stimulated proliferation and migration ${ }^{77,}$. ${ }^{78}$ In response to pro-inflammatory cytokines found in atherosclerotic lesions, SMCs increase their own expression of MCP-1 which promotes recruitment of monocytes to the growing plaque ${ }^{79}$. Also in response to atheroprone conditions, SMCs increase expression of matrix proteins, such as $\mathrm{FN}^{80}$ (which transiently activates NF- $\mathrm{KB}$ activity ${ }^{81}$ ) and collagen I (which reduces SMC differentiation in its monomeric form ${ }^{73}, 82$ ). While this phenotype may be advantageous in advanced atherosclerotic lesions due to its promotion of stable fibrous caps, it works to further inflame the vessel wall during the early stages of atherosclerosis. The switch from a contractile to an inflammatory phenotype plays an important role in the initiation of atherosclerosis, and much remains unknown about the environmental cues that control it.

Previous studies have investigated the communication between ECs and SMCs using co-culture models, either under static conditions or in parallel plate flow chambers that provide a constant shear stress. Chiu et al. demonstrated an anti-inflammatory effect of SMC on EC under shear stress. mRNA expression of ICAM, VCAM, and E-selectin in static ECs was increased when co-cultured with SMCs, but decreased when the co-culture was subjected to laminar shear stress ${ }^{83}$. The same group found a similar anti-inflammatory role for laminar shear stress in the co-culture system through the inhibition of NF-KB activation ${ }^{84}$, and through the activation of PPAR-alpha/delta to induce a contractile SMC phenotype ${ }^{85}$. Truskey et al. have used EC/SMC co-culture in the area of tissue-engineering, and showed that cells in co-culture 
express less thrombogenic tissue factor than in monoculture and that SMCs express more contractile calponin in co-culture ${ }^{86}$. ECs receive anti-inflammatory feedback when in direct contact with SMCs, with decreased adhesion molecules and increased KLF2 expression under flow or TNF- $\alpha$ treatment compared to monoculture ${ }^{87}$. This evidence points to the value of coculture studies in understanding both EC to SMC and SMC to EC signaling mechanisms.

While valuable information has been obtained from these co-culture models, the difference between atheroprone and atheroprotective flow patterns may be more relevant to the development of atherosclerosis than that of static and steady flow patterns ${ }^{7,8}$. In order to study the influence of EC signaling over SMC phenotype in atherosclerosis, our group developed a novel co-culture model ${ }^{88}$, which applies human-derived shear stress patterns to ECs that are cultured above a layer of SMCs (Figure 1). A porous membrane separates the two cell types, allowing for signaling cross-talk, as well as molecular manipulations to either cell type. Signaling between ECs and SMCs is difficult to study in intact arteries, making this model especially useful in determining how cross-talk modulates the SMC phenotype. Our model has successfully shown that shear stress applied only to the ECs alters SMC phenotype in agreement with phenotypes classically defined in atherosclerosis in human arteries ${ }^{88}$. Similar to in vivo vessel physiology, ECs align in the direction of flow, whereas SMCs align perpendicular to flow. Inflammatory VCAM expression (and other pro-inflammatory proteins) is increased in both ECs and SMCs in response to atheroprone flow. Additionally, our model was used to demonstrate a role for the cytokine interleukin- 8 to limit VCAM expression, thereby limiting further inflammation via EC SMC cross communication ${ }^{72}$. 

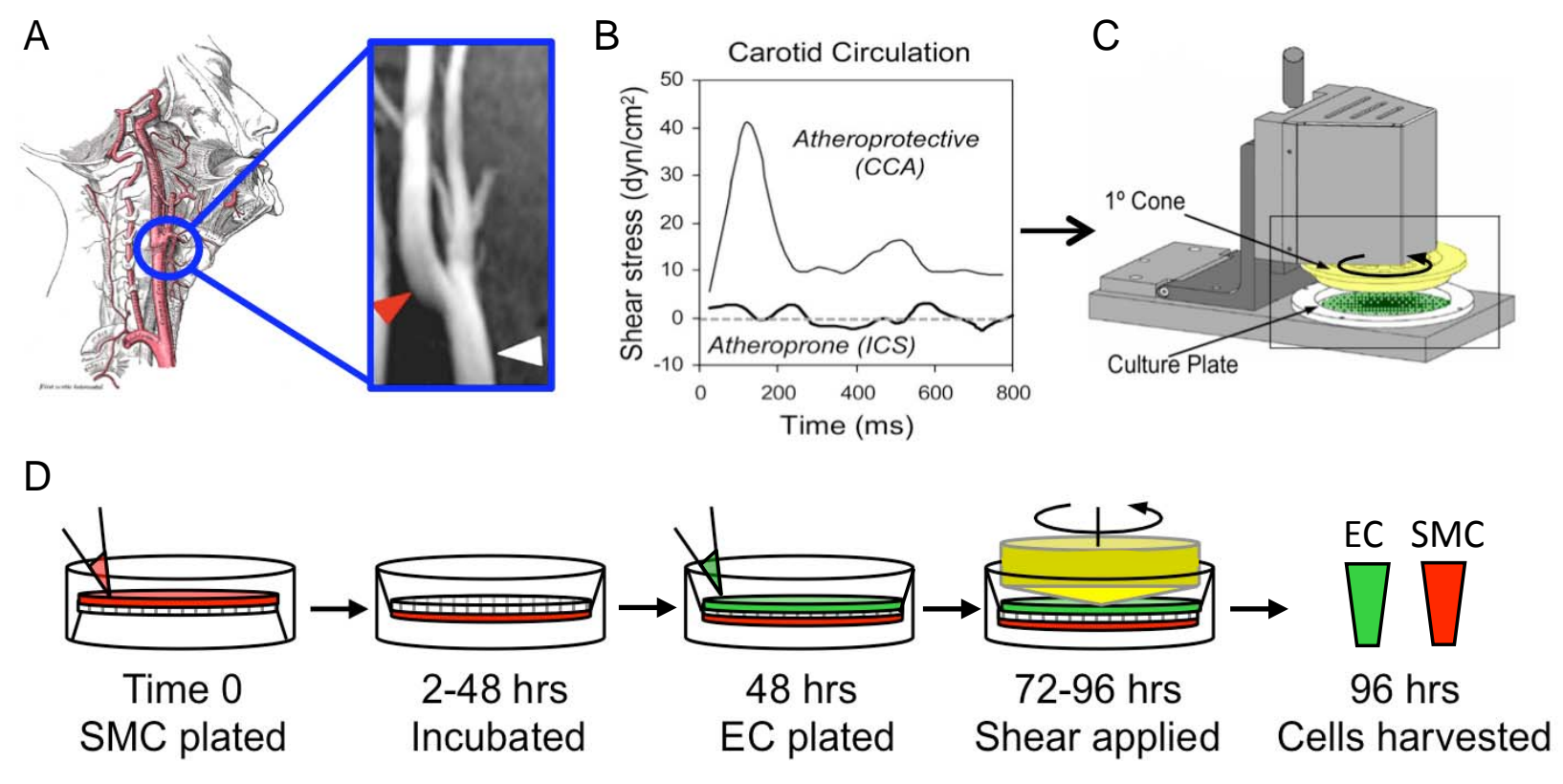

Figure 1. Measurement and recapitulation of atheroprotective and atheroprone blood flow in human carotid arteries. (A) Blood flow was measured using MRI in the internal carotid sinus (ICS, red arrow) and the common carotid artery (CCA, white arrow). (B) Shear stress waveforms for atheroprotective and atheroprone flow over time, as applied to the EC surface. (C) Schematic of the cone and plate flow device. (D) Plating timeline and schematic of cone applying shear stress to co-culture plate.

\subsection{Secreted Protein Signaling}

ECs and SMCs are able to produce and respond to secreted proteins (e.g., cytokines, chemokines, growth factors), making these short-range mediators a potential signaling mechanism between the two cell types. While ECs may influence inflammation in underlying SMCs through various mechanisms such as cadherins ${ }^{89}$, connexins $^{90}$, gases ${ }^{91}$, microRNAs ${ }^{92}$ and matrix proteins $s^{93}$, secreted proteins are of particular interest because a variety of proinflammatory cytokines have been found in atherosclerotic plaques ${ }^{94}$. Cytokines can be grouped into several classes, including interleukins (IL), colony-stimulating factors (CSF), interferons (IFN), growth factors (GF), and chemokines. They affect a full range of biological 
process spanning from embryonic development to immunology to cancer ${ }^{21}$, but are especially important in regulating inflammatory responses.

Secreted proteins have been implicated in both the response to shear stress and the phenotype modulation of SMCs. Shear stress is known to differentially regulate EC secretion of IL-1, IL- ${ }^{95}$, and IL- $8^{96,97}$. Low shear stress upregulates MCP-1, GRO- $\alpha$ and fractalkine in an in vivo mouse model ${ }^{98}$, and in in vitro flow models ${ }^{22,99}$. Aortic SMCs treated with MCP-1 in vitro have a less differentiated phenotype with decreased $\mathrm{SM} \alpha \mathrm{A}^{100}$. Shear stress also induces EC secretion of IL-1 and PDGF-BB to stimulate SMC migration in vitro via MAPK pathways $^{101}$. The PDGF isoform AA stimulates arterial SMCs proliferation in vitro, through enhanced phosphorylation of ERK1/2 and $\mathrm{Akt}^{102}$. These results provide potential mechanisms for EC to SMC signaling in response to shear stress.

\subsection{Anti-atherogenic Role of Kruppel-like Factor 4}

In contrast to the inflammatory NF- $\mathrm{B}$ pathway through PECAM, anti-atherogenic signals can be initiated by atheroprotective shear stress in an effort to maintain vessel homeostasis. One factor of interest is the zinc-finger family transcription factor Kruppel-like factor 4 (KLF4). KLF4 is expressed in endothelial cells in vitro and in vivo ${ }^{103}$, and is induced by laminar shear stress ${ }^{104}$. It promotes quiescence in EC by inducing eNOS and thrombomodulin (TM), and by repressing the p65-mediated activation of $\mathrm{NF}-\mathrm{\kappa B}$ and expression of downstream VCAM and plasminogen activator inhibitor (PAI-1). KLF4 also has the functional effect of decreasing monocyte adhesion to TNF $\alpha$-activated ECs ${ }^{103}$. When the anti-inflammatory KLF2 is inhibited in a $\mathrm{KLF}^{+/-} \mathrm{ApoE}^{-/-}$mouse, KLF4 mRNA expression increases, potentially as a compensation mechanism to prevent inflammation ${ }^{13}$. These 
observations suggest an atheroprotective role for KLF4, which we can test using our physiologic shear stress patterns. We hypothesize flow-induced KLF4 expression reduces EC inflammation, and that high levels of KLF4 promote an atheroprotective phenotype under atheroprone flow conditions.

\subsection{Central Hypotheses of the Dissertation}

The activation of an inflammatory EC phenotype by hemodynamic shear stress is crucial to the development of atherosclerosis. While shear stress is known to regulate EC signaling, the influence of that signaling on the SMC phenotype is largely unknown. The overall goals of this dissertation are to 1) investigate the role of hemodynamic regulated EC signaling pathways in modulating the SMC inflammatory phenotype and to 2) test the ability of KLF4 to inhibit EC inflammation. The central hypotheses of this work are:

\section{We hypothesize that mechanical sensing by the PECAM/FN pathway in endothelial cells regulates the smooth muscle cell inflammatory phenotype. \\ 2. We hypothesize that KLF4 promotes an atheroprotective endothelial phenotype.}

These hypotheses will provide the following specific advancements to the field:

- A new appreciation for the ability of shear stress to drive underlying SMC phenotype, and for that SMC phenotype to further advance inflammation in the atheroprone environment

- Evidence that KLF4 is a potent inhibitor of shear stress driven inflammation in ECs 


\section{CHAPTER 3. MATERIALS AND METHODS}

\subsection{Cell Culture}

Primary human ECs were purchased from Lonza, Vec Technologies, Cell Applications Inc., or isolated from umbilical cord veins (HUVEC) and expanded as previously described ${ }^{105}$. Tissue procurement was approved by the Human Investigation Committee of the Martha Jefferson Hospital System (IRB Approval \#0179). ECs were cultured and passaged in M199 media supplemented with 10\% fetal bovine serum (FBS; Hyclone), 2mM L-glutamine (Gibco), $5 \mu \mathrm{g} / \mathrm{ml}$ endothelial cell growth supplement (ECGS; Biomedical Technologies), $10 \mu \mathrm{g} / \mathrm{ml}$ heparin (Sigma), and $100 \mathrm{U} / \mathrm{ml}$ penicillin-streptomycin (Invitrogen), and were used between passage 2 and 3 .

Human umbilical vein SMCs (Cell Applications, Inc) were grown and passaged in smooth muscle cell growth medium (Cell Applications, Inc). SMCs were plated onto a $1 \%$ gelatin-treated membrane of a 75-mm-diameter transwell co-culture dish (Corning), which was inverted, at a density of 40,000 cells $/ \mathrm{cm}^{2}$ for 2 hours. The transwell was then placed back into its supporting dish with low serum media (M199 supplemented with 2\% FBS, 2mM Lglutamine, and $100 \mathrm{U} / \mathrm{ml}$ penicillin-streptomycin) for 48 hours. ECs were then plated on the top surface of the gelatin-treated transwell membrane at a density of 80,000 cells $/ \mathrm{cm}^{2}$ in low serum media for 24 hours.

The Blackman lab has validated that experiments with human umbilical vein ECs and SMCs produce similar results to those with human aortic EC and SMCs. Therefore due to high cost of purchasing aortic endothelial cells and our publication history using this model ${ }^{72,88,106}$, 
we choose to continue to use EC and SMC from human umbilical veins as a valid and proven model.

\subsection{In Vitro Hemodynamic Flow Model}

Our lab developed a novel co-culture in vitro model where arterial flow patterns measured directly from the human circulation with high resolution MRI are applied to human $\mathrm{ECs}^{88}$. Flow profiles from the human common carotid artery (CCA) and internal carotid sinus (ICS) provide atheroprotective and atheroprone shear stress patterns, respectively (Figure 1). Regional shear stress patterns (either atheroprone or atheroprotective) are applied to the EClayer in a pulsatile manner with a flow device consisting of a cone-and-plate viscometer driven by a controllable motor (Figure 1C). The flow device provides continuous media exchange to both cell layers, maintains physiological temperatures $\left(37^{\circ} \mathrm{C}\right)$, and controls humidity and $\mathrm{CO}_{2}$. Cell media for flow experiments has low serum (2\% FBS) and 4\% Dextran (Sigma) by weight to increase viscosity to a physiologic level of 4cP. In experiments with exogenous FN, EC flow media was supplemented with $10 \mu \mathrm{g} / \mathrm{mL} \mathrm{FN}$ for the last 8 hours of the experiment (Invitrogen). All experiments are conducted for 24 hours.

For Aim 4, a similar monoculture in vitro model is used. The same shear stress patterns are applied to a confluent monolayer of ECs plated in a flat $10 \mathrm{~cm}$ dish as previously described ${ }^{105}$. Media, temperature, humidity and $\mathrm{CO}_{2}$ are controlled as above.

\subsection{Western Blotting}

Protein samples were lysed directly on the membrane or culture dish in MAPK sample buffer with 50mM DTT. After gel electrophoresis, samples were transferred to a nitrocellulose 
membrane using an iBlot system (Invitrogen). Western blotting is performed using the LiCor Odyssey system to measure relative FN (BD, 1:1000), VCAM (Santa Cruz, 1:500), SM $\alpha$ A (Sigma, 1:1000) and PECAM (Santa Cruz, 1:750) protein normalized to $\alpha$-tubulin (Sigma, 1:1000) to control for loading.

\subsection{RNA Isolation and Real-Time RT-PCR Analysis}

On completion of flow experiments, cells were immediately collected in cold DPBS. Total RNA was extracted using the PureLink Kit (Life Technologies) and reverse transcribed to cDNA using the iScript cDNA Synthesis kit (Bio-Rad). Primer sequences for genes studied are shown in Table 1. Genes were assayed in duplicate using real-time reverse transcriptase polymerase chain reaction (RT-PCR) with SYBR Green (Bio-Rad). Gene expression levels were normalized to the control gene $\beta$-2-microglobulin (B2M).

\begin{tabular}{|l|l|l|}
\hline Gene Name & Sense Primer & Anti-sense Primer \\
\hline $\begin{array}{l}\beta 2- \\
\text { microglobulin }\end{array}$ & AGCATTCGGGCCGAGATGTCT & CTGCTGGATGACGTGAGTAAACCT \\
\hline COX-2 & CTGCTCAACACCGGAATTTT & GAGAAGGCTTCCCAGCTTTT \\
\hline eNOS & CTCCATTAAGAGGAGCGGCTC & CTAAGCTGGTAGGTGCCTGTG \\
\hline E-Selectin & AATCCCAGTTTGTGAAGCTTTCCA & GCCAGAAGCACTAGGAAGACAATT \\
\hline Fibronectin & AACGATCAGGACACAAGGAC & CCTCTCACACTTCCACTCTC \\
\hline KLF2 & CACCGACGACGACCTCAACA & CGCACAGATGGCACTGGAAT \\
\hline KLF4 & GGCCAGAATTGGACCCGGTGTAC & GCTGCCTTTGCTGACGCTGATGA \\
\hline PAI-1 & GAGGTGCCTCTCTCTGCCCTCACCAACATT & AGCCTGAAACTGTCTGAACATGTCG \\
\hline SMaA & CACTGTCAGGAATCCTGTGA & CAAAGCCGGCCTTACAGA \\
\hline TM & CCTCCATGCATCTCATAGCA & CGGGTTGTGTGTCTGTTCAC \\
\hline VCAM & GTTTGTCAGGCTAAGTTACATATTGATGA & GGGCAACATTGACATAAAGTGTTT \\
\hline
\end{tabular}

Table 1. Gene primer sequences. 


\section{5 siRNA Knockdown of PECAM, Fibronectin, and KLF4}

For knockdown of PECAM, KLF4, and SMC FN, cells were plated at $60 \%$ confluence and transfected using $0.87 \mathrm{pmol}$ siRNA for scrambled oligonucleotides (D-001810, Thermo Scientific), human PECAM (L-017029-00, Thermo Scientific) or human KLF4 (L-005089-00) and 52uL of Oligofectamine (Invitrogen) in $6.9 \mathrm{~mL}$ of OptiMEM-1 (Invitrogen) for 5 hours. For knockdown of FN in ECs, cells were plated at $60 \%$ confluence and transfected with $1.2 \mathrm{pmol}$ siRNA for scrambled oligonucleotides or human FN (L-009853-00, Thermo Scientific) and $52 \mathrm{uL}$ of Oligofectamine (Invitrogen) in $6.9 \mathrm{~mL}$ of OptiMEM-1 (Invitrogen) for 5 hours. Each siRNA is a SMARTpool consisting of 4 transcripts mixed together to increase efficiency and reduce off-target effects. After 5 hours, cells were flooded with $8 \mathrm{~mL}$ of M199 growth media with 10\% FBS. For cells used in co-culture experiments, transfection was performed 24 hours before re-plating cells onto the transwell as demonstrated below (Figure 2).

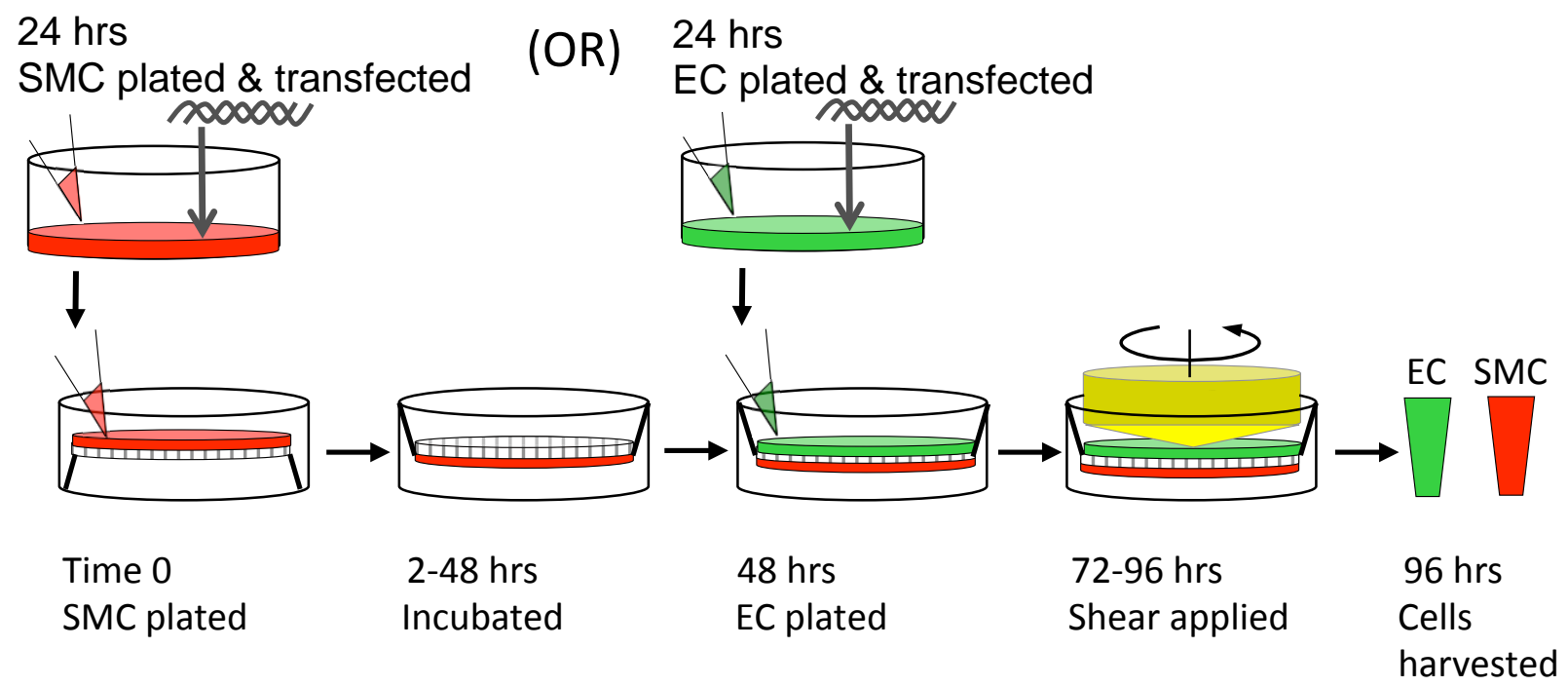

Figure 2. Plating timeline for siRNA transfection with co-culture. For experiments with SMC siRNA, cells were plated and transfected 24 hours before Time 0. For separate experiments with EC siRNA, cells were plated and transfected 24 hours before re-plating into co-culture. 


\subsection{Viral Infection of Adenovirus NF-kB Luciferase Reporter and KLF4 Overexpression Vector}

ECs and SMCs were infected with $50 \mathrm{MOI}$ adenovirus containing NF-kB-luciferase reporter (Vector Biolabs), 24 hours before exposure to shear stress. On completion of flow experiments, cells were immediately collected in cold DPBS, pelleted, and lysed with 100uL of Passive Lysis Buffer (Promega). Cells were passively lysed for 30 minutes at room temperature on a rotator, then assayed with Luciferase Assay Reagent (Promega). RLU's were normalized to protein concentration using a Coomassie Plus (Thermo Scientific).

For viral overexpression experiments, HUVEC were plated at 40,000 cells $/ \mathrm{cm}^{2}$ and infected with 10 MOI adenovirus containing KLF4 or Empty vectors. After 24 hours of infection, the adenovirus medium was exchanged with fresh medium. Flow experiments were started 48 hours after infection.

\subsection{Monocyte Adhesion Assay}

THP-1 monocyte cells were washed two times in DPBS and re-suspended in RPMI media (Gibco) at a concentration of $1 \mathrm{E} 6$ cells $/ \mathrm{mL}$. THP-1 cells were then labeled with $1 \mathrm{ug} / \mathrm{mL}$ Calcein AM (Life Technologies) at $37^{\circ} \mathrm{C}$ for 20 minutes before the labeling reaction was stopped with additional RPMI. Next, THP-1 cells were washed and re-suspended in M199. On completion of flow experiment, media was removed and the transwell was inverted in its dish. 2.2 million THP-1 cells in $2 \mathrm{~mL}$ M199 were then added on top of the SMC layer and incubated at $37^{\circ} \mathrm{C}$ for 20 minutes. After incubation, the transwell was re-inverted before washing two times in cold DPBS and fixing with $4 \%$ paraformaldehyde for 45 minutes. The transwell membrane was then washed four times in DPBS and mounted with DAPI mounting media (Life Technologies) between two coverslips. Membranes were imaged with a fluorescence 
microscope and the number of adhered monocytes was quantified using the ImageJ Particle Analyzer (NIH). Number of adhered monocytes was the average count from 10 images taken at different locations on the membrane.

\subsection{Cytokine Assay}

Flow experiment media was collected on completion of the flow experiment and stored at $-80^{\circ} \mathrm{C}$ before analysis. Cytokines were quantified using a MILLIPEX MAP human cytokine magnetic bead panel (HCYTOMAG-60K, EMD Millipore) as described by the manufacturer on a MAGPIX system (Luminex). Amount of secreted cytokine was calculated by multiplying the measured concentration by the total volume of media collected.

\subsection{Receptor Inhibition}

The selective inhibitor SB 225002 (Cayman Chemical) was used to inhibit the GRO- $\alpha$ receptor CXCR2. Static SMCs were plated in media with $2 \%$ FBS for 24 hours, then serumstarved during Ad-NF- $\kappa$ B-luciferase treatment for 24 hours to reach quiescence. Then, SMCs were pre-treated with 50nM SB 225002 or DMSO vehicle control for 2 hours before treatment with control or pre-conditioned siControl atheroprone media containing the same concentration of SB 225002 or DMSO for 24 hours. On completion of pre-conditioned media treatment, cells were processed for NF- $\kappa \mathrm{B}$ activity as above in Chapter 3.6.

\subsection{Data Analysis and Statistics}

For data normalized to a fold change of 1, statistical significance was determined through a 1 or 2 -tailed one sample $t$-test, testing the null hypothesis of the mean ratio equal to 
1. For non-normalized data, statistical significance was determined through a 1-tailed paired $t$ test. All statistics are presented as means $\pm \mathrm{SE}$, with significance at $P<0.05$. 


\section{CHAPTER 4. ENDOTHELIAL PECAM REGULATES SMOOTH MUSCLE CELL PHENOTYPE}

\subsection{Introduction}

Our lab has established that the pathway downstream of EC PECAM is important for the initiation and progression of atherosclerosis. PECAM is a mechanosensory protein that contributes to lesion formation by regulating the inflammatory transcription factor NF-kB. PECAM promotes atheroprone VCAM expression in vitro and in vivo, macrophage infiltration into the vessel wall, and increased atherosclerotic lesion size ${ }^{51,52}$. It also promotes FN deposition by ECs, which further increases NF- $\mathrm{KB}$ activity, leading to a sustained inflammatory phenotype $^{48}$. Using a co-cultured EC/SMC model, our lab has shown that SMCs switch to an inflammatory phenotype when over lying ECs are exposed to atheroprone flow compared to atheroprotective. This inflammatory SMC phenotype is marked by increased VCAM and decreased SM $\alpha$ A expression. Since SMC phenotype is dependent on the EC flow environment, and EC detect their flow environment by mechanotransduction through PECAM, we hypothesize that EC PECAM regulates SMC phenotype.

The goal of the present study was to determine if the atheroprone flow-induced inflammatory phenotype in SMCs is dependent on the expression and signaling of endothelial PECAM. Using siRNA to knock down PECAM in ECs, co-cultured SMCs were tested for a decrease in their inflammatory phenotype in response to atheroprone flow. SMC inflammation was assessed with expression of VCAM, COX-2, FN, and NF- $\mathrm{KB}$ activity. Understanding the mechanism by which the PECAM signaling axis affects SMC phenotype is important in the 
regional development of atherosclerosis and may provide novel ways to therapeutically target the endothelium to control the underlying SMC behavior.

\subsection{Hemodynamic Shear Stress Regulates EC and SMC Inflammatory Phenotypes}

We first sought to confirm that hemodynamic shear stress regulates both EC and SMC inflammatory phenotypes as in previous work ${ }^{88}$. Figure 3A shows pro-inflammatory EC phenotypes in response to atheroprone flow including decreased eNOS, KLF2, and KLF4. Atheroprone flow also increases EC inflammatory markers, such as E-Selectin and VCAM transcripts as well as FN deposition (Figure 3C), in agreement with mono-culture results ${ }^{48}$. As expected, this inflammatory phenotype is signaled to the underlying SMCs, which display increased VCAM and decreased SM $\alpha$ A expression (Figure 3B), indicating an inflamed and less-contractile phenotype. Interestingly, FN (which has not been previously explored in any co-culture system) showed a significantly increased SMC expression in response to atheroprone flow compared to atheroprotective flow similar to ECs (Figure 3B). Most strikingly, SMC NF- $\mathrm{KB}$ activity was significantly higher in atheroprone flow compared to atheroprotective (Figure 3D). 
A

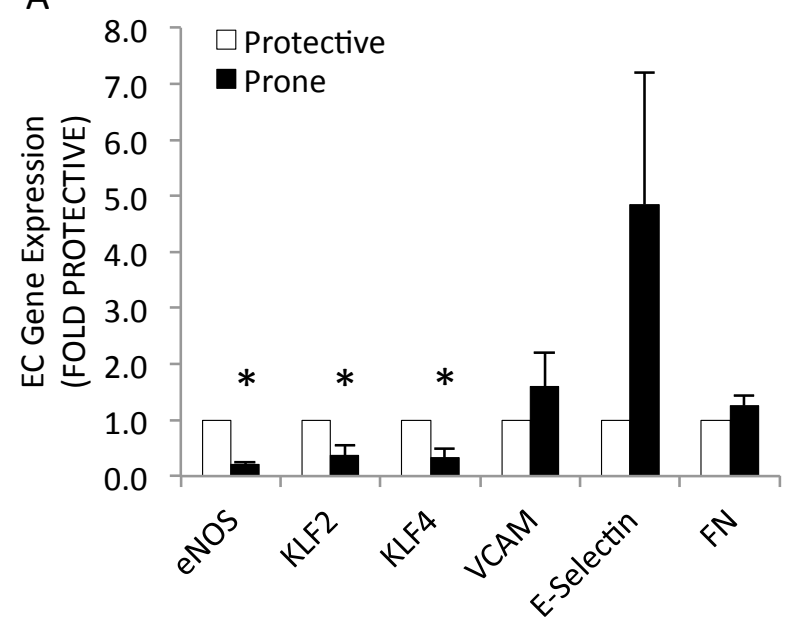

C

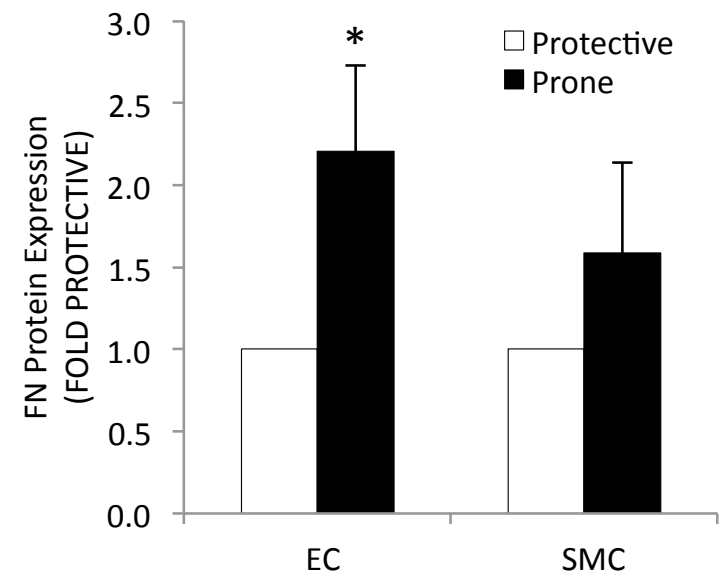

B

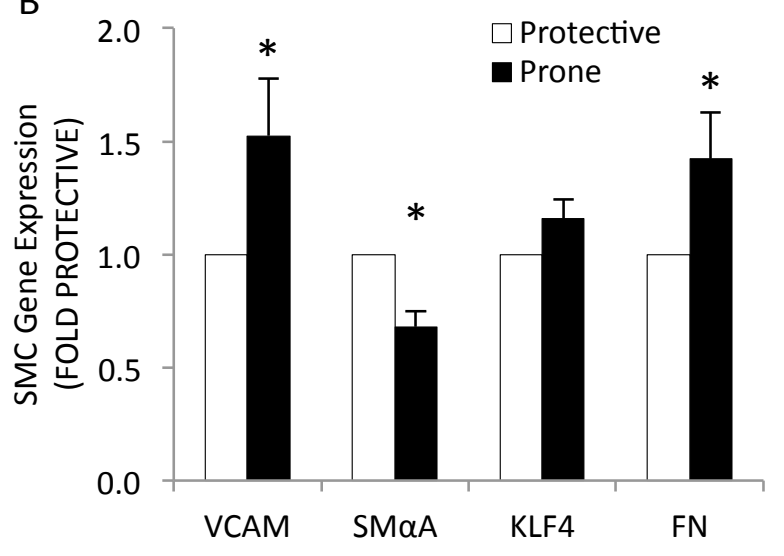

D

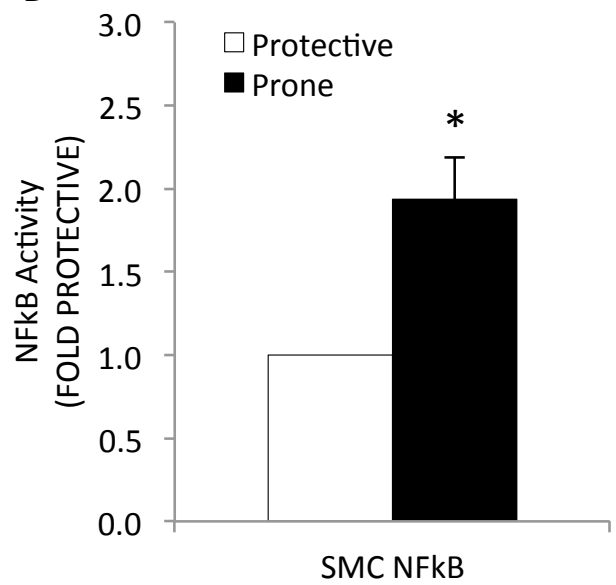

Figure 3. The hemodynamic environment regulates EC and SMC inflammatory phenotypes. (A) EC gene expression under atheroprone vs atheroprotective flow (n=6-10). (B) SMC gene expression under atheroprone vs atheroprotective flow $(\mathrm{n}=10)$. (C) EC and SMC FN protein expression under atheroprone vs atheroprotective flow (n=4). (D) SMC NF- $\mathrm{kB}$-reporter activity under atheroprone vs atheroprotective flow $* P<0.05$, one-sample t-test compared to atheroprotective flow.

\subsection{Atheroprone Shear Stress Regulates SMC Inflammatory Phenotype via EC} Mechanotransduction by PECAM

Next, we tested whether the hemodynamic-induced SMC inflammatory phenotype was dependent on EC shear stress mechanotransduction through PECAM signaling. EC PECAM was knocked down with siRNA prior to re-plating onto the transwell co-culture plate (Figure 2). The ability to knock down PECAM is essential to studying its role in signaling 
inflammation to SMCs. EC PECAM was reduced to $20.9 \%$ of control after ECs were re-plated and exposed to 24 hours of flow (Figure 4A). In agreement with previous mono-culture experiments, PECAM-depleted ECs had reduced EC VCAM and FN gene expression (Figure 4B), confirming its role in the inflammatory EC phenotype in response to atheroprone shear stress.
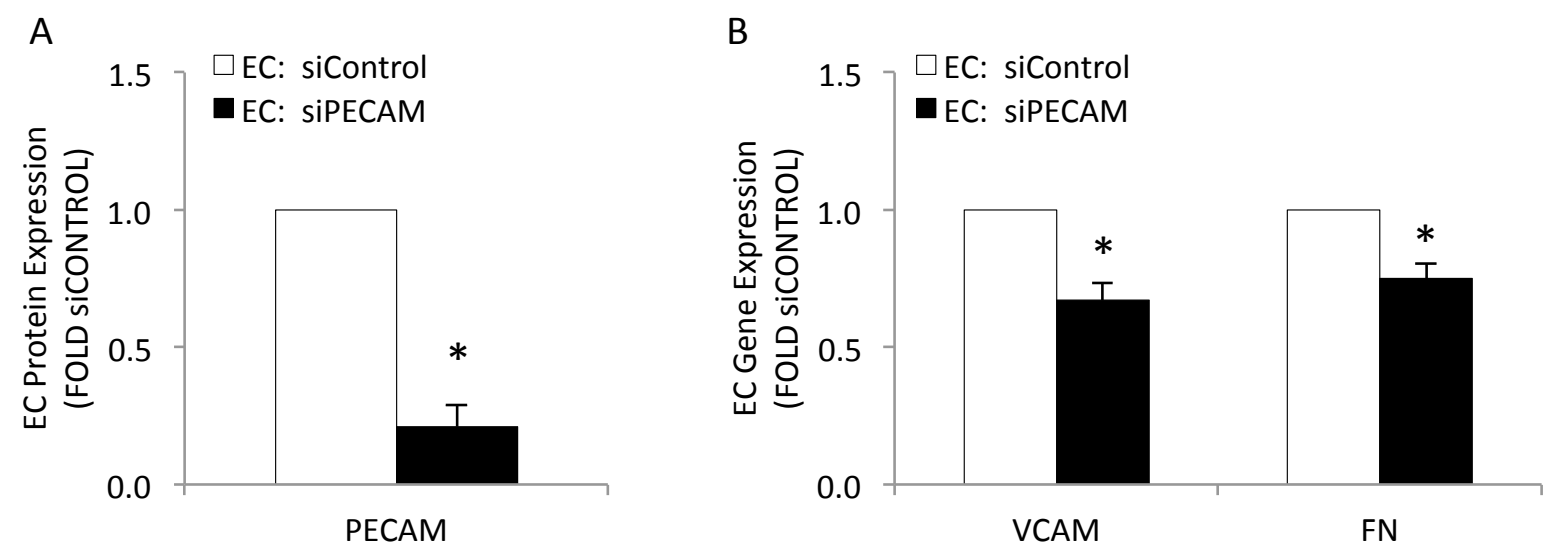

Figure 4. Mechanotransduction by EC PECAM mediates EC inflammation. (A) EC PECAM protein expression after 24 hours in the co-culture system $(n=6)$. (B) EC gene expression of VCAM and FN after EC siPECAM treatment vs siControl $(\mathrm{n}=6)$. All under atheroprone flow for 24 hours. $* P<0.05$, one-sample $\mathrm{t}$-test compared to siControl.

Endothelial PECAM was also found to be a critical regulator of the underlying SMC inflammatory phenotype in the atheroprone environment. Elevated SMC NF- $\mathrm{BB}$ activity due to atheroprone flow was greatly attenuated when co-cultured with siPECAM ECs compared to siControl (Figure 5A). Many pro-inflammatory, NF-kB-dependent genes (VCAM and Cox-2, Figure 5B) and proteins (VCAM and FN, Figure 5C) were also decreased in SMCs due to loss of PECAM. SM $\alpha$ A, a marker of SMC differentiation, increased at the protein level when EC PECAM was inhibited (Figure 5C). 
Since FN matrix is important to the signaling of an inflammatory phenotype in ECs, and exhibited flow- and PECAM-dependence in SMCs, we tested potential regulation of FN splice variants. SMCs in our system had both EDA and EDB variants present, which had been previously shown to be associated with alternative integrin binding ${ }^{107,} 108$ and macrophage infiltration ${ }^{109}$ respectively. The IIICS variant, however, was not present (data not shown). The SMC EDA FN variant was significantly increased by EC PECAM knockdown under atheroprone flow (Figure 5D, E), indicating another measure of the altered SMC phenotype in response to EC siPECAM. In ECs, only the EDA variant was expressed, and was not dependent on PECAM (data not shown). 

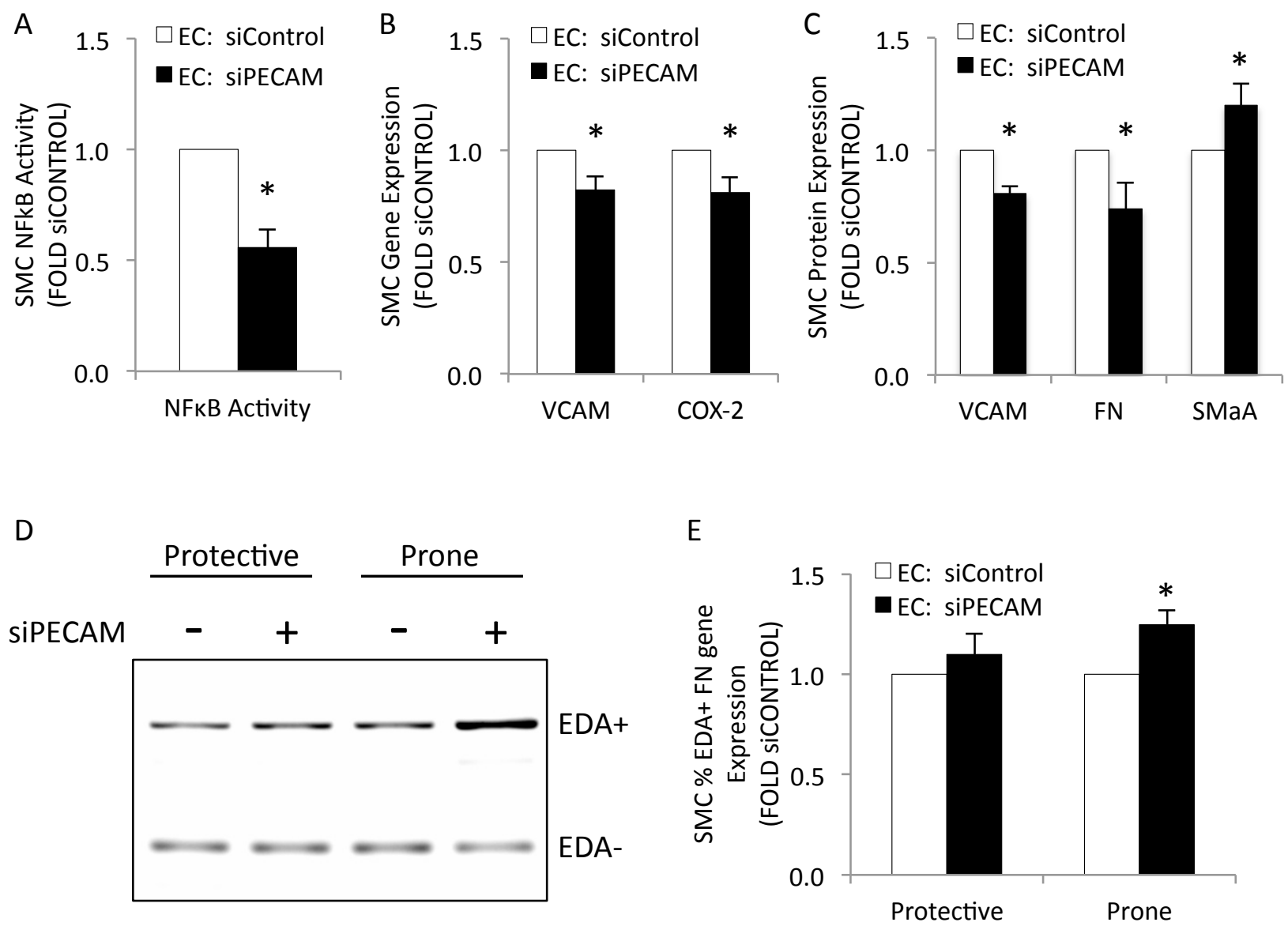

Figure 5. Atheroprone shear stress regulates SMC inflammatory phenotype via endothelial mechanotransduction by PECAM. (A) NF- $\kappa B$-reporter activity of SMCs co-cultured with EC siPECAM vs EC siControl ( $n=5)$. (B) SMC gene expression of VCAM and Cox-2 under co-cultured EC siPECAM $(n=6)$. (C) SMC protein expression of VCAM, FN, and SM $\alpha \mathrm{A}$ (n=6-12). (D) Representative DNA gel of equally loaded SMC FN PCR products resulting from primers surrounding the EDA variant site. (E) SMC FN PCR products, quantified as \%EDA+ FN gene compared to total FN (n=3). All under atheroprone flow for 24 hours unless noted Protective. $* P<0.05$, one-sample t-test compared to siControl.

\subsection{Discussion}

These data support the hypothesis that hemodynamic atheroprone and atheroprotective shear stresses indirectly regulate SMC inflammatory phenotype and that this is dependent on EC PECAM mechanotransduction. SMCs display an inflammatory cell phenotype that is marked by increased $\mathrm{NF}-\kappa \mathrm{B}$ activity in response to atheroprone flow on co-cultured ECs. This 
increase in NF- $\kappa \mathrm{B}$ activity is dependent on EC PECAM, as are flow-induced increases in VCAM and FN. The fact that SMCs are able to differentially respond to the type of shear stress applied to overlying ECs, without being in direct contact with that shear stress, indicates that ECs are actively signaling to SMCs. The current results support EC PECAM's role as a critical sensor in EC to SMC signal transduction.

An important observation in our experiments is that atheroprone flow-induced FN expression is inhibited by siPECAM in both EC and SMC. While our group previously showed that EC FN deposition is pro-inflammatory and requires PECAM, less is known about the control of FN expression by SMCs. FN is able to promote SMC NF- $\kappa \mathrm{B}$ activation in a plating concentration-dependent manner in static, IL-1 treated conditions ${ }^{81}$, and is itself dependent on $\mathrm{NF}-\kappa \mathrm{B}$ in other cell types ${ }^{64}$, creating a potential positive-feedback loop. Since we show that EC PECAM promotes both SMC $\mathrm{FN}$ and NF- $\mathrm{BB}$, this feedback loop may be sustaining inflammation in SMCs as it does in ECs. This will be investigated in Chapter 5.

The EC PECAM pathway has possible functional outcomes to the underlying SMC phenotype. SMC VCAM is PECAM-dependent, and has also been linked to activation of the transcription factor NFAT ${ }^{73}$. NFAT activity in SMCs induces proliferation and migration ${ }^{74,75}$. Proliferation and migration are associated with an inflammatory phenotype as SMC migrate into the intima and contribute to intimal thickening. SMC VCAM also promotes survival of bound leukocytes $^{110}$, and may be important to trapping leukocytes in early atherosclerotic lesions $^{111,112}$. PECAM-dependent NF- $\kappa$ B activity in SMCs also drives an inflammatory phenotype. Inhibition of NF- $\kappa \mathrm{B}$ activity in SMCs by an $\mathrm{I} \kappa \mathrm{B}$ super-repressor adenovirus results in increased SM $\alpha \mathrm{A}, \mathrm{SM} \mathrm{MHC}$, and SM22 $\alpha$ (all markers of a contractile, differentiated 
phenotype $)^{113}$. Therefore, the observed increase in NF- $\mathrm{B}$ activity decreases these differentiation markers and drives a de-differentiated phenotype in the SMCs.

SMC COX-2 is PECAM-dependent, and is found at elevated levels during inflammation. COX-2 is an enzyme responsible for prostaglandin $\mathrm{H}_{2}$ synthase, which can be further converted into prostacyclin or thromboxane. Treatment with COX-2 inhibitors show conflicting results in vascular health, suggesting that the balance between eiscosanoids downstream of COX-2 is important to vascular homeostasis ${ }^{114}$. For example, selective inhibition of COX-2 in vivo reduced intimal medial thickening and increased the contractile marker SM-MHC in $\mathrm{SMCs}^{115}$, demonstrating that COX-2 expression promotes a noncontractile and proliferative phenotype. Deletion of the thromboxane receptor delays atherogenesis in vivo by decreasing ICAM- 1 and platelet activation ${ }^{116}$, suggesting that COX-2 acting through thromboxane promotes atherosclerosis. In contrast, selective inhibition of COX2 also causes vasodilatation and SMC proliferation via prostacyclin, demonstrating that COX-2 promotes a contractile and non-proliferative phenotype ${ }^{114}$. Deletion of the prostacyclin receptor in mice is associated with inflammation and atherosclerosis ${ }^{117}$, suggesting that COX-2 acting through prostacyclin is atheroprotective. Due to these conflicting functions, further work should consider the COX-2 products prostacyclin and thromboxane individually.

An interesting finding in these experiments is the shift in the proportion of the EDA+ FN variant. Under EC siPECAM conditions SMCs display increased EDA+ FN variant, suggesting that the SMC EDA+ FN is anti-inflammatory. Inclusion of the FN type III repeat EDA (also called EIIIA, located between FN repeats $\mathrm{III}_{11}$ and $\mathrm{III}_{12}$ ) results from the inclusion of that exon during mRNA splicing ${ }^{55}$. The "classic" FN receptor, integrin $\alpha_{5} \beta_{1}$, binds at multiple sites near FN's N-terminus and at the RGD sequence in FN repeat $\mathrm{III}_{10}$. However, 
when the EDA+ variant is expressed, the EDGIHEL sequence located within it is able to bind $\alpha_{4} \beta_{1}, \alpha_{4} \beta_{7}$, and $\alpha_{9} \beta_{1}$ integrins $^{107,}{ }^{108}$ and toll-like receptor $4(\mathrm{Tlr} 4)^{118}$. In fibroblasts, EDA+ binding through $\alpha_{4} \beta_{7}$ promotes differentiation by increasing SM $\alpha$ A expression and collagen deposition $^{108}$. If SMCs also deposit more collagen when bound to EDA+ FN, this may be a mechanism for shifting from a pro-inflammatory matrix to an anti-inflammatory matrix. Indeed, EDA levels were higher in stable plaques in human patients, with stable plaques characterized with more smooth muscle cells, more collagen, and less fat ${ }^{119}$. Mouse strains devoid of EDA exon regulated splicing that are made to constitutively express EDA $^{+/+}$had decreased atherosclerotic lesion area compared to WT, however, constitutively expressing $\mathrm{EDA}^{-/-}$mice had decreased lesion area as well ${ }^{120}$. This intriguing result points to the tight control of alternative FN splicing, and further research in this area may expose a specific role for FN splicing in the context of atherosclerosis.

PECAM is well established as a mechanically-sensitive protein, responsible for regulating endothelial activation under shear stress. Here, we begin to elucidate the role of EC PECAM signaling in promoting an atheroprone phenotype in SMCs. Through PECAM, ECs are able to convey an inflammatory phenotype to SMCs in response to atheroprone shear stress, despite the fact that the shear stress is only applied to ECs. The downstream SMC phenotype has increased $\mathrm{NF}-\kappa \mathrm{B}$, which further promotes an inflammatory state over time. Further investigation into the functional consequences of this SMC phenotype may reveal therapeutic targets for preventing this inflammation from becoming chronic and atherosclerotic. 


\section{CHAPTER 5. FIBRONECTIN PROMOTES ATHEROPRONE ENDOTHELIAL AND SMOOTH MUSCLE CELL PHENOTYPES}

\subsection{Introduction}

FN has been implicated in the development of atherosclerosis in vivo, with increased deposition in atheroprone segments of the abdominal aorta $^{64}$. FN is visible both at the endothelium and the intimal extracellular matrix, which contains SMCs. Furthermore, this deposition correlates with focal increases in inflammatory VCAM staining. Focal FN deposition has been confirmed in the atheroprone lesser curvature region of the aorta, where it was seen beneath the endothelial layer ${ }^{64}$ and is dependent on PECAM $^{48}$.

Previous data has shown that ECs subjected to shear stress in vitro display greater FN expression under atheroprone flow than those under atheroprotective flow ${ }^{48}$. ECs under atheroprone flow also have increased inflammatory NF- $\mathrm{BB}$ activity when FN is present. Regulation of FN in the SMC layer, however, is not well understood. In Chapter 4, we learned that EC and SMC FN are dependent on atheroprone shear stress and EC PECAM. Since PECAM was a significant regulator of SMC phenotype, we hypothesized that EC FN may also regulate SMC phenotype. In this chapter, we sought to determine the regulatory role of FN in promoting endothelial and smooth muscle cell atheroprone phenotypes. We hypothesized that expression in either cell types acts as a positive regulator of inflammation.

To investigate the role of EC FN, we used siRNA to knock down FN in ECs prior to replating into the co-culture system. After ECs were exposed to 24 hours of atheroprone shear stress, the SMC inflammation was measured. Knockdown of FN in mono-cultured ECs attenuates inflammation in response to atheroprone flow, as indicated by EC NF- $\mathrm{BB}$ activity. NF- $\mathrm{kB}$ is a key transcriptional factor implicated in inflammation, and contributes to SMCs' 
ability to proliferate ${ }^{121}$. Therefore, NF- $\mathrm{KB}$ activity was an important measure of inflammation in SMCs. Another functional measure of inflammation in SMCs was monocyte adhesion. Our work in Chapter 4 showed that atheroprone flow induced expression of VCAM in SMCs relative to atheroprotective flow. VCAM is known to play a role in monocyte adhesion ${ }^{122}$, so monocyte adhesion assays were used to assess the functional consequences of VCAM expression.

To investigate the role of SMC FN, we performed additional experiments using siRNA to knock down FN in SMCs prior to re-plating into the co-culture system. Re-plating after transfection was important so that the SMCs could secrete a new matrix with knocked-down FN, rather than remaining on the matrix they deposited before the siRNA transfection. After SMCs were re-plated, ECs were plated in co-culture as described in Chapter 3.1, and atheroprone shear stress was applied to the ECs for 24 hours. Inflammation was then measured

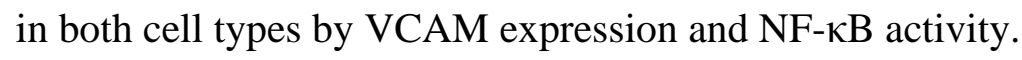

\subsection{Endothelial FN Promotes SMC Inflammatory Phenotype}

We first studied the role of EC FN in promoting an inflammatory SMC phenotype. Loss of EC FN (17.7\% $\pm 1.8 \%$ of control, $\mathrm{n}=5)$ caused a significant decrease in SMC NF- $\kappa \mathrm{B}$ activity (Figure 6A). Interestingly, when exogenous FN was added to the media of siFN ECs for the final 8 hours of the experiment, the SMC NF- $\kappa B$ response was completely rescued (Figure 6A). Similar to the regulation by EC PECAM, loss of EC FN also significantly reduced the expression of the NF- $\kappa$ B-dependent gene COX-2 and protein VCAM (Figure 6B, C). KLF4 gene expression had a slight but significant increase with EC FN knockdown (Figure 6B). FN is a secreted protein that may pass through the permeable polycarbonate membrane $(0.4 \mu \mathrm{m}$ 
pore size) separating the cell types, but permeation does not appear to occur since knocking down FN in ECs had no effect on either SMC FN gene or protein (Figure 6B, C). This indicates that the regulation of SMC phenotype by EC FN is specific only to the EC FN and that, in contrast to EC PECAM regulation of SMC NF- $\mathrm{BB}$, phenotypic changes occur through signaling independent of SMC FN.

Previous work from our lab has shown that SMCs exhibit a 2-fold greater monocyte adhesion when co-cultured ECs were under atheroprone shear stress compared to atheroprotective $^{72}$. To determine the ability of EC FN to modulate the functional adhesion of monocytes to the SMCs, adhesion assays were performed following exposure to atheroprone hemodynamics (Figure 6D-F). The average number of arrested monocytes per field of view decreased by $30.7 \%$ with EC siFN treatment (Figure 6F). These results demonstrate that atheroprone-mediated monocyte adherence to SMCs is dependent on EC FN. 

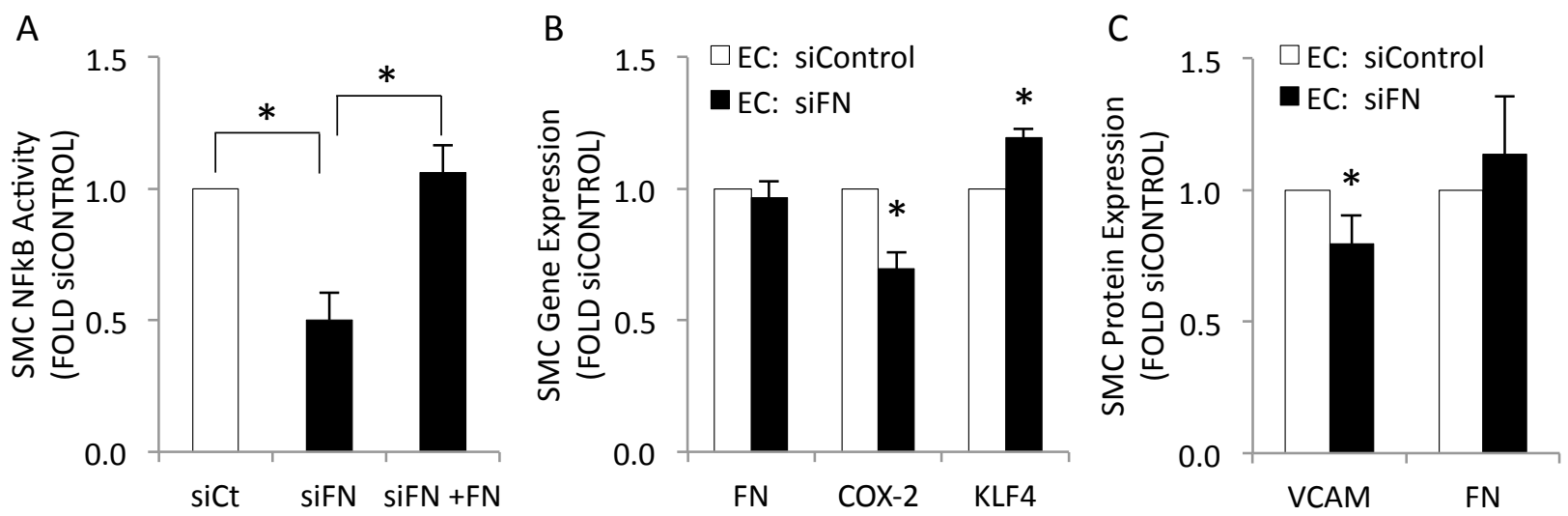

D

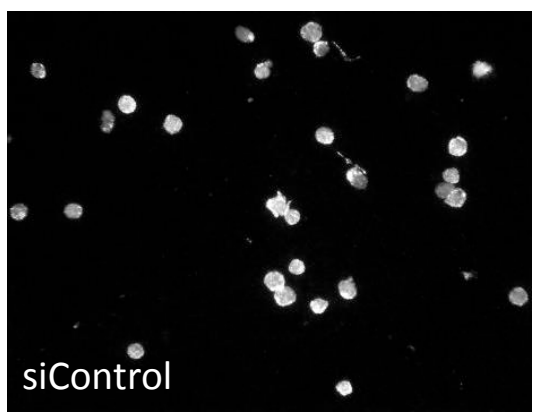

$\mathrm{E}$

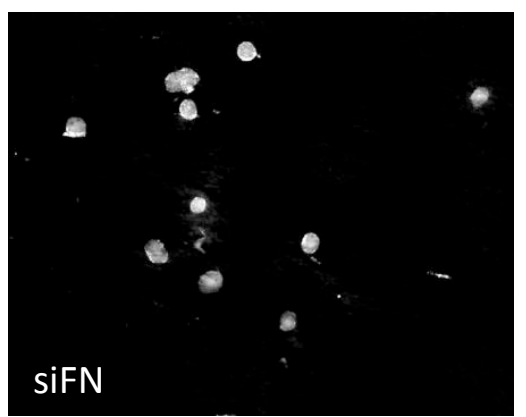

$\mathrm{F}$

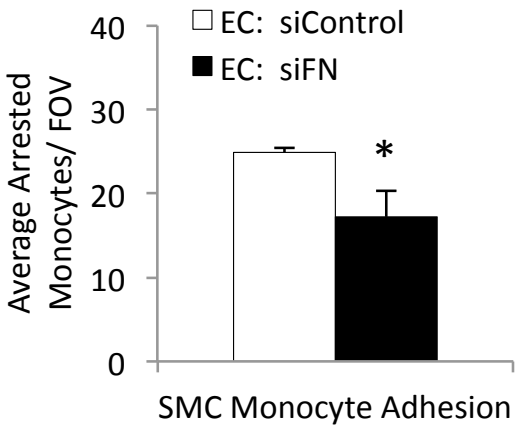

Figure 6. Endothelial FN deposition promotes an inflammatory phenotype in SMCs. (A) NF-кBreporter activity of SMCs co-cultured with EC siFN or siFN + exogenous FN media vs EC siControl ( $\mathrm{n}=4-5)$. (B) SMC gene expression of FN, COX-2, and KLF4 under co-cultured EC siFN (n=3). (C) SMC protein expression of VCAM and FN ( $\mathrm{n}=5$ ). (D and E) Representative images of SMC monocyte adhesion assay under EC siControl or siFN conditions. (F) Arrested monocytes averaged across 10 images $(n=3)$. All under atheroprone flow for 24 hours. ${ }^{*} P<0.05$, one-sample t-test compared to siControl, student's t-test for siFN compared to siFN+FN, and paired t-test for monocyte adhesion assay.

\subsection{SMC FN Promotes Positive Feedback of EC Inflammatory Phenotype}

Since we previously showed that SMC FN is regulated by atheroprone flow (Figure 3B), we next performed experiments to determine the role of SMC FN in promoting inflammatory phenotypes. A 49\% FN knockdown in SMC corresponded to a decrease in SMC VCAM, indicating the ability for FN to promote inflammation in SMCs (Figure 7A). This SMC FN knockdown caused a subsequent decrease in EC VCAM, suggesting that the 
inflammatory matrix environment of SMCs provides positive feedback to further inflame the co-cultured ECs (Figure 7B). Similar to EC siFN experiments, SMC siFN experiments did not change EC FN expression (Figure 7B), so the change in EC inflammation is signaled through an EC FN-independent method. Finally, NF- $\kappa \mathrm{B}$ activity in both SMCs and ECs was significantly decreased under SMC siFN, pointing to the importance of SMC FN in promoting both SMC and EC inflammatory phenotypes (Figure 7C).

A

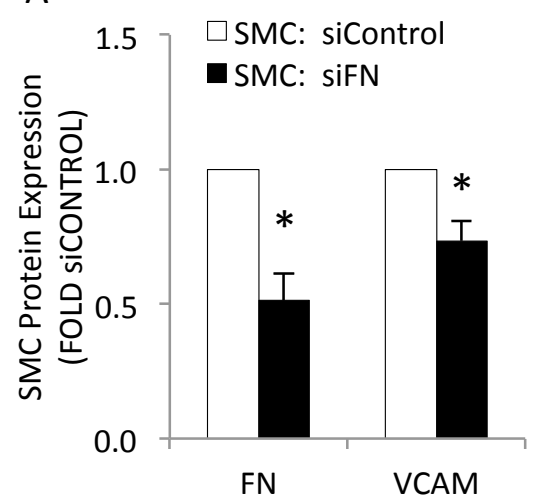

B

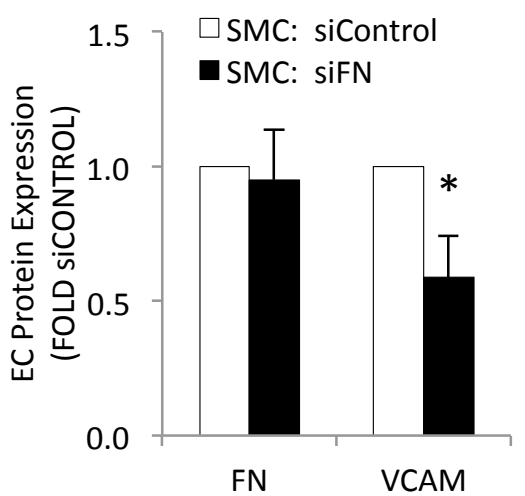

C

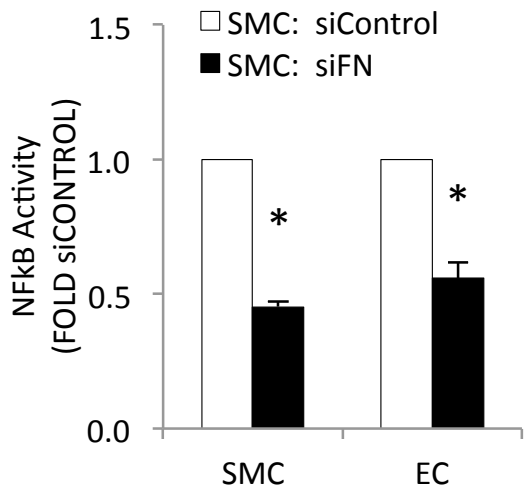

Figure 7. Shear stress-induced SMC FN promotes inflammation in SMCs and ECs. (A) SMC protein expression of FN and VCAM after SMC siFN or siControl treatment (n=7-9). (B) EC protein expression of FN and VCAM after SMC siFN or siControl treatment $(n=7-9)$. (C) NF- $\mathrm{kB}$-reporter activity of SMCs and ECs co-cultured with SMC siFN or siControl $(n=3)$. All under atheroprone flow for 24 hours. $P<0.05$, onesample t-test compared to siControl.

\subsection{Discussion}

FN deposition by ECs and SMCs is dependent on atheroprone shear stress. These data support the hypothesis that FN expression by both cell types acts as a positive regulator of inflammation. EC FN promotes SMC VCAM expression and monocyte adhesion, as well as COX-2 gene expression. EC FN also drives SMC inflammatory NF- $\kappa \mathrm{B}$ activity, whether from the ECs themselves or from addition of exogenous FN to the EC media. This inflammatory SMC phenotype occurs without changes to the SMC FN matrix. Similarly, SMC FN promotes 
SMC and EC VCAM expression and NF- $\mathrm{BB}$ activity. The resulting inflammatory EC phenotype occurs without changes to the EC FN matrix. These data suggest that FN is not deposited as a homogenous FN matrix that is shared between the cell types. Rather, the inflammatory phenotype of either cell can be influenced without its FN matrix changing, suggesting that the inflammatory signal is conveyed through another method.

Recovery of SMC NF- $\mathrm{kB}$ activity with the addition of exogenous FN to EC media was especially exciting. This restoration of inflammation in ECs, and subsequent signaling to SMCs, alludes to ECs' ability to incorporate the exogenous FN into their matrix. Shear stress is known to trigger conversion of integrins to their high affinity state prior to binding $\mathrm{ECM}^{123}$. Soluble FN selectively binds and interacts with integrin $\alpha_{5} \beta_{1}$ but not other RGD-depedent integrins ${ }^{124} \cdot \alpha_{5} \beta_{1}$ is expressed on the apical surface of $\operatorname{HUVEC}^{125}$, and therefore able to bind exogenous FN. Once bound, it can induce integrin clustering into focal adhesions to promote fibrillar matrix formation or undergo integrin-mediate degradation by endocytosis with caveolin- $1^{126}$. When integrins are bound to ECM under shear stress, they mediate cytoskeletal alignment and NF- $\mathrm{KB}$ activity through the GTPase Rac1 ${ }^{127}$. In this experiment, FN remained bound to integrins long enough to sufficiently recover the inflammatory EC signaling output, leading to SMC NF-kB activity.

Another revelation gleaned from the experiments with exogenous FN in EC media is the lack of significance of splice variants in EC to SMC signaling. Exogenous FN added to EC siFN experiments completely recovered downstream SMC NF- $\mathrm{kB}$ activity, indicating that the EC inflammatory pathways were restored by exogenous FN. Exogenous FN was originally purified from human plasma. Plasma FN is a more soluble form of FN than cellular FN, and lacks any of the splice variants potentially observed in cellular $\mathrm{FN}^{55}$. So while SMC FN 
variants were a marker of its phenotypic modulation (Chapter 4), FN splice variants do not have a role in atheroprone EC-SMC inflammatory signaling.

This work is the first, to our knowledge, to study the role of FN in co-culture. Another co-culture model has shown that ECs exposed to either TNF $\alpha$ or laminar shear stress have lower inflammatory marker expression when co-cultured with SMCs compared to monoculture $^{87}$. However, under atheroprone flow conditions SMCs provide inflammatory feedback to ECs, and that FN. Knockdown of SMC FN-mediated feedback had the expected result of decreasing SMC inflammation. SMC plating on FN matrix is well established to promote a synthetic phenotype with higher proliferation and reduced contractile markers ${ }^{128-130}$. It was not known, however, that the FN matrix regulates the SMC inflammatory signaling output. We show that SMC-FN interactions promote inflammation in ECs despite an unchanged EC matrix. This creates a positive-feedback loop in which shear stress-induced EC FN deposition promotes SMC inflammation, and SMC FN promotes EC inflammation in return (Figure 8). An EC-SMC feedback loop could produce sustained inflammation, leading to the chronic conditions associated with atherosclerosis. 


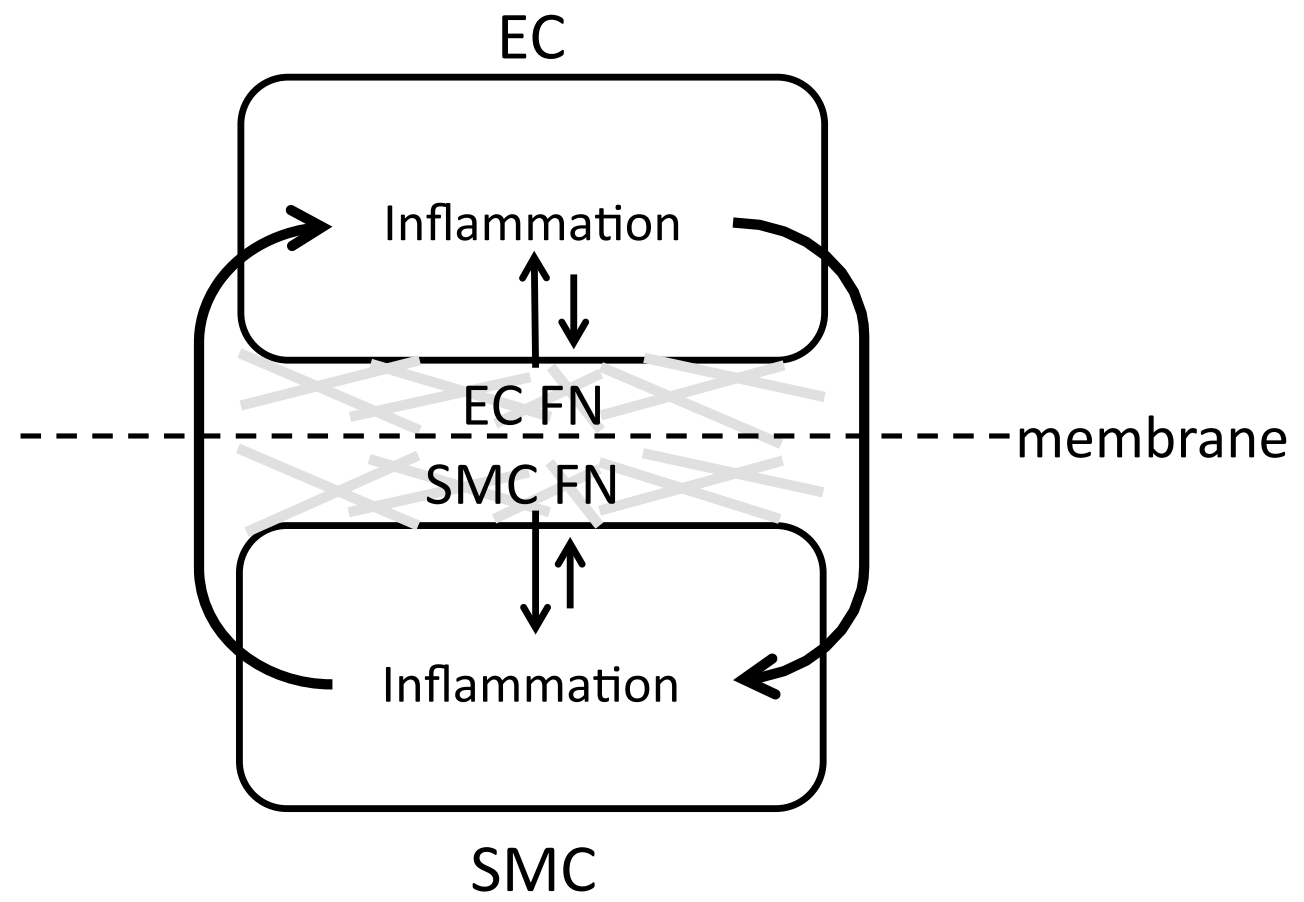

Figure 8. Proposed EC-SMC FN inflammation feedback loop.

Monocyte adhesion to SMCs is a relevant functional measure of EC FN-dependent inflammation. Monocyte adhesion to the endothelium and subsequent transmigration across the endothelium into the intima is a key early step in the development of atherosclerosis. Once recruited, monocytes in the vessel wall produces cytokines such as TNF $\alpha$ to further propagate the disease ${ }^{9}$. Due to VCAM's known role in monocyte adhesion ${ }^{122}$, and its regulation by EC and SMC FN, monocyte adhesion was used to assess the functional consequences of VCAM expression. ECs are the traditional target for monocyte adhesion, because they are the first line of defense at the vessel wall. SMCs, however, may aid in the accumulation of transmigrated monocytes within the vasculature. SMC VCAM expression is detected in an atherosclerotic rabbit model several weeks after detection in the endothelium ${ }^{131}$. This fits with a model of early 
monocyte adhesion by ECs, followed by later retention via SMCs. Our results confirm that monocyte adhesion to SMCs is dependent on the inflammatory cell phenotype, downstream of EC FN signaling.

A FN-rich matrix is ideal for tissue formation and vascular remodeling, but a lasting presence of this matrix leads to inflammation and disease. In this chapter, we show that FN promotes inflammation in the ECs and SMCs it supports. Not only does FN contribute to the atheroprone phenotype of the cells expressing it, but it also promotes paracrine inflammatory signaling mechanisms. FN deposition by ECs is PECAM dependent, and the SMC phenotype derived from EC siFN matches that of EC siPECAM, emphasizing that the pathway of EC PECAM signaling occurs through FN. Through this inflammatory pathway, atheroprone shear stress applied to ECs drives a positive feedback loop of FN deposition between ECs and SMCs, leading them to chronically inflamed states. 


\section{CHAPTER 6. SECRETED PROTEINS PROMOTE SMOOTH MUSCLE CELL INFLAMMATION}

\subsection{Introduction}

ECs and SMCs are able to produce and respond to secreted proteins (e.g., cytokines, growth factors), making these short-range mediators a potential signaling mechanism between cell types downstream of the PECAM/FN pathway. A variety of pro-inflammatory cytokines have been found in atherosclerotic plaques $^{121}$. Pro-inflammatory cytokine expression is regulated by $\mathrm{NF}-\kappa \mathrm{B}$ activity, which is highly regulated by atheroprone flow, PECAM, and $\mathrm{FN}^{48}$. In particular, shear stress is known to differentially regulate EC secretion of IL- $1^{101}$, IL$6^{95}$, and IL-8 ${ }^{96,97}$. In vivo models also show regulation of MCP-1, GRO- $\alpha$, and fractalkine by shear stress ${ }^{98}$. Since the impact of EC FN signaling found in Chapter 5 was likely not direct, we hypothesized that FN-dependent secreted factors act as the signaling mechanism promoting EC-SMC inflammation. In this aim, we collected cell media and screened for secreted proteins that regulate the atheroprone SMC phenotype. We hypothesized that the atheroprone phenotype is modulated by secreted proteins downstream of the PECAM/FN pathway.

First, we sought to determine whether there are differences in the media of ECs from siControl versus siPECAM or siFN conditions that would promote a pro-inflammatory response in SMCs. Static SMCs were treated with control or flow-conditioned media to study the effects of factors secreted into the media without any physical interaction with ECs. Next, to study the role of conditioned media in the co-culture system, we performed a recovery experiment. The out-flow media from an atheroprone EC siControl experiment was pumped 
into an atheroprone EC siFN experiment. Thus, the ECs in co-culture had FN knocked down, but were supplemented with media with control ECs.

Finally, we used a multiplexing approach to measure secreted factors from the cell culture media to identify possible cytokines that were differentially regulated. Secreted concentrations of 19 cytokines were measured, and of those, EC FN knockdown regulated 12 cytokines. GRO- $\alpha$ specifically had the ability to induce NF- $\kappa \mathrm{B}$ activity in SMCs, and may be an important mediator of the SMC inflammatory response under shear stress.

\subsection{Endothelial Secreted Factors Regulate SMC Inflammatory Phenotype}

To determine whether secreted factors in the cell media were able to influence SMC inflammation without the physical presence of ECs, we first performed experiments with static SMCs. The static SMCs were plated in serum-starvation to reach quiescence, then cultured for 24 hours with control media or conditioned media collected during the last 8 hours of atheroprone flow experiments. Treating with siControl media increased SMC NF- $\kappa$ B activity, indicating that this media was able to signal the atheroprone condition to the static SMCs

(Figure 9A). In contrast, the atheroprone phenotype in static SMCs was inhibited by culture with siPECAM of siFN conditioned media (Figure 9A, B). This is evidence that atheroprone shear stress induces differences in the composition of secreted proteins that regulate the inflammatory SMC phenotype. Further, these secreted proteins are dependent on signaling through EC PECAM and FN.

To test the influence of secreted factors during a real-time shear stress experiment, we created a new media pumping method. Rather than pumping fresh flow media into the EC siControl or siFN co-culture dishes, conditioned media from a parallel control atheroprone 
experiment was pumped directly from the control dish into the experimental dish. This allowed us to re-create the inflammatory secreted milieu on top of FN knock-down ECs. In the EC siFN and $\mathrm{EC}$ siFN+ siControl media experiments, FN gene expression was knocked-down to $17.7 \%$ $\pm 1.4 \%$ and $20.9 \% \pm 4.0 \%$ of control, respectively. Despite the continued FN knock down, experiments with siFN+ siControl media completely rescued underlying SMC inflammatory NF- $\kappa \mathrm{B}$ activity (Figure 9C). Thus, secreted factors in the media are dependent on EC PECAM and FN, and are capable of conferring SMC inflammation regardless of the whether EC FN is present.
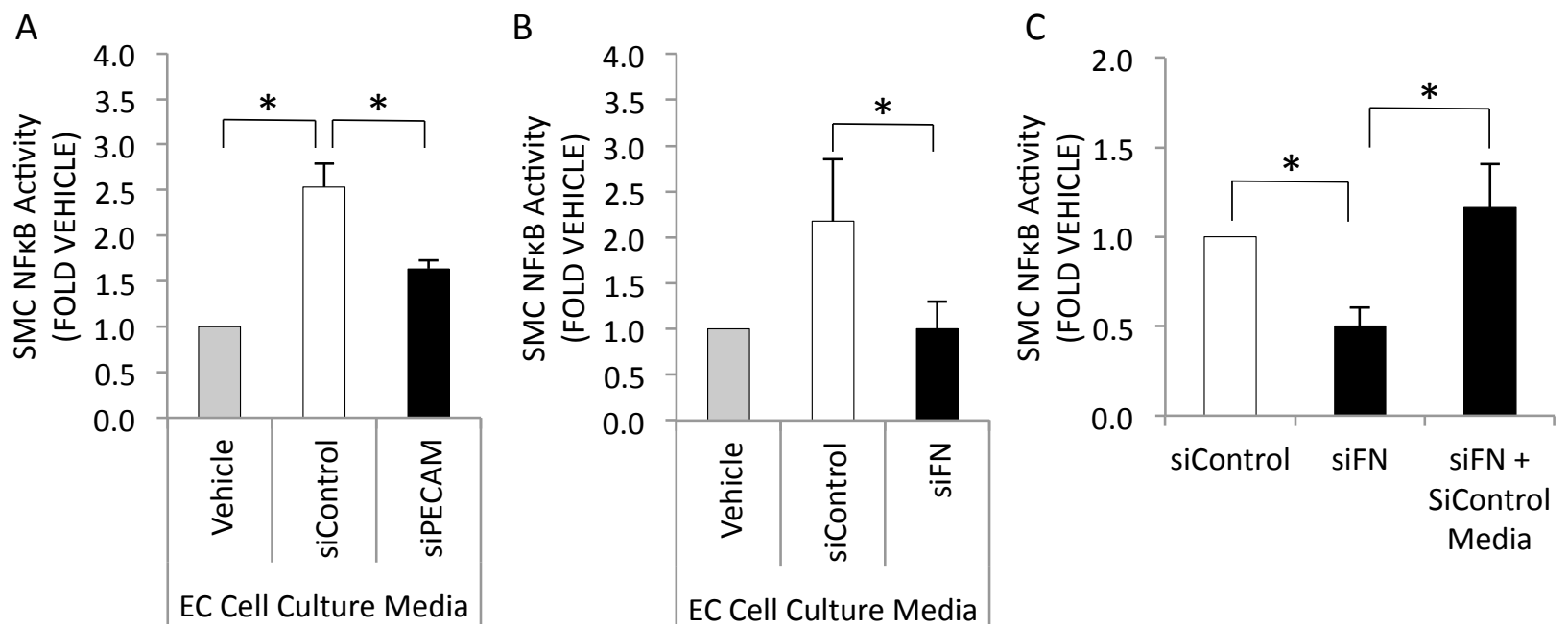

Figure 9. Endothelial secreted factors regulate SMC phenotype. (A) NF-kB-reporter activity of static SMCs treated with conditioned media for 24 hours. EC conditioned was media taken from last 8 hours of EC siControl or siPECAM atheroprone flow experiments $(n=3)$. (B) NF- $\kappa B$-reporter activity of static SMCs treated with conditioned media for 24 hours. EC conditioned was media taken from last 8 hours of EC siControl or siFN atheroprone flow experiments $(n=4)$. (C) NF- $\kappa B$-reporter activity of SMCs under atheroprone flow, co-cultured with EC siFN vs EC siControl, and with continuous addition of media pumped directly from a parallel control experiment $(\mathrm{n}=3-5)$. $* P<0.05$, one-sample t-test compared to siControl or vehicle, student's t-test compared between media conditions. 


\subsection{Endothelial Secreted Proteins are Modulated by FN}

Our static SMC experiments confirmed that an EC secreted factor (or factors) regulates the SMC phenotype downstream of EC FN. To determine what this factor (or factors) may be, we used a multiplex screening approach to measure secreted factors. The Luminex MAGPIX system with a Milliplex cytokine assay kit allowed for the measurement of up to 41 analytes per sample in a single plate well. We chose to measure 19 analytes that are expressed by ECs. Of the 19 cytokines analyzed, we measured detectable levels of 13. Average secretion concentrations from siControl experiments are summarized in Table 2.

\begin{tabular}{|c|c|}
\hline Cytokine & $\begin{array}{c}\text { Secretion } \\
\text { (Average pg/mL } \pm \text { SE) }\end{array}$ \\
\hline$\overline{\mathrm{MCP}-1}$ & $4033.8 \pm 719$ \\
\hline IL-8 & $401.3 \pm 112$ \\
\hline $\operatorname{GRO} \alpha$ & $230.5 \pm 54.1$ \\
\hline IL-6 & $46.77 \pm 6.38$ \\
\hline PDGF AA & $43.72 \pm 2.84$ \\
\hline FRACTALKINE & $19.19 \pm 1.75$ \\
\hline VEGF & $15.38 \pm 1.34$ \\
\hline SCD40L & $5.55 \pm 0.41$ \\
\hline $\mathrm{MDC}$ & $4.47 \pm 0.35$ \\
\hline G-CSF & $4.04 \pm 0.47$ \\
\hline GM-CSF & $1.32 \pm 0.22$ \\
\hline IFN $\gamma$ & $0.97 \pm 0.05$ \\
\hline MIP1 $\beta$ & $0.70 \pm 0.11$ \\
\hline EGF & ND \\
\hline IL-7 & ND \\
\hline I-12P40 & ND \\
\hline$\underline{\mathrm{L}-13}$ & $\mathrm{ND}$ \\
\hline MIPl $\alpha$ & $\mathrm{ND}$ \\
\hline RANTES & ND \\
\hline
\end{tabular}

Table 2. Measured cytokine secretion from siControl atheroprone flow experiments, measured by Luminex MAGPIX (n=10-12). ND: not detected. 
We discovered that 12 of the 13 cytokines measured under atheroprone flow were differentially regulated by EC siFN (Figure 10A). This overwhelming response showed that it was not a single cytokine responsible for signaling EC-SMC inflammation. Rather, EC FN is able to influence the secretion of many cytokines, leading to a complex pro-inflammatory milieu.

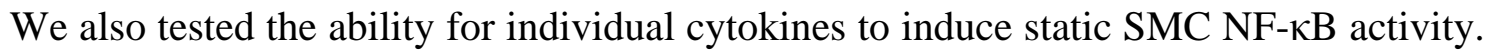
MCP-1, GRO- $\alpha$, and PDGF-AA are secreted at high concentrations (Table 2), and have wellrecognized receptors on SMCs. Of these three, only GRO- $\alpha$ was found to increase SMC NF- $\mathrm{BB}$ activity with a dose-dependent response (Figure 10B). The upregulation of NF- $\mathrm{B}$ activity under GRO- $\alpha$ treatment requires plating on a pro-inflammatory $\mathrm{FN}$ matrix, with plating on gelatin failing to induce a response (Figure 10B). Therefore, we hypothesize that EC GRO- $\alpha$ is an important mediator of the atheroprone SMC phenotype, but is not alone sufficient. When pre-conditioned media from siControl atheroprone experiments was applied to static SMCs on gelatin, the cytokine mixture (which includes GRO- $\alpha$ ) induces NF- $\kappa$ B activity (Figure 10C). Therefore, one of the other secreted factors in pre-conditioned media is able to "prime" the SMCs for a response, as the plating on FN did. Finally, inhibiting the GRO- $\alpha$ receptor CXCR2 in SMCs inhibits the increase in $\mathrm{NF}-\kappa \mathrm{B}$ activity under pre-conditioned media (Figure 10C). This decrease in $\mathrm{NF}-\kappa \mathrm{B}$ activity with only GRO- $\alpha$ receptor inhibition gives evidence for the importance of GRO- $\alpha$ signaling within the inflammatory cytokine environment. 

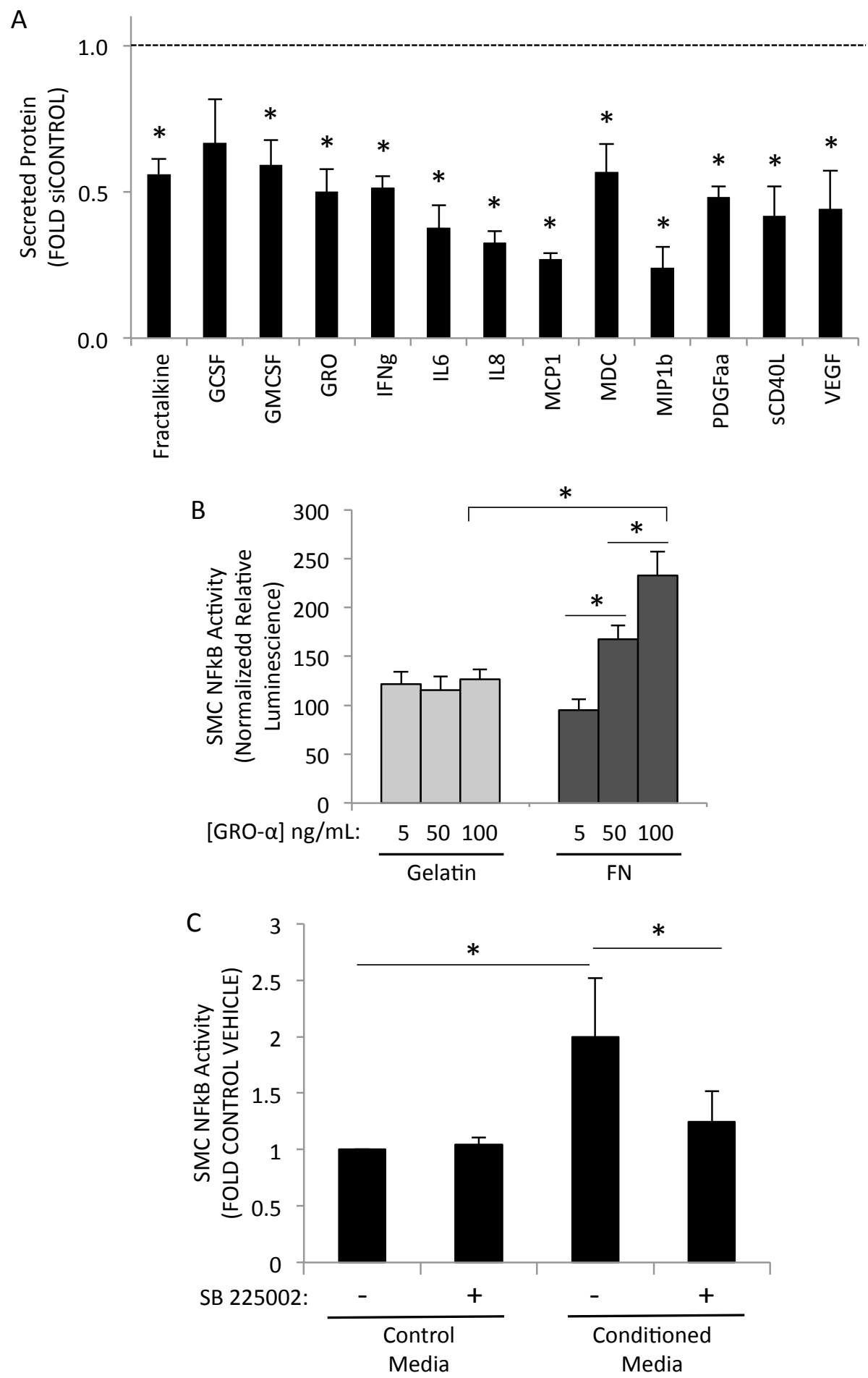

Figure 10. FN-dependent endothelial secreted proteins regulate SMC phenotype. (A) Protein secreted into media during EC siFN vs siControl atheroprone flow experiments, measured by Luminex MAGPIX $(\mathrm{n}=4)$. (B) NF-кB-reporter activity of static SMCs plated on gelatin or FN. SMCs were treated with human GRO- $\alpha$ cytokine 4 hours before experiment take-down $(n=4)$. (C) NF- $\kappa B$-reporter activity of static SMCs plated on gelatin, pretreated with CXCR2 inhibitor SB 225002. EC conditioned was media taken from last 8 hours of EC siControl atheroprone flow experiments and applied to static SMCs for 24 hours $(\mathrm{n}=5) * P<0.05$, one-sample t-test compared to siControl, student's t-test compared between cytokine treatment conditions. 


\subsection{Discussion}

Our data provides evidence that the atheroprone phenotype is modulated by secreted proteins downstream of the EC PECAM/FN pathway. Factors secreted into the media during atheroprone flow experiments are sufficient to upregulate SMC NF- $\kappa$ B activity without the physical presence of ECs. Furthermore, these secreted factors are dependent on EC PECAM and FN. During co-culture EC siFN flow experiments, the FN-dependent secreted factors could be rescued using a parallel co-culture experiment to restore SMC NF- $\mathrm{B}$ activity. When we screened secreted media, we found that $\mathrm{FN}$ regulates secretion of an entire cytokine milieu. Of these cytokines, GRO- $\alpha$ specifically could induce inflammation of SMCs plated on FN. CXCR2, the GRO- $\alpha$ receptor, is expressed throughout the SMC-containing intima and media in vivo ${ }^{72}$ making it physiological relevant signaling mechanism.

The ability for GRO- $\alpha$ to modulate the SMC phenotype was particularly interesting. GRO- $\alpha$ has no known affect on SMCs. However, it is expressed in atherosclerotic lesions, and recruits monocytes ${ }^{132,}$ 133. In a vascular injury model, inhibiting GRO- $\alpha$ with a blocking antibody had the affect of impairing endothelial recovery, but SMCs were unaffected ${ }^{134}$. GRO$\alpha$ does have the ability to induce chemotaxis and proliferation in ECs in vitro ${ }^{135}$, so a similar mechanism may allow it to induce proliferation in SMCs.

GRO- $\alpha$ was unable to induce SMC NF- $\mathrm{B}$ activity on gelatin. However, conditioned media from flow experiments was able to induce NF- $\kappa$ B activity. This suggests that at least one of two possible components are missing from the single GRO- $\alpha$ treatment to induce inflammation. First, additional cytokines may be required to induce FN expression before GRO- $\alpha$ can have an effect. This would explain why SMCs plated on FN had increased NF- $\kappa$ B 
activity with GRO- $\alpha$ treatment alone. The requirement for additional cytokines is also plausible, because many other cytokines were both secreted and differentially regulated. Second, the SMCs may require their own inflammatory "priming" before being induced by GRO- $\alpha$. In this case, FN was able to prime the SMCs. While gases such as NO are secreted by ECs and regulate SMC phenotype ${ }^{91}$, NO is vasodilatory. NO also has a half-life of about 7 minutes when hemoglobin is not present ${ }^{136}$, so it and other gases are not likely to remain in the media for the time required to aliquot it or through the freeze-thaw process. Another possible source of SMC "priming" could be the release of miRNAs from ECs. MiR-221 and miR-222 are able to increase SMC growth and proliferation in vitro ${ }^{137}$, and are highly expressed in $\mathrm{ECs}^{138}$. While shear stress-induced EC miR-143/145 promotes SMC differentiation ${ }^{92}$, there may be undiscovered shear stress-induced miRNAs that promote inflammatory "priming" of SMCs.

Previous work from our lab involving the cytokine IL-8 also stresses the importance of an entire array of secreted proteins in promoting SMC inflammation. In the co-culture system under atheroprone flow, IL-8 had increased secretion ${ }^{72}$. However, this IL-8 had the effect of increasing SM $\alpha \mathrm{A}$ and decreasing VCAM, which is opposite of the result we achieve with the entire mix of cytokines. Signaling from other cytokines may out-weigh that of the protective effects of IL-8. Or, because IL-8 and GRO- $\alpha$ may both bind the CXCR2 receptor, competition by GRO- $\alpha$ may inhibit the IL-8 binding kinetics.

EC FN-dependent cytokines provide the missing link between EC and SMC atheroprone phenotypes. A summary of the proposed signaling pathway is shown in Figure 11. We have demonstrated that EC PECAM senses disturbed shear stress and upregulates FN deposition and VCAM expression. This inflammatory EC phenotype with increased FN was 
previously shown to be regulated by $\mathrm{EC} N \mathrm{NF}-\mathrm{B}$ in mono-culture ${ }^{48}$. EC VCAM expression allows monocyte adhesion, and cytokines secreted in our system have roles in firm adhesion (MCP-1 and IL-8) ${ }^{139}$, extravasation (MCP-1 and IL-8) ${ }^{140}$, and differentiation into macrophages $(\mathrm{GM}-\mathrm{CSF})^{141}$. Inflammation in ECs also drives SMC inflammation, and we now propose that this occurs through secreted factors such as cytokines (Figure 11-1). Cytokines secreted by activated ECs are regulated by FN, and are able to induce an SMC phenotype with increased $\mathrm{NF}-\kappa \mathrm{B}$ activation despite the lack of physical EC presence (Figure 11-2). The SMC inflammatory phenotype also expresses VCAM and FN, which could be driven through NF- $\mathrm{KB}$ activation or other transcription factors. SMCs convey an inflammatory signal back to ECs in a FN-dependent fashion (Figure 11-3). SMC FN regulates both SMC and EC NF- $\kappa$ B activity.

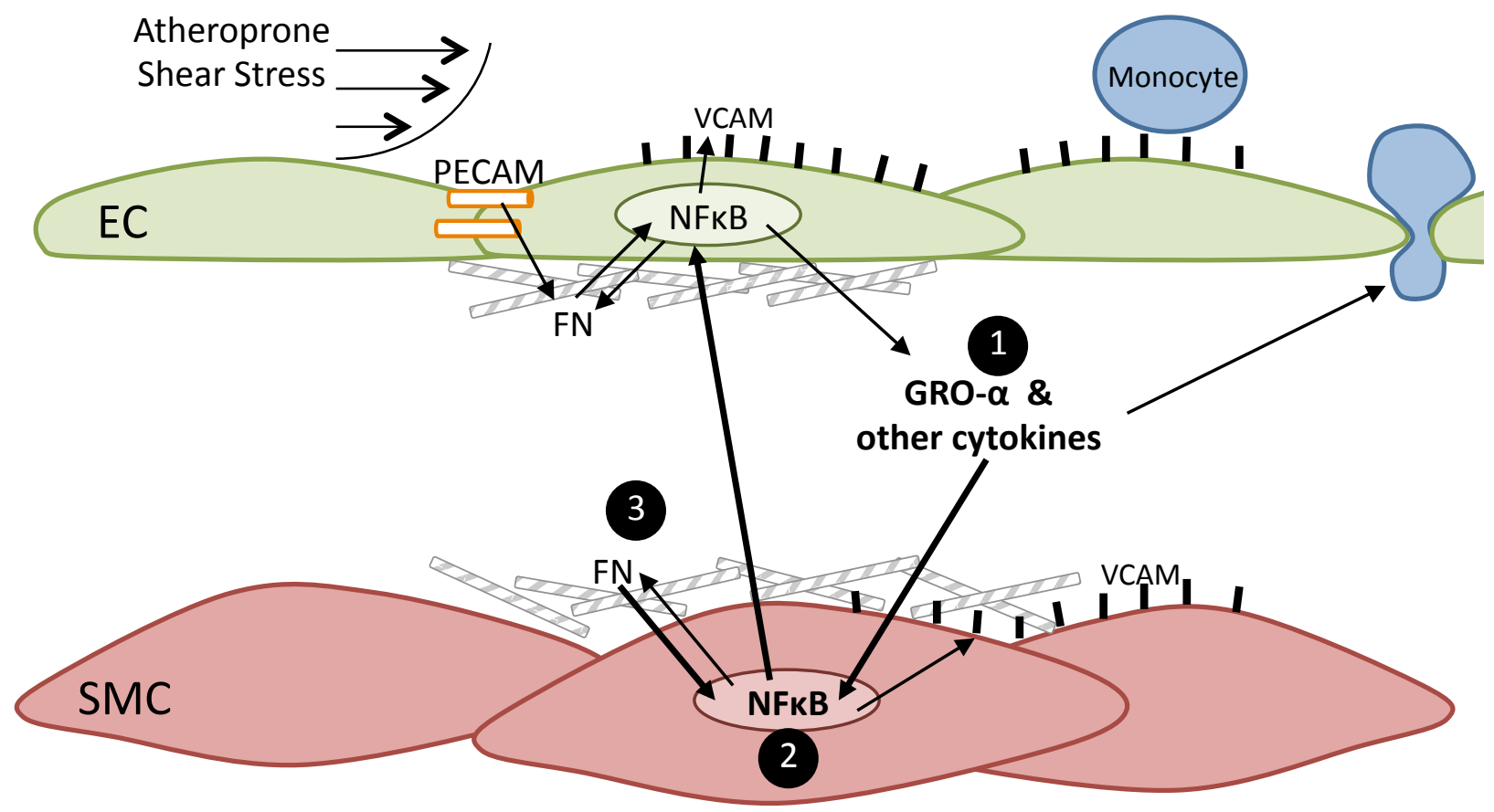

Figure 11. Proposed signaling pathway. Atheroprone shear stress on ECs is sensed by PECAM to increase FN, inflammatory NF- $\kappa B$ activity, and VCAM expression. (1) EC-secreted GRO- $\alpha$ and other cytokines are differentially regulated by FN, potentially by the FN-dependent activity of EC NF- $\kappa$ B. Cytokines increase SMC NF- $\kappa B$ activity and aid in the adhesion and extravasation of monocytes. (2) Increased SMC NF- $\kappa B$ activity promotes inflammation, through increased expression of FN and VCAM. (3) SMC FN further promotes NF- $\mathrm{\kappa B}$ activity in SMCs, and this inflammatory phenotype is signaled to ECs through regulation of $\mathrm{NF}-\kappa \mathrm{B}$. 


\section{CHAPTER 7. KRUPPEL-LIKE FACTOR 4 ESTABLISHES AN}

\section{ATHEROPROTECTIVE ENDOTHELIAL PHENOTYPE}

\subsection{Introduction}

Given that endothelial activation can result in vascular disease, identifying molecular pathways that mediate this response is of great therapeutic interest. Dr. Mukesh Jain's lab at Case Western Reserve University has previously suggested the atheroprotective role of endothelial KLF4 using steady shear stress and cytokine stimulation ${ }^{103}$. KLF4 is expressed in human and mouse ECs in vitro and in vivo, and overexpression of KLF4 induces antiinflammatory and anti-thrombotic factors such as eNOS and thrombomodulin (TM). This antiinflammatory phenotype has the functional ability to inhibit $\mathrm{TNF} \alpha$-induced monocyte adhesion. Knockdown of KLF4 increases TNFo-induced VCAM and tissue factor (TF) expression, resulting in a pro-adhesive and pro-thrombotic inflammatory phenotype.

KLF4 was originally described as an epithelial-specific transcription factor (termed gutenriched Kruppel-like factor) ${ }^{142}$, where mutations in the gene were linked to cancer due to KLF4's regulation of cell-cycling and apoptosis ${ }^{143} . \mathrm{KLF}^{-/-}$mice die shortly after birth due to loss of skin barrier function, which results in dehydration ${ }^{144}$. KLF4 is transcription factor that contains $3 \mathrm{C} 2 \mathrm{H} 2$ type zinc fingers at its $\mathrm{C}$-terminus. These zinc fingers bind target DNA at the consensus sequence 5'-CNCCC-3' and the basic transcription element (BTE). In ECs, KLF4 transactivates the TM promoter and inhibits the cytokine-stimulated transactivation of the TF promoter ${ }^{103}$. Both the zinc finger region and a putative protein-interaction domain are required for transactivation of the TM promoter and for transrepression of the NF- $\mathrm{KB}$ pathway. 
The physiologic role of KLF4 in atherosclerosis has not been elucidated. In collaboration with the Jain lab, we tested the hypothesis that KLF4 promotes an atheroprotective endothelial phenotype. It is hypothesized that KLF4 overexpression in the endothelium protects against shear stress driven inflammation in vitro and lesion development in vivo.

\subsection{Hemodynamic Shear Stress Regulates EC KLF4}

The Jain lab previously showed that KLF4 is induced in HUVEC by laminar shear stress. To confirm this result in our model of hemodynamic shear stress, ECs were plated in monoculture as described in Chapter 3.2 and subjected to atheroprone, atheroprotective, or static conditions for 24 hours. As hypothesized, KLF4 mRNA expression is significantly higher in ECs exposed to atheroprotective flow versus atheroprone flow (Figure 12). Both flow types are significantly higher than static treatment, confirming the upregulation seen in laminar shear stresses as low as 2 dynes $/ \mathrm{cm}^{2}$. This result also points to the physiologic importance of studying KLF4 regulation by biomechanical stimuli, in addition to biochemical stimuli that is typically applied in static conditions.

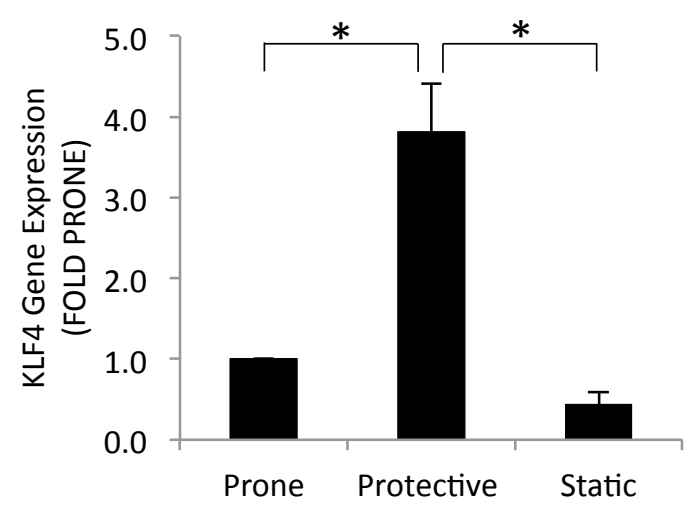

Figure 12. Hemodynamic shear stress regulates EC KLF4 (n=3). $* P<0.05$. 


\subsection{Overexpression of KLF4 Maintains an Anti-Inflammatory Phenotype}

Static in vitro studies have indicated a role for KLF4 in regulating endothelial tone and inflammation. Adenoviral overexpression of KLF4 induces TM and eNOS protein expression, and inhibits cytokine-induced PAI-1 and $\mathrm{VCAM}^{103}$. To test these responses under physiologic shear stress, ECs were plated and infected with control or KLF4 adenovirus. 24 hours after infection, ECs were exposed to atheroprone flow to test the ability of KLF4 to promote a protective phenotype.

Overexpression of KLF4 induced atheroprotective genes TM and eNOS and reduced pro-thrombotic and pro-adhesive genes PAI-1 and VCAM despite the presence of atheroprone flow (Figure 13A). Decreased VCAM protein confirmed the atheroprotective gene pattern (Figure 13B). The magnitude of the AdKLF4 results is intriguing given the normally robust inflammatory pathway demonstrated by our lab using this same monoculture atheroprone model.

In vivo mouse studies done by the Jain lab confirm the atheroprotective effects of overexpressing $\mathrm{KLF}^{145}{ }^{14}$. They generated transgenic mice expressing human KLF4 under control of the VE-cadherin promoter, on an $\mathrm{ApoE}^{-/-}$background. After 16 weeks on a high-fat diet, KLF4 Tg mice had a significantly decreased lesion burden, as measured with Sudan IV staining (Figure 13C-D). 
A

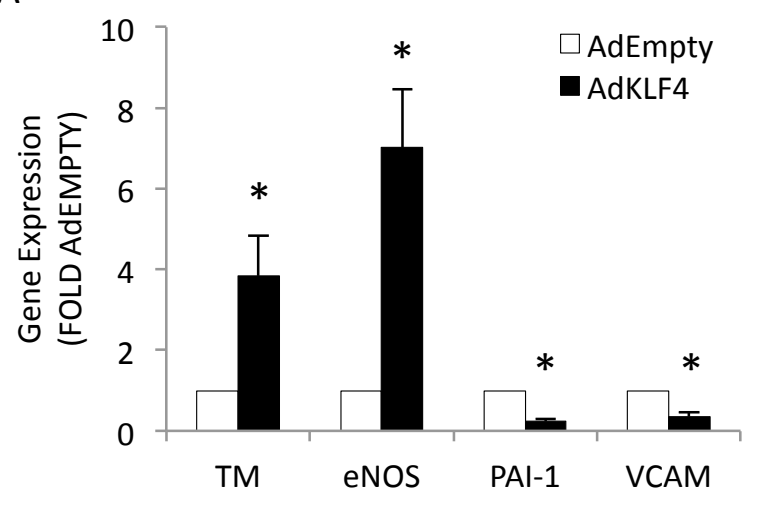

C

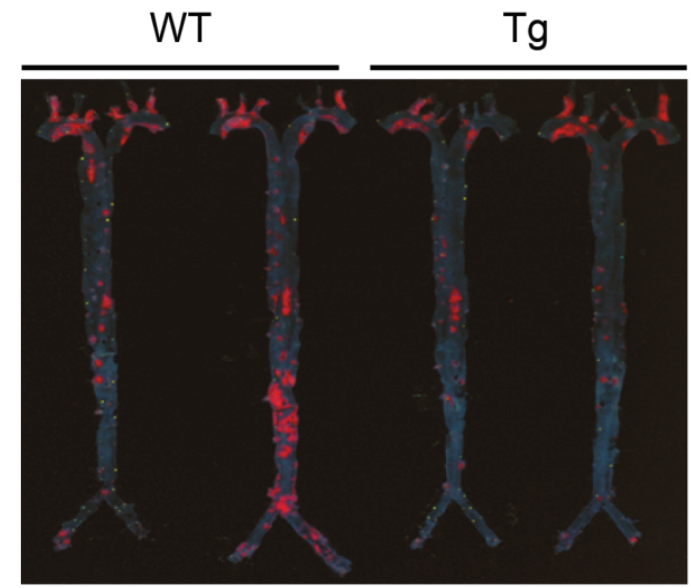

B
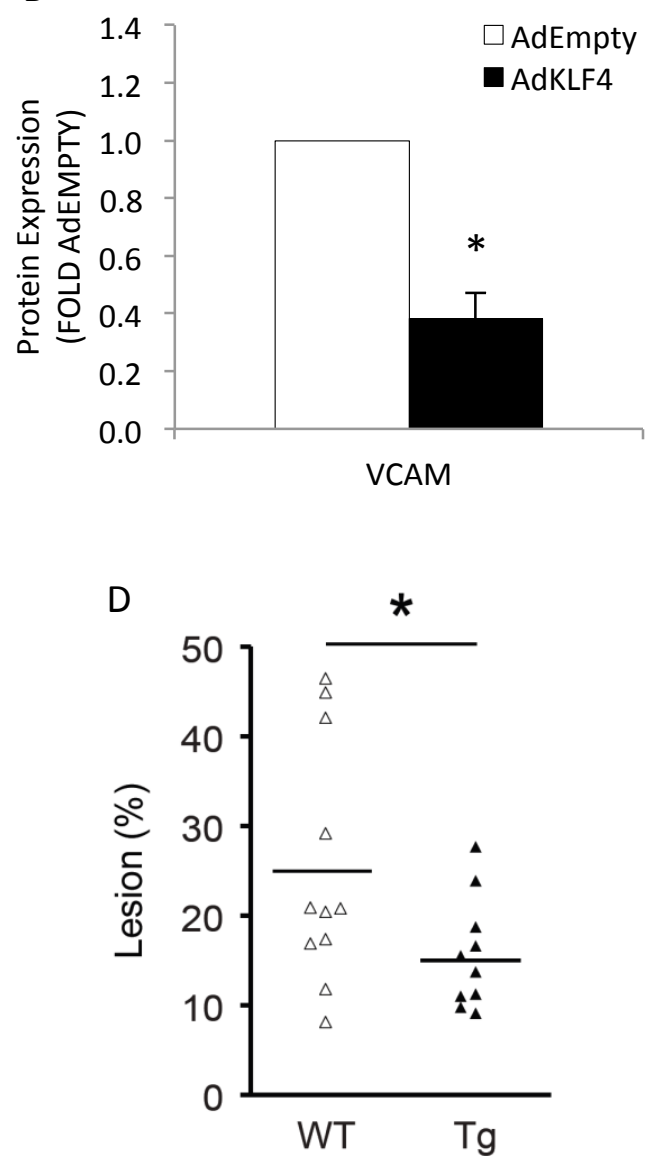

Figure 13. Overexpression of KLF4 maintains an anti-inflammatory phenotype. (A) Upregulation of vascular tone regulators (TM, eNOS) and downregulation of pro-thrombotic (PAI-1) and pro-adhesive (VCAM) genes under atheroprone flow ( $\mathrm{n}=6$ ). (B) VCAM protein decrease due to overexpression of KLF4 $(n=5)$. (C and D) EC-KLF4 Tg mice and nontransgenic WT controls on an ApoE ${ }^{-/-}$background, using the human KLF4 coding sequence under control of the VE-cadherin promoter. (C) Sudan IV staining of the entire aorta. (D) Atherosclerotic lesion area. ${ }^{*} P<0.05$. Parts C-D published in Zhou et al., 2012.

\subsection{Loss of KLF4 Promotes an Inflammatory Phenotype}

Knockdown of KLF4 by siRNA was previously shown to increase cytokine-induced PAI-1 and VCAM ${ }^{103}$. To test these responses under physiologic shear stress, ECs were plated and transfected with control or KLF4 siRNA 24 hours before exposure to flow. ECs were exposed to atheroprotective flow to test whether ECs could maintain an anti-inflammatory phenotype when KLF4 was depleted. Knockdown of KLF4 resulted in an atheroprone phenotype, despite EC exposure to atheroprotective shear stress (Figure 14A). Depletion of 
KLF4 resulted in an inflammatory EC phenotype with increased PAI-1 and VCAM, and decreased TM and eNOS. Western blotting for VCAM was unsuccessful because of the extremely low levels of expression under atheroprotective flow (data not shown).

Similarly, in vivo mouse studies done by the Jain lab confirm the necessity for KLF4 in maintaining atheroprotective effects of overexpressing KLF4 ${ }^{145}$. Floxed KLF4xVE-cadherindriven Cre mice $\left(\mathrm{KLF}^{-/-}\right)$on an $\mathrm{ApoE}^{-/-}$background were given a high fat diet for 20 weeks. $\mathrm{KLF}^{-/-}$mice had a significantly increased atherosclerotic lesion burden compared to $\mathrm{ApoE}^{-/-}$ controls (Figure 14B-C). Sudan IV staining appears increased in both atheroprone (lesser curvature of the aortic arch) and atheroprotective (abdominal aorta) regions (Figure 14B). 


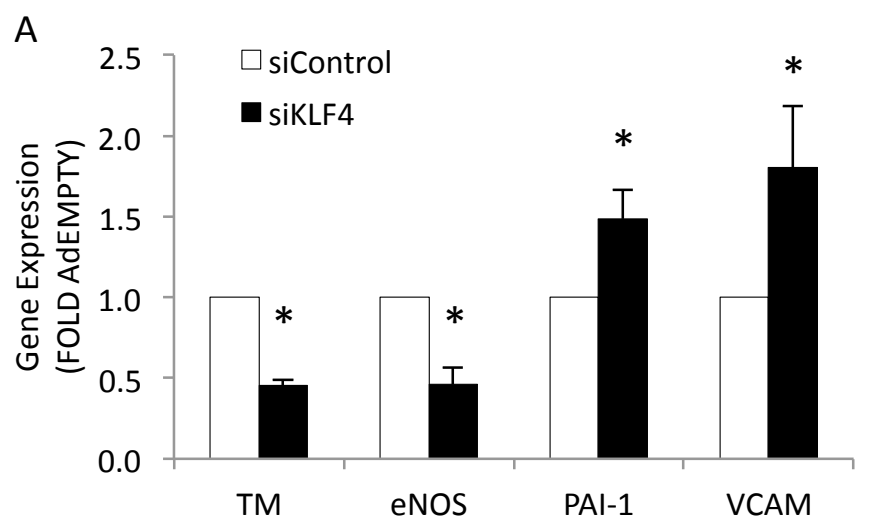

B

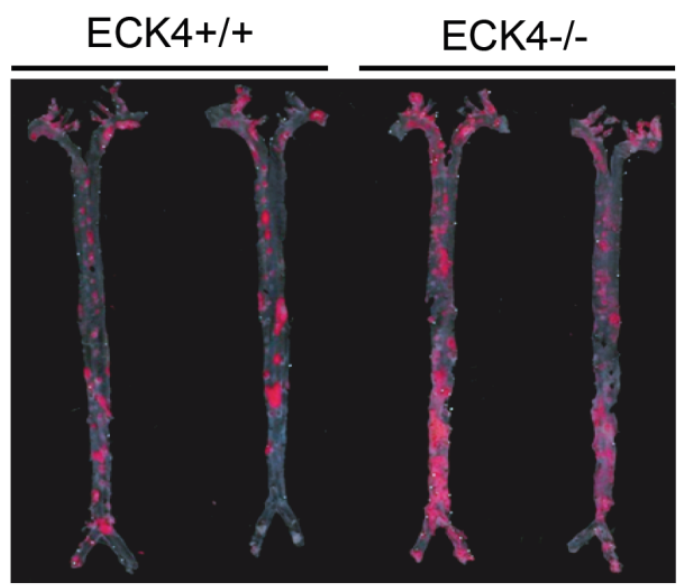

C

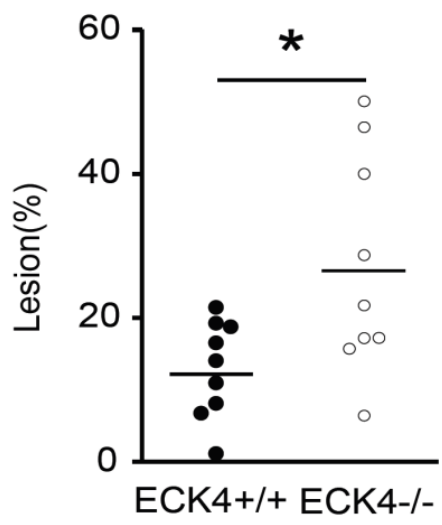

Figure 14. Knockdown of KLF4 leads to inflammatory phenotype. (A) Downregulation of vascular tone regulators (TM, eNOS) and upregulation of pro-thrombotic (PAI-1) and pro-adhesive (VCAM) genes under atheroprotective flow $(\mathrm{n}=8)$. B and C) EC KLF4-/- mice and $\mathrm{KLF} 4+/+$ controls on an $\mathrm{ApoE}^{-/-}$background. (B) Sudan IV staining of the entire aorta. (C) Atherosclerotic lesion area. ${ }^{*} P<0.05$. Parts B-C published in Zhou et al., 2012.

\subsection{Discussion}

The endothelium coordinates vascular homeostasis in health and disease. Since this vascular control is critically important, there is great interest in understanding regulators of EC gene expression. Previous work has pointed to the atheroprotective role of KLF4 in response to steady shear stress and cytokine treatment, and the current work brings that atheroprotective role into physiologic relevance. We show that KLF4 expression in ECs is upregulated in 
response to atheroprotective flow compared to atheroprone. Overexpression of KLF4 inhibits inflammatory and thrombotic genes (VCAM and PAI-1) while upregulating anti-inflammatory and anti-thrombotic genes (eNOS and TM) in vitro, and this inhibition of inflammation is functionally important in decreasing atherosclerotic lesion size in vivo. The importance of antiinflammatory KLF4 signaling is reiterated under atheroprotective flow, where loss of KLF4 leads to a loss of protective phenotype despite that hemodynamic environment. Results of both the overexpression and knockdown experiments agree in support of the hypothesis that KLF4 establishes an atheroprotective phenotype in the endothelium.

The observed KLF4-dependent changes to gene expression have a variety of potential functional consequences. In addition to its endogenous anticoagulant properties, TM protects against inflammation by interfering with cytokine-stimulated leukocyte adhesion to $\mathrm{ECs}^{103}$. ENOS mediates basal vasodilation, causing mice deficient in eNOS to be hypertensive ${ }^{146}$ and to have increased atherosclerotic lesion size ${ }^{147}$. VCAM expression is important in the recruitment of inflammatory cells during early stages of atherosclerosis, and is inhibited by overexpressing KLF4. PAI-1 may also be involved in early vascular inflammation due to its inhibition of fibrinolysis. Increased PAI-1 expression found in atherosclerotic lesions in humans promotes accumulation of fibrin and fibrinogen ${ }^{148}$. Overexpression of KLF4 in static cytokine-stimulated conditions inhibits the secretion of MCP-1 $1^{103}$, which in turn could have the functional outcome of inhibiting monocyte recruitment to the vessel wall. Overexpression of KLF4 also promotes the secretion of tissue inhibitors of matrix metalloproteinases TIMP-1 and TIMP-2, which inhibit degradation of the extracellular matrix ${ }^{103}$. By inhibiting extracellular matrix remodeling, KLF4 may be a factor in maintaining a quiescent matrix of collagen or laminin and preventing the switch to an inflammatory FN matrix. 
While the current work has focused on KLF4's regulation in ECs, it is interesting to postulate a similar regulation in SMCs. SMC KLF4 gene expression is increased under EC siFN conditions (Figure 6B), when other inflammatory markers decreased, suggesting it may be associated with atheroprotection in SMCs as well as ECs. Other research concerning KLF4 in SMCs shows conflicting results. KLF4 has increased expression in atherosclerotic lesions of $\mathrm{ApoE}^{-/-}$mice ${ }^{149}$. It also mediates phenotypic switching by binding repressor elements to inhibit SMC marker genes and by inhibiting the expression of the co-activator myocardin ${ }^{150}$. Conditional knockout of KLF4 in mice delays the repression of SM $\alpha \mathrm{A}$ and SM22 $\alpha$ that follows vascular injury $^{151}$. While these results suggest an atheroprone action of KLF4, wherein KLF4 inhibits SMC differentiation, the same knockout mice also displayed hyperproliferation and increased neointimal-thickening ${ }^{151}$. So, KLF4's conflicting roles may be tightly regulated depending on the cell environment, and may have simultaneous inflammatory and antiinflammatory consequences. KLF4 could promote atherosclerosis by inhibiting a differentiated, anti-inflammatory phenotype, while also providing the later benefit of a fibrous cap thick with SMCs that is less likely to rupture. 


\section{CHAPTER 8. SUMMARY, FUTURE DIRECTIONS, AND CLOSING REMARKS}

\subsection{Summary}

This dissertation tested the hypotheses that (1) mechanical sensing by the PECAM/FN pathway in endothelial cells regulates the smooth muscle cell inflammatory phenotype and that (2) KLF4 promotes an atheroprotective endothelial cell phenotype. Testing these hypotheses revealed the following results:

- Atheroprone hemodynamics applied to ECs increases FN deposition by ECs and cocultured SMCs compared to atheroprotective flow. This FN deposition is dependent on EC PECAM (Chapter 4).

- Atheroprone hemodynamics applied to the co-culture model induce an EC-PECAM dependent inflammatory SMC phenotype, marked by increased NF- $\mathrm{B}$ activity, VCAM, COX-2, and decreased EDA+ FN variant (Chapter 4).

- The inflammatory SMC phenotype downstream of atheroprone flow is also dependent on EC FN matrix, can be rescued with addition of exogenous FN to the EC media, and has increased SMC VCAM, COX-2, and monocyte adhesion without a change to SMC FN (Chapter 5).

- SMC FN in return promotes inflammation in SMCs and ECs via NF- $\kappa B$ activity, creating a positive feedback loop. Inhibition of SMC FN decreases the atheroprone EC phenotype without a change to EC FN (Chapter 5).

- Factors secreted during atheroprone shear stress experiments are able to induce SMC $\mathrm{NF}-\kappa \mathrm{B}$ activity in an EC PECAM and FN fashion without the physical presence of ECs (Chapter 6). 
- A milieu of at least 12 cytokines is regulated by EC FN to induce the inflammatory SMC phenotype. GRO- $\alpha$ was found capable of increasing SMC NF- $\kappa B$ activity in a dose-dependent manner on a FN matrix (Chapter 6).

- KLF4 is upregulated by atheroprotective hemodynamics in vitro compared to atheroprone or static conditions (Chapter 7).

- KLF4 overexpression rescues cells from atheroprone hemodynamics with an increase in protective genes eNOS and TM and a decrease in thrombotic and adhesive genes PAI-1 and VCAM (Chapter 7).

- Loss of KLF4 results in a loss of atheroprotection despite exposure to atheroprotective shear stress (Chapter 7).

\subsection{Future Directions}

The identification of an EC PECAM/FN/cytokine pathway inducing SMC inflammation under atheroprone hemodynamics opens up many questions to be answered with future research. SMC siFN experiments brought up an interesting mechanism for SMC-EC inflammatory feedback. Could knockdown of FN in both EC and SMCs simultaneously further inhibit inflammation in the system? If FN in either cell type signals inflammation to the other, a double knockdown approach should cause even greater reduction in NF- $\mathrm{KB}$ activity, confirming the importance of FN in promoting atherosclerosis. In an attempt to determine whether separate EC and SMC FN pools are formed in our system, we used (theoretically) specific bovine and human FN antibodies to immunostain co-cultured BAECs and HUVSMCs. The antibodies were not specific enough to determine if FN secreted by either cell type was incorporated into the matrix on the other side of the membrane. This is still a useful question to answer though, if 
higher-specificity antibodies can be developed, because the FN matrix is likely more homogenous in vivo. If SMC FN is incorporated into the EC matrix, but the EC matrix remains constant during SMC siFN, the ECs may be downregulating the MMPs involved in a protective matrix turnover while still exhibiting an overall protective phenotype.

More information can also be discovered about FN's signaling mechanism within our co-culture system. While we observe increases in SMC FN in coordination with increases in SMC NF- $\kappa B$ activity, we do not know if NF- $\kappa B$ activity is responsible for increased FN deposition. We hypothesize that NF- $\mathrm{KB}$ activity drives an increase in FN deposition, which

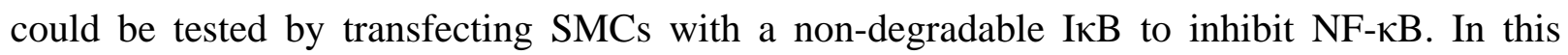
experiment, inhibition of NF- $\mathrm{kB}$ activity is predicted to reduce the atheroprone flow-induce SMC FN deposition. Another possibility is that FN in SMCs is upregulated by another

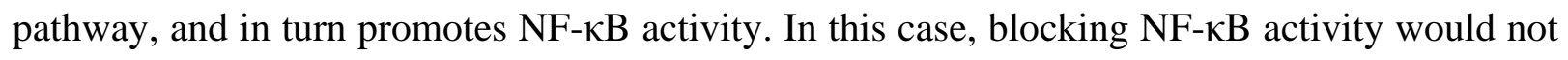
affect the amount of FN deposition. NF- $\mathrm{kB}$ activity could then be further promoted by the addition of exogenous FN to the SMC media during an atheroprone shear stress experiment.

The role of FN during atherogenesis is also untested in vivo. While FN is observed to have increased expression in atheroprone regions of the vasculature, what is its role there? FNnull mice die at embryonic day $8.5^{57}$, but mice with Cre-loxP conditional knockout of EC FN mice (driven by VE-Cadherin) on an $\mathrm{ApoE}^{-/}$background could be used to study the role of EC FN in atherogenesis. We hypothesize that EC FN plays an active role in inflammation and lesion development. A decrease in lesion size, SMC recruitment, and EC VCAM in atheroprone regions of the vasculature of EC FN knockout mice would support this hypothesis. Similarly, floxed FNxSM22-driven Cre mice could be used to study the role of SMC FN in 
providing further EC inflammation. In these mice, we would hypothesize a decrease in VCAM surrounding both SMCs and ECs in addition to decreased lesion size in atheroprone regions.

It is also interesting to postulate the role of PECAM-dependent SMC phenotype changes in vivo. PECAM mechanotransduction of shear stress occurs upstream of FN in our pathway, and exerts its influence over many proteins in addition to FN. While PECAM has a clear role in the development of atherosclerosis in ECs, its role in SMC phenotypic modulation has not been studied. The SMC phenotype mediator PDGF-DD is preferentially expressed in regions of atheroprone shear stress in vivo and in vitro, and inhibits markers of the SMC contractile phenotype ${ }^{152}$. Arterioles of PECAM knockout mice exposed to high shear stress have reduced dilation compared to WT, suggesting a potential role for PECAM's modulation of SMC vasomotor tone via $\mathrm{NO}^{153}$. More related to atheroprone signaling, PECAM deficiency in a partial carotid artery ligation model had impaired vascular remodeling and reduced intimamedia thickening ${ }^{154}$. Their research quantified vascular cell proliferation across the vessel wall, but not in SMCs specifically. If in vivo models behave as predicted from our co-culture work, $\mathrm{PECAM}^{-/} \mathrm{ApoE}^{-/-}$mice should have decreased VCAM and p65 NF- $\mathrm{kB}$ staining surrounding cells characterized by SM $\alpha$ A in atheroprone regions compared to WT. SMCs should also exhibit decreased proliferation in $\mathrm{PECAM}^{-/} \mathrm{ApoE}^{-/-}$mice compared to WT.

Our cytokine screen has revealed novel hemodynamically regulated cytokines. Some have known roles in signaling other cell types to the developing atherosclerotic lesion, such as MCP-1 recruitment of macrophages ${ }^{155}$. Others are known to stimulate SMC phenotypic switching, such as PDGF-AA's stimulation of proliferation ${ }^{102}$. What is the role of FN-regulated cytokines in promoting atherosclerosis? Additional experiments with cytokine treatment of SMCs could answer which cytokines are able to induce NF-kB activity, either alone or in 
permutations. Further work with GRO- $\alpha$ could provide details about its potential mechanism of action. Inhibition of CXCR2 (the GRO- $\alpha$ receptor, expressed on SMCs) during a co-culture experiment would determine whether GRO- $\alpha$ signaling alone induces a SMC phenotype change, or whether a more complex combination of cytokines is required. Another interesting question to investigate is how the EC cytokines are differentially regulated under flow. We hypothesize that these cytokines are under regulation by NF- $\mathrm{kB}$, as are many of the same cytokines expressed by SMCs in co-culture ${ }^{84}$. Inhibition of EC NF-kB activity through

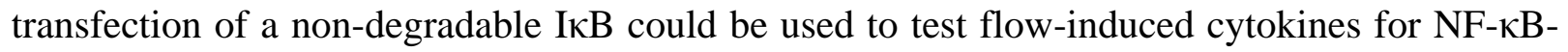
dependence. Finally, SMC FN-mediated EC inflammation pathways present another area of study, and may be cytokine-dependent as well. Is SMC-EC inflammatory signaling regulated by secreted factors/cytokines, and are those factors dependent on NF- $\kappa B$ activity?

Secreted cytokines provide a compelling EC-SMC signaling mechanism. However, other secreted factors in the media may further enhance SMC inflammation under atheroprone flow. Secreted FN could be another secreted signaling protein, due to its expression in our system and ability to promote inflammation. Is FN protein secreted into the cell media in addition to the matrix-incorporated FN protein that we measure under atheroprone shear stress? Testing media levels of FN with a FN ELISA kit would answer the question of whether FN secretion into the media is flow-dependent. MiR-221 and miR-222 have also been proposed as another signaling mechanism due to their expression by $\mathrm{ECs}^{138}$ and ability to increase SMC growth and proliferation ${ }^{137}$. MiR-143/145 is shear stress-induced in $\mathrm{ECs}^{92}$, but other miRNAs are potentially regulated by hemodynamics. MiRNA PCR arrays are now available, so screening for secreted (and flow-regulated) miRNA is possible and could present a variety of EC-SMC signaling targets. 


\subsection{Closing Remarks}

The field of vascular biology encompasses a diverse range of functions, from blood and nutrient transport to tissue development and reorganization. Along with diverse functions, the involvement of multiple cell types creates a vascular environment that is continuously responding to the integration of an large number of signaling inputs. The vascular environment is not only influenced by molecular signaling pathways but also by biomechanics, inflammation, and lipid metabolism. Perturbations to vascular signaling pathways result in diseases such as diabetes, stroke, hypertension, and atherosclerosis. As such, vascular biology research presents a critical opportunity for integrative biomedical research.

Our in vitro co-culture model represents an attempt to parse out specific cellular and molecular interactions involved in the development of atherosclerosis. Applying atheroprone hemodynamic shear stress primes the model's ECs and SMCs to adapt an activated, inflamed state. We discovered that the EC mechanosensor protein PECAM is required for the switch to an inflammatory SMC phenotype. PECAM-dependent FN matrix deposition further promotes inflammatory NF- $\kappa \mathrm{B}$ activity in both cell types. Most excitingly, our model allows us to study the method by which ECs signal an inflammatory phenotype to SMCs. We discovered that ECs secrete an intricate array of cytokines that are differentially regulated by their FN matrix. These cytokines are able to confer an inflammatory phenotype to SMCs without EC flow-regulated stimulation.

In contrast to atheroprone hemodynamics, atheroprotective hemodynamics impart an anti-inflammatory phenotype to ECs. The atheroprotective EC phenotype is measurably regulated by KLF4, which promotes transcription of an anti-inflammatory and anti-thrombotic genotype. Thus, the atheroprotective environment does not simply remove atheroprone 
stimulation, but actively signals a distinct phenotype. In attempting to therapeutically treat atherosclerosis, stimulation of protective signaling pathways may be equally important to prohibiting atheroprone pathways.

Thomas Jefferson, Founding Father and leader in the Enlightenment wrote, “ ... he who knows most, knows best how little he knows." Indeed, the deeper we delve into hemodynamic signaling mechanisms of atherosclerosis, the more questions we uncover. It is only by continuing to answer these questions that we will begin to understand the complexities within the vascular environment and be able to develop effective and specific therapeutic treatments. 


\section{CHAPTER 9. REFERENCES}

1. Libby P, Ridker PM, Hansson GK. Progress and challenges in translating the biology of atherosclerosis. Nature. 2011;473(7347):317-325.

2. Lusis AJ. Atherosclerosis. Nature. 2000;407(6801):233-241.

3. Furchgott RF, Zawadzki JV. The obligatory role of endothelial cells in the relaxation of arterial smooth muscle by acetylcholine. Nature. 1980;288(5789):373-376.

4. Cines DB, Pollak ES, Buck CA, Loscalzo J, Zimmerman GA, McEver RP, Pober JS, Wick TM, Konkle BA, Schwartz BS, Barnathan ES, McCrae KR, Hug BA, Schmidt AM, Stern DM. Endothelial cells in physiology and in the pathophysiology of vascular disorders. Blood. 1998;91(10):3527-3561.

5. Muller WA, Randolph GJ. Migration of leukocytes across endothelium and beyond: molecules involved in the transmigration and fate of monocytes. J Leukoc Biol. 1999;66(5):698-704.

6. Aird WC. Phenotypic heterogeneity of the endothelium: I. Structure, function, and mechanisms. Circ Res. 2007;100(2):158-173.

7. Dai G, Kaazempur-Mofrad MR, Natarajan S, Zhang Y, Vaughn S, Blackman BR, Kamm RD, Garcia-Cardena G, Gimbrone MA, Jr. Distinct endothelial phenotypes evoked by arterial waveforms derived from atherosclerosis-susceptible and -resistant regions of human vasculature. Proc Natl Acad Sci U S A. 2004;101(41):14871-14876.

8. Garcia-Cardena G, Comander J, Anderson KR, Blackman BR, Gimbrone MA, Jr. Biomechanical activation of vascular endothelium as a determinant of its functional phenotype. Proc Natl Acad Sci U S A. 2001;98(8):4478-4485.

9. Malek AM, Alper SL, Izumo S. Hemodynamic shear stress and its role in atherosclerosis. JAMA. 1999;282(21):2035-2042.

10. Kaazempur-Mofrad MR, Isasi AG, Younis HF, Chan RC, Hinton DP, Sukhova G, LaMuraglia GM, Lee RT, Kamm RD. Characterization of the atherosclerotic carotid bifurcation using MRI, finite element modeling, and histology. Ann Biomed Eng. 2004;32(7):932-946.

11. Gelfand BD, Epstein FH, Blackman BR. Spatial and spectral heterogeneity of timevarying shear stress profiles in the carotid bifurcation by phase-contrast MRI. J Magn Reson Imaging. 2006;24(6):1386-1392.

12. Feaver RE, Gelfand BD, Blackman BR. Human haemodynamic frequency harmonics regulate the inflammatory phenotype of vascular endothelial cells. Nat Commun. 2013;4:1525.

13. Atkins GB, Wang Y, Mahabeleshwar GH, Shi H, Gao H, Kawanami D, Natesan V, Lin Z, Simon DI, Jain MK. Hemizygous deficiency of Kruppel-like factor 2 augments experimental atherosclerosis. Circ Res. 2008;103(7):690-693.

14. Dekker RJ, van Soest S, Fontijn RD, Salamanca S, de Groot PG, VanBavel E, Pannekoek H, Horrevoets AJ. Prolonged fluid shear stress induces a distinct set of endothelial cell genes, most specifically lung Kruppel-like factor (KLF2). Blood. 2002;100(5):1689-1698.

15. Dai G, Vaughn S, Zhang Y, Wang ET, Garcia-Cardena G, Gimbrone MA, Jr. Biomechanical forces in atherosclerosis-resistant vascular regions regulate endothelial redox balance via phosphoinositol 3-kinase/Akt-dependent activation of Nrf2. Circ Res. 2007;101(7):723-733. 
16. Topper JN, Cai J, Falb D, Gimbrone MA, Jr. Identification of vascular endothelial genes differentially responsive to fluid mechanical stimuli: cyclooxygenase-2, manganese superoxide dismutase, and endothelial cell nitric oxide synthase are selectively up-regulated by steady laminar shear stress. Proc Natl Acad Sci U S A. 1996;93(19):10417-10422.

17. Gelfand BD, Meller J, Pryor AW, Kahn M, Bortz PD, Wamhoff BR, Blackman BR. Hemodynamic activation of beta-catenin and T-cell-specific transcription factor signaling in vascular endothelium regulates fibronectin expression. Arterioscler Thromb Vasc Biol. 2011;31(7):1625-1633.

18. Chappell DC, Varner SE, Nerem RM, Medford RM, Alexander RW. Oscillatory shear stress stimulates adhesion molecule expression in cultured human endothelium. Circ Res. 1998;82(5):532-539.

19. Iiyama K, Hajra L, Iiyama M, Li H, DiChiara M, Medoff BD, Cybulsky MI. Patterns of vascular cell adhesion molecule-1 and intercellular adhesion molecule-1 expression in rabbit and mouse atherosclerotic lesions and at sites predisposed to lesion formation. Circ Res. 1999;85(2):199-207.

20. Nagel T, Resnick N, Atkinson WJ, Dewey CF, Jr., Gimbrone MA, Jr. Shear stress selectively upregulates intercellular adhesion molecule-1 expression in cultured human vascular endothelial cells. J Clin Invest. 1994;94(2):885-891.

21. Dinarello CA. Historical insights into cytokines. Eur J Immunol. 2007;37 Suppl 1:S3445.

22. Hagiwara H, Mitsumata M, Yamane T, Jin X, Yoshida Y. Laminar shear stress-induced GRO mRNA and protein expression in endothelial cells. Circulation. 1998;98(23):2584-2590.

23. Burke-Gaffney A, Brooks AV, Bogle RG. Regulation of chemokine expression in atherosclerosis. Vascul Pharmacol. 2002;38(5):283-292.

24. Conklin BS, Zhong DS, Zhao W, Lin PH, Chen C. Shear stress regulates occludin and VEGF expression in porcine arterial endothelial cells. J Surg Res. 2002;102(1):13-21.

25. Chang K, Weiss D, Suo J, Vega JD, Giddens D, Taylor WR, Jo H. Bone morphogenic protein antagonists are coexpressed with bone morphogenic protein 4 in endothelial cells exposed to unstable flow in vitro in mouse aortas and in human coronary arteries: role of bone morphogenic protein antagonists in inflammation and atherosclerosis. Circulation. 2007;116(11):1258-1266.

26. Burns MP, DePaola N. Flow-conditioned HUVECs support clustered leukocyte adhesion by coexpressing ICAM-1 and E-selectin. Am J Physiol Heart Circ Physiol. 2005;288(1):H194-204.

27. Cicha I, Beronov K, Ramirez EL, Osterode K, Goppelt-Struebe M, Raaz D, Yilmaz A, Daniel WG, Garlichs CD. Shear stress preconditioning modulates endothelial susceptibility to circulating TNF-alpha and monocytic cell recruitment in a simplified model of arterial bifurcations. Atherosclerosis. 2009;207(1):93-102.

28. Gonzales RS, Wick TM. Hemodynamic modulation of monocytic cell adherence to vascular endothelium. Ann Biomed Eng. 1996;24(3):382-393.

29. Dardik A, Chen L, Frattini J, Asada H, Aziz F, Kudo FA, Sumpio BE. Differential effects of orbital and laminar shear stress on endothelial cells. J Vasc Surg. 2005;41(5):869-880. 
30. Mattart M, Mazzolai L, Chambaz C, Hayoz D, Brunner HR, Silacci P. ET-1 and NOS III gene expression regulation by plaque-free and plaque-prone hemodynamic conditions. Biorheology. 2003;40(1-3):289-297.

31. Dancu MB, Berardi DE, Vanden Heuvel JP, Tarbell JM. Asynchronous shear stress and circumferential strain reduces endothelial NO synthase and cyclooxygenase-2 but induces endothelin-1 gene expression in endothelial cells. Arterioscler Thromb Vasc Biol. 2004;24(11):2088-2094.

32. Jo H, Song H, Mowbray A. Role of NADPH oxidases in disturbed flow- and BMP4induced inflammation and atherosclerosis. Antioxid Redox Signal. 2006;8(9-10):16091619.

33. Orr AW, Stockton R, Simmers MB, Sanders JM, Sarembock IJ, Blackman BR, Schwartz MA. Matrix-specific p21-activated kinase activation regulates vascular permeability in atherogenesis. J Cell Biol. 2007;176(5):719-727.

34. Himburg HA, Grzybowski DM, Hazel AL, LaMack JA, Li XM, Friedman MH. Spatial comparison between wall shear stress measures and porcine arterial endothelial permeability. Am J Physiol Heart Circ Physiol. 2004;286(5):H1916-1922.

35. Cunningham KS, Gotlieb AI. The role of shear stress in the pathogenesis of atherosclerosis. Lab Invest. 2005;85(1):9-23.

36. Hahn C, Schwartz MA. The role of cellular adaptation to mechanical forces in atherosclerosis. Arterioscler Thromb Vasc Biol. 2008;28(12):2101-2107.

37. Tzima E, Irani-Tehrani M, Kiosses WB, Dejana E, Schultz DA, Engelhardt B, Cao G, DeLisser H, Schwartz MA. A mechanosensory complex that mediates the endothelial cell response to fluid shear stress. Nature. 2005;437(7057):426-431.

38. Chen KD, Li YS, Kim M, Li S, Yuan S, Chien S, Shyy JY. Mechanotransduction in response to shear stress. Roles of receptor tyrosine kinases, integrins, and Shc. J Biol Chem. 1999;274(26):18393-18400.

39. Hajra L, Evans AI, Chen M, Hyduk SJ, Collins T, Cybulsky MI. The NF-kappa B signal transduction pathway in aortic endothelial cells is primed for activation in regions predisposed to atherosclerotic lesion formation. Proc Natl Acad Sci $U$ S A. 2000;97(16):9052-9057.

40. Chiu YJ, McBeath E, Fujiwara K. Mechanotransduction in an extracted cell model: Fyn drives stretch- and flow-elicited PECAM-1 phosphorylation. $J$ Cell Biol. 2008;182(4):753-763.

41. Osawa M, Masuda M, Harada N, Lopes RB, Fujiwara K. Tyrosine phosphorylation of platelet endothelial cell adhesion molecule-1 (PECAM-1, CD31) in mechanically stimulated vascular endothelial cells. Eur J Cell Biol. 1997;72(3):229-237.

42. Fujiwara $\mathrm{K}$, Masuda $\mathrm{M}$, Osawa $\mathrm{M}$, Kano $\mathrm{Y}$, Katoh K. Is PECAM-1 a mechanoresponsive molecule? Cell Struct Funct. 2001;26(1):11-17.

43. Jackson DE, Ward CM, Wang R, Newman PJ. The protein-tyrosine phosphatase SHP-2 binds platelet/endothelial cell adhesion molecule-1 (PECAM-1) and forms a distinct signaling complex during platelet aggregation. Evidence for a mechanistic link between PECAM-1- and integrin-mediated cellular signaling. J Biol Chem. 1997;272(11):69866993.

44. Masuda M, Osawa M, Shigematsu H, Harada N, Fujiwara K. Platelet endothelial cell adhesion molecule-1 is a major SH-PTP2 binding protein in vascular endothelial cells. FEBS Lett. 1997;408(3):331-336. 
45. Osawa M, Masuda M, Kusano K, Fujiwara K. Evidence for a role of platelet endothelial cell adhesion molecule-1 in endothelial cell mechanosignal transduction: is it a mechanoresponsive molecule? J Cell Biol. 2002;158(4):773-785.

46. Tai LK, Zheng Q, Pan S, Jin ZG, Berk BC. Flow activates ERK1/2 and endothelial nitric oxide synthase via a pathway involving PECAM1, SHP2, and Tie2. J Biol Chem. 2005;280(33):29620-29624.

47. Fleming I, Fisslthaler B, Dixit M, Busse R. Role of PECAM-1 in the shear-stressinduced activation of Akt and the endothelial nitric oxide synthase (eNOS) in endothelial cells. J Cell Sci. 2005;118(Pt 18):4103-4111.

48. Feaver RE, Gelfand BD, Wang C, Schwartz MA, Blackman BR. Atheroprone hemodynamics regulate fibronectin deposition to create positive feedback that sustains endothelial inflammation. Circ Res. 2010;106(11):1703-1711.

49. Collins C, Guilluy C, Welch C, O'Brien ET, Hahn K, Superfine R, Burridge K, Tzima E. Localized tensional forces on PECAM-1 elicit a global mechanotransduction response via the integrin-RhoA pathway. Curr Biol. 2012;22(22):2087-2094.

50. Nakashima Y, Plump AS, Raines EW, Breslow JL, Ross R. ApoE-deficient mice develop lesions of all phases of atherosclerosis throughout the arterial tree. Arterioscler Thromb. 1994;14(1):133-140.

51. Harry BL, Sanders JM, Feaver RE, Lansey M, Deem TL, Zarbock A, Bruce AC, Pryor AW, Gelfand BD, Blackman BR, Schwartz MA, Ley K. Endothelial cell PECAM-1 promotes atherosclerotic lesions in areas of disturbed flow in ApoE-deficient mice. Arterioscler Thromb Vasc Biol. 2008;28(11):2003-2008.

52. Stevens HY, Melchior B, Bell KS, Yun S, Yeh JC, Frangos JA. PECAM-1 is a critical mediator of atherosclerosis. Dis Model Mech. 2008;1(2-3):175-181; discussion 179.

53. Goel R, Schrank BR, Arora S, Boylan B, Fleming B, Miura H, Newman PJ, Molthen RC, Newman DK. Site-specific effects of PECAM-1 on atherosclerosis in LDL receptor-deficient mice. Arterioscler Thromb Vasc Biol. 2008;28(11):1996-2002.

54. ffrench-Constant C. Alternative splicing of fibronectin--many different proteins but few different functions. Exp Cell Res. 1995;221(2):261-271.

55. Pankov R, Yamada KM. Fibronectin at a glance. J Cell Sci. 2002;115(Pt 20):38613863.

56. Guan JL, Trevithick JE, Hynes RO. Retroviral expression of alternatively spliced forms of rat fibronectin. J Cell Biol. 1990;110(3):833-847.

57. George EL, Georges-Labouesse EN, Patel-King RS, Rayburn H, Hynes RO. Defects in mesoderm, neural tube and vascular development in mouse embryos lacking fibronectin. Development. 1993;119(4):1079-1091.

58. Chiang HY, Korshunov VA, Serour A, Shi F, Sottile J. Fibronectin is an important regulator of flow-induced vascular remodeling. Arterioscler Thromb Vasc Biol. 2009;29(7):1074-1079.

59. Sun Z, Martinez-Lemus LA, Hill MA, Meininger GA. Extracellular matrix-specific focal adhesions in vascular smooth muscle produce mechanically active adhesion sites. Am J Physiol Cell Physiol. 2008;295(1):C268-278.

60. Pedretti M, Rancic Z, Soltermann A, Herzog BA, Schliemann C, Lachat M, Neri D, Kaufmann PA. Comparative immunohistochemical staining of atherosclerotic plaques using F16, F8 and L19: Three clinical-grade fully human antibodies. Atherosclerosis. 2009. 
61. Stenman S, von Smitten K, Vaheri A. Fibronectin and atherosclerosis. Acta Med Scand Suppl. 1980;642:165-170.

62. Labat-Robert J, Szendroi M, Godeau G, Robert L. Comparative distribution patterns of type I and III collagens and fibronectin in human arteriosclerotic aorta. Pathol Biol (Paris). 1985;33(4):261-265.

63. Shekhonin BV, Domogatsky SP, Idelson GL, Koteliansky VE, Rukosuev VS. Relative distribution of fibronectin and type I, III, IV, V collagens in normal and atherosclerotic intima of human arteries. Atherosclerosis. 1987;67(1):9-16.

64. Orr AW, Sanders JM, Bevard M, Coleman E, Sarembock IJ, Schwartz MA. The subendothelial extracellular matrix modulates NF-kappaB activation by flow: a potential role in atherosclerosis. J Cell Biol. 2005;169(1):191-202.

65. Tanouchi J, Uematsu M, Kitabatake A, Masuyama T, Ito H, Doi Y, Inoue M, Kamada T. Sequential appearance of fibronectin, collagen and elastin during fatty streak initiation and maturation in hypercholesterolemic fat-fed rabbits. Jpn Circ J. 1992;56(7):649-656.

66. Hahn C, Orr AW, Sanders JM, Jhaveri KA, Schwartz MA. The subendothelial extracellular matrix modulates JNK activation by flow. Circ Res. 2009;104(8):9951003.

67. Orr AW, Hahn C, Blackman BR, Schwartz MA. p21-activated kinase signaling regulates oxidant-dependent NF-kappa B activation by flow. Circ Res. 2008;103(6):671-679.

68. Owens GK. Regulation of differentiation of vascular smooth muscle cells. Physiol Rev. 1995;75(3):487-517.

69. Owens GK, Kumar MS, Wamhoff BR. Molecular regulation of vascular smooth muscle cell differentiation in development and disease. Physiol Rev. 2004;84(3):767-801.

70. Orr AW, Hastings NE, Blackman BR, Wamhoff BR. Complex Regulation and Function of the Inflammatory Smooth Muscle Cell Phenotype in Atherosclerosis. J Vasc Res. 2009;47(2):168-180.

71. Brand K, Page S, Rogler G, Bartsch A, Brandl R, Knuechel R, Page M, Kaltschmidt C, Baeuerle PA, Neumeier D. Activated transcription factor nuclear factor-kappa B is present in the atherosclerotic lesion. J Clin Invest. 1996;97(7):1715-1722.

72. Hastings NE, Feaver RE, Lee MY, Wamhoff BR, Blackman BR. Human IL-8 regulates smooth muscle cell VCAM-1 expression in response to endothelial cells exposed to atheroprone flow. Arterioscler Thromb Vasc Biol. 2009;29(5):725-731.

73. Orr AW, Lee MY, Lemmon JA, Yurdagul A, Jr., Gomez MF, Bortz PD, Wamhoff BR. Molecular mechanisms of collagen isotype-specific modulation of smooth muscle cell phenotype. Arterioscler Thromb Vasc Biol. 2009;29(2):225-231.

74. Liu Z, Dronadula N, Rao GN. A novel role for nuclear factor of activated $\mathrm{T}$ cells in receptor tyrosine kinase and $\mathrm{G}$ protein-coupled receptor agonist-induced vascular smooth muscle cell motility. J Biol Chem. 2004;279(39):41218-41226.

75. Yellaturu CR, Ghosh SK, Rao RK, Jennings LK, Hassid A, Rao GN. A potential role for nuclear factor of activated $\mathrm{T}$-cells in receptor tyrosine kinase and G-protein-coupled receptor agonist-induced cell proliferation. Biochem J. 2002;368(Pt 1):183-190.

76. Seki Y, Kai H, Shibata R, Nagata T, Yasukawa H, Yoshimura A, Imaizumi T. Role of the JAK/STAT pathway in rat carotid artery remodeling after vascular injury. Circ Res. 2000;87(1):12-18. 
77. Neeli I, Liu Z, Dronadula N, Ma ZA, Rao GN. An essential role of the Jak-2/STAT3/cytosolic phospholipase $\mathrm{A}(2)$ axis in platelet-derived growth factor BB-induced vascular smooth muscle cell motility. J Biol Chem. 2004;279(44):46122-46128.

78. Marrero MB, Schieffer B, Li B, Sun J, Harp JB, Ling BN. Role of Janus kinase/signal transducer and activator of transcription and mitogen-activated protein kinase cascades in angiotensin II- and platelet-derived growth factor-induced vascular smooth muscle cell proliferation. J Biol Chem. 1997;272(39):24684-24690.

79. Libby P, Sukhova G, Lee RT, Galis ZS. Cytokines regulate vascular functions related to stability of the atherosclerotic plaque. J Cardiovasc Pharmacol. 1995;25 Suppl 2:S9-12.

80. Molossi S, Clausell N, Rabinovitch M. Reciprocal induction of tumor necrosis factoralpha and interleukin-1 beta activity mediates fibronectin synthesis in coronary artery smooth muscle cells. J Cell Physiol. 1995;163(1):19-29.

81. Qwarnstrom EE, Ostberg CO, Turk GL, Richardson CA, Bomsztyk K. Fibronectin attachment activates the NF-kappa B p50/p65 heterodimer in fibroblasts and smooth muscle cells. J Biol Chem. 1994;269(49):30765-30768.

82. Yamamoto M, Yamamoto K, Noumura T. Type I collagen promotes modulation of cultured rabbit arterial smooth muscle cells from a contractile to a synthetic phenotype. Exp Cell Res. 1993;204(1):121-129.

83. Chiu JJ, Chen LJ, Lee PL, Lee CI, Lo LW, Usami S, Chien S. Shear stress inhibits adhesion molecule expression in vascular endothelial cells induced by coculture with smooth muscle cells. Blood. 2003;101(7):2667-2674.

84. Chiu JJ, Chen LJ, Chang SF, Lee PL, Lee CI, Tsai MC, Lee DY, Hsieh HP, Usami S, Chien S. Shear stress inhibits smooth muscle cell-induced inflammatory gene expression in endothelial cells: role of NF-kappaB. Arterioscler Thromb Vasc Biol. 2005;25(5):963-969.

85. Tsai MC, Chen L, Zhou J, Tang Z, Hsu TF, Wang Y, Shih YT, Peng HH, Wang N, Guan Y, Chien S, Chiu JJ. Shear stress induces synthetic-to-contractile phenotypic modulation in smooth muscle cells via peroxisome proliferator-activated receptor alpha/delta activations by prostacyclin released by sheared endothelial cells. Circ Res. 2009;105(5):471-480.

86. Wallace CS, Champion JC, Truskey GA. Adhesion and function of human endothelial cells co-cultured on smooth muscle cells. Ann Biomed Eng. 2007;35(3):375-386.

87. Wallace CS, Truskey GA. Direct-contact co-culture between smooth muscle and endothelial cells inhibits TNF-alpha-mediated endothelial cell activation. Am J Physiol Heart Circ Physiol. 2010;299(2):H338-346.

88. Hastings NE, Simmers MB, McDonald OG, Wamhoff BR, Blackman BR. Atherosclerosis-prone hemodynamics differentially regulates endothelial and smooth muscle cell phenotypes and promotes pro-inflammatory priming. Am J Physiol Cell Physiol. 2007;293(6):C1824-1833.

89. Gilbertson-Beadling SK, Fisher C. A potential role for N-cadherin in mediating endothelial cell-smooth muscle cell interactions in the rat vasculature. Lab Invest. 1993;69(2):203-209.

90. Rama A, Matsushita T, Charolidi N, Rothery S, Dupont E, Severs NJ. Up-regulation of connexin43 correlates with increased synthetic activity and enhanced contractile differentiation in TGF-beta-treated human aortic smooth muscle cells. Eur J Cell Biol. 2006;85(5):375-386. 
91. Matthys KE, Bult H. Nitric oxide function in atherosclerosis. Mediators Inflamm. 1997;6(1):3-21.

92. Hergenreider E, Heydt S, Treguer K, Boettger T, Horrevoets AJ, Zeiher AM, Scheffer MP, Frangakis AS, Yin X, Mayr M, Braun T, Urbich C, Boon RA, Dimmeler S. Atheroprotective communication between endothelial cells and smooth muscle cells through miRNAs. Nat Cell Biol. 2012;14(3):249-256.

93. Katsuda S, Kaji T. Atherosclerosis and extracellular matrix. J Atheroscler Thromb. 2003;10(5):267-274.

94. Ait-Oufella H, Taleb S, Mallat Z, Tedgui A. Recent advances on the role of cytokines in atherosclerosis. Arterioscler Thromb Vasc Biol. 2011;31(5):969-979.

95. Sterpetti AV, Cucina A, Morena AR, Di Donna S, D'Angelo LS, Cavalarro A, Stipa S. Shear stress increases the release of interleukin-1 and interleukin- 6 by aortic endothelial cells. Surgery. 1993;114(5):911-914.

96. Tang R, Zhou J, Cheng M, Nie Y, Wu J, Chen H. [Time-dependent increase of interleukin-8 production in endothelial cells exposed to fluid shear stress]. Sheng $W u Y i$ Xue Gong Cheng Xue Za Zhi. 2004;21(1):34-37.

97. Ji JY, Jing H, Diamond SL. Hemodynamic regulation of inflammation at the endothelial-neutrophil interface. Ann Biomed Eng. 2008;36(4):586-595.

98. Cheng C, Tempel D, van Haperen R, de Boer HC, Segers D, Huisman M, van Zonneveld AJ, Leenen PJ, van der Steen A, Serruys PW, de Crom R, Krams R. Shear stress-induced changes in atherosclerotic plaque composition are modulated by chemokines. J Clin Invest. 2007;117(3):616-626.

99. $\mathrm{Yu} \mathrm{H}$, Zeng Y, Hu J, Li C. Fluid shear stress induces the secretion of monocyte chemoattractant protein-1 in cultured human umbilical vein endothelial cells. Clin Hemorheol Microcirc. 2002;26(3):199-207.

100. Moehle CW, Bhamidipati CM, Alexander MR, Mehta GS, Irvine JN, Salmon M, Upchurch GR, Jr., Kron IL, Owens GK, Ailawadi G. Bone marrow-derived MCP1 required for experimental aortic aneurysm formation and smooth muscle phenotypic modulation. J Thorac Cardiovasc Surg. 2011;142(6):1567-1574.

101. Dardik A, Yamashita A, Aziz F, Asada H, Sumpio BE. Shear stress-stimulated endothelial cells induce smooth muscle cell chemotaxis via platelet-derived growth factor-BB and interleukin-1alpha. J Vasc Surg. 2005;41(2):321-331.

102. Li L, Blumenthal DK, Terry CM, He Y, Carlson ML, Cheung AK. PDGF-induced proliferation in human arterial and venous smooth muscle cells: molecular basis for differential effects of PDGF isoforms. J Cell Biochem. 2011;112(1):289-298.

103. Hamik A, Lin Z, Kumar A, Balcells M, Sinha S, Katz J, Feinberg MW, Gerzsten RE, Edelman ER, Jain MK. Kruppel-like factor 4 regulates endothelial inflammation. J Biol Chem. 2007;282(18):13769-13779.

104. McCormick SM, Eskin SG, McIntire LV, Teng CL, Lu CM, Russell CG, Chittur KK. DNA microarray reveals changes in gene expression of shear stressed human umbilical vein endothelial cells. Proc Natl Acad Sci U S A. 2001;98(16):8955-8960.

105. Blackman BR, Garcia-Cardena G, Gimbrone MA, Jr. A new in vitro model to evaluate differential responses of endothelial cells to simulated arterial shear stress waveforms. $J$ Biomech Eng. 2002;124(4):397-407.

106. Jin L, Hastings NE, Blackman BR, Somlyo AV. Mechanical properties of the extracellular matrix alter expression of smooth muscle protein LPP and its partner 
palladin; relationship to early atherosclerosis and vascular injury. J Muscle Res Cell Motil. 2009;30(1-2):41-55.

107. Liao YF, Gotwals PJ, Koteliansky VE, Sheppard D, Van De Water L. The EIIIA segment of fibronectin is a ligand for integrins alpha 9beta 1 and alpha 4beta 1 providing a novel mechanism for regulating cell adhesion by alternative splicing. $J$ Biol Chem. 2002;277(17):14467-14474.

108. Kohan M, Muro AF, White ES, Berkman N. EDA-containing cellular fibronectin induces fibroblast differentiation through binding to alpha4beta7 integrin receptor and MAPK/Erk 1/2-dependent signaling. FASEB J. 2010;24(11):4503-4512.

109. Dietrich T, Perlitz C, Licha K, Stawowy P, Atrott K, Tachezy M, Meyborg H, Stocker C, Grafe M, Fleck E, Schirner M, Graf K. ED-B fibronectin (ED-B) can be targeted using a novel single chain antibody conjugate and is associated with macrophage accumulation in atherosclerotic lesions. Basic Res Cardiol. 2007;102(4):298-307.

110. Cai Q, Lanting L, Natarajan R. Interaction of monocytes with vascular smooth muscle cells regulates monocyte survival and differentiation through distinct pathways. Arterioscler Thromb Vasc Biol. 2004;24(12):2263-2270.

111. Braun M, Pietsch P, Schror K, Baumann G, Felix SB. Cellular adhesion molecules on vascular smooth muscle cells. Cardiovasc Res. 1999;41(2):395-401.

112. Doran AC, Meller N, McNamara CA. Role of smooth muscle cells in the initiation and early progression of atherosclerosis. Arterioscler Thromb Vasc Biol. 2008;28(5):812819.

113. Alexander MR, Murgai M, Moehle CW, Owens GK. Interleukin-1beta modulates smooth muscle cell phenotype to a distinct inflammatory state relative to PDGF-DD via NF-kappaB-dependent mechanisms. Physiol Genomics. 2012;44(7):417-429.

114. Fetalvero KM, Shyu M, Nomikos AP, Chiu YF, Wagner RJ, Powell RJ, Hwa J, Martin KA. The prostacyclin receptor induces human vascular smooth muscle cell differentiation via the protein kinase A pathway. Am J Physiol Heart Circ Physiol. 2006;290(4):H1337-1346.

115. Roan JN, Tsai YC, Chen IW, Chang SW, Huang CC, Lam CF. Inhibition of cyclooxygenase-2 modulates phenotypic switching of vascular smooth muscle cells during increased aortic blood flow. Heart Vessels. 2012;27(3):307-315.

116. Kobayashi T, Tahara Y, Matsumoto M, Iguchi M, Sano H, Murayama T, Arai H, Oida H, Yurugi-Kobayashi T, Yamashita JK, Katagiri H, Majima M, Yokode M, Kita T, Narumiya S. Roles of thromboxane $\mathrm{A}(2)$ and prostacyclin in the development of atherosclerosis in apoE-deficient mice. J Clin Invest. 2004;114(6):784-794.

117. Murata T, Ushikubi F, Matsuoka T, Hirata M, Yamasaki A, Sugimoto Y, Ichikawa A, Aze Y, Tanaka T, Yoshida N, Ueno A, Oh-ishi S, Narumiya S. Altered pain perception and inflammatory response in mice lacking prostacyclin receptor. Nature. 1997;388(6643):678-682.

118. Okamura Y, Watari M, Jerud ES, Young DW, Ishizaka ST, Rose J, Chow JC, Strauss JF, 3rd. The extra domain A of fibronectin activates Toll-like receptor 4. J Biol Chem. 2001;276(13):10229-10233.

119. van Keulen JK, de Kleijn DP, Nijhuis MM, Busser E, Velema E, Fijnheer R, van der Graaf Y, Moll FL, de Vries JP, Pasterkamp G. Levels of extra domain A containing fibronectin in human atherosclerotic plaques are associated with a stable plaque phenotype. Atherosclerosis. 2007;195(1):e83-91. 
120. Babaev VR, Porro F, Linton MF, Fazio S, Baralle FE, Muro AF. Absence of regulated splicing of fibronectin EDA exon reduces atherosclerosis in mice. Atherosclerosis. 2008;197(2):534-540.

121. Autieri MV, Yue TL, Ferstein GZ, Ohlstein E. Antisense oligonucleotides to the p65 subunit of NF-kB inhibit human vascular smooth muscle cell adherence and proliferation and prevent neointima formation in rat carotid arteries. Biochem Biophys Res Commun. 1995;213(3):827-836.

122. Huo Y, Ley K. Adhesion molecules and atherogenesis. Acta Physiol Scand. 2001;173(1):35-43.

123. Tzima E, del Pozo MA, Shattil SJ, Chien S, Schwartz MA. Activation of integrins in endothelial cells by fluid shear stress mediates Rho-dependent cytoskeletal alignment. EMBO J. 2001;20(17):4639-4647.

124. Huveneers S, Truong H, Fassler R, Sonnenberg A, Danen EH. Binding of soluble fibronectin to integrin alpha5 beta1 - link to focal adhesion redistribution and contractile shape. J Cell Sci. 2008;121(Pt 15):2452-2462.

125. Conforti G, Dominguez-Jimenez C, Zanetti A, Gimbrone MA, Jr., Cremona O, Marchisio PC, Dejana E. Human endothelial cells express integrin receptors on the luminal aspect of their membrane. Blood. 1992;80(2):437-446.

126. Shi F, Sottile J. Caveolin-1-dependent beta1 integrin endocytosis is a critical regulator of fibronectin turnover. J Cell Sci. 2008;121(Pt 14):2360-2371.

127. Tzima E, Del Pozo MA, Kiosses WB, Mohamed SA, Li S, Chien S, Schwartz MA. Activation of Rac1 by shear stress in endothelial cells mediates both cytoskeletal reorganization and effects on gene expression. EMBO J. 2002;21(24):6791-6800.

128. Hedin U, Bottger BA, Forsberg E, Johansson S, Thyberg J. Diverse effects of fibronectin and laminin on phenotypic properties of cultured arterial smooth muscle cells. J Cell Biol. 1988;107(1):307-319.

129. Thyberg J, Hultgardh-Nilsson A. Fibronectin and the basement membrane components laminin and collagen type IV influence the phenotypic properties of subcultured rat aortic smooth muscle cells differently. Cell Tissue Res. 1994;276(2):263-271.

130. Hirst SJ, Twort $\mathrm{CH}$, Lee TH. Differential effects of extracellular matrix proteins on human airway smooth muscle cell proliferation and phenotype. Am J Respir Cell Mol Biol. 2000;23(3):335-344.

131. Li H, Cybulsky MI, Gimbrone MA, Jr., Libby P. An atherogenic diet rapidly induces VCAM-1, a cytokine-regulatable mononuclear leukocyte adhesion molecule, in rabbit aortic endothelium. Arterioscler Thromb. 1993;13(2):197-204.

132. Huo Y, Weber C, Forlow SB, Sperandio M, Thatte J, Mack M, Jung S, Littman DR, Ley $\mathrm{K}$. The chemokine $\mathrm{KC}$, but not monocyte chemoattractant protein-1, triggers monocyte arrest on early atherosclerotic endothelium. J Clin Invest. 2001;108(9):13071314.

133. Boisvert WA, Rose DM, Johnson KA, Fuentes ME, Lira SA, Curtiss LK, Terkeltaub RA. Up-regulated expression of the CXCR2 ligand KC/GRO-alpha in atherosclerotic lesions plays a central role in macrophage accumulation and lesion progression. Am J Pathol. 2006;168(4):1385-1395.

134. Liehn EA, Schober A, Weber C. Blockade of keratinocyte-derived chemokine inhibits endothelial recovery and enhances plaque formation after arterial injury in ApoEdeficient mice. Arterioscler Thromb Vasc Biol. 2004;24(10):1891-1896. 
135. Strieter RM, Polverini PJ, Kunkel SL, Arenberg DA, Burdick MD, Kasper J, Dzuiba J, Van Damme J, Walz A, Marriott D, et al. The functional role of the ELR motif in CXC chemokine-mediated angiogenesis. J Biol Chem. 1995;270(45):27348-27357.

136. Hakim TS, Sugimori K, Camporesi EM, Anderson G. Half-life of nitric oxide in aqueous solutions with and without haemoglobin. Physiol Meas. 1996;17(4):267-277.

137. Liu X, Cheng Y, Zhang S, Lin Y, Yang J, Zhang C. A necessary role of miR-221 and miR-222 in vascular smooth muscle cell proliferation and neointimal hyperplasia. Circ Res. 2009;104(4):476-487.

138. Liu X, Cheng Y, Yang J, Xu L, Zhang C. Cell-specific effects of miR-221/222 in vessels: molecular mechanism and therapeutic application. J Mol Cell Cardiol. 2012;52(1):245-255.

139. Gerszten RE, Garcia-Zepeda EA, Lim YC, Yoshida M, Ding HA, Gimbrone MA, Jr., Luster AD, Luscinskas FW, Rosenzweig A. MCP-1 and IL-8 trigger firm adhesion of monocytes to vascular endothelium under flow conditions. Nature. 1999;398(6729):718-723.

140. Lukacs NW, Strieter RM, Elner V, Evanoff HL, Burdick MD, Kunkel SL. Production of chemokines, interleukin-8 and monocyte chemoattractant protein-1, during monocyte: endothelial cell interactions. Blood. 1995;86(7):2767-2773.

141. Akagawa KS. Functional heterogeneity of colony-stimulating factor-induced human monocyte-derived macrophages. Int J Hematol. 2002;76(1):27-34.

142. Shields JM, Christy RJ, Yang VW. Identification and characterization of a gene encoding a gut-enriched Kruppel-like factor expressed during growth arrest. $J$ Biol Chem. 1996;271(33):20009-20017.

143. Wei D, Gong W, Kanai M, Schlunk C, Wang L, Yao JC, Wu TT, Huang S, Xie K. Drastic down-regulation of Kruppel-like factor 4 expression is critical in human gastric cancer development and progression. Cancer Res. 2005;65(7):2746-2754.

144. Segre JA, Bauer C, Fuchs E. Klf4 is a transcription factor required for establishing the barrier function of the skin. Nat Genet. 1999;22(4):356-360.

145. Zhou G, Hamik A, Nayak L, Tian H, Shi H, Lu Y, Sharma N, Liao X, Hale A, Boerboom L, Feaver RE, Gao H, Desai A, Schmaier A, Gerson SL, Wang Y, Atkins GB, Blackman BR, Simon DI, Jain MK. Endothelial Kruppel-like factor 4 protects against atherothrombosis in mice. J Clin Invest. 2012;122(12):4727-4731.

146. Huang PL, Huang Z, Mashimo H, Bloch KD, Moskowitz MA, Bevan JA, Fishman MC. Hypertension in mice lacking the gene for endothelial nitric oxide synthase. Nature. 1995;377(6546):239-242.

147. Knowles JW, Reddick RL, Jennette JC, Shesely EG, Smithies O, Maeda N. Enhanced atherosclerosis and kidney dysfunction in eNOS(-/-)Apoe(-/-) mice are ameliorated by enalapril treatment. J Clin Invest. 2000;105(4):451-458.

148. Aso Y. Plasminogen activator inhibitor (PAI)-1 in vascular inflammation and thrombosis. Front Biosci. 2007;12:2957-2966.

149. Cherepanova OA, Pidkovka NA, Sarmento OF, Yoshida T, Gan Q, Adiguzel E, Bendeck MP, Berliner J, Leitinger N, Owens GK. Oxidized phospholipids induce type VIII collagen expression and vascular smooth muscle cell migration. Circ Res. 2009;104(5):609-618. 
150. Liu Y, Sinha S, McDonald OG, Shang Y, Hoofnagle MH, Owens GK. Kruppel-like factor 4 abrogates myocardin-induced activation of smooth muscle gene expression. $J$ Biol Chem. 2005;280(10):9719-9727.

151. Yoshida T, Kaestner KH, Owens GK. Conditional deletion of Kruppel-like factor 4 delays downregulation of smooth muscle cell differentiation markers but accelerates neointimal formation following vascular injury. Circ Res. 2008;102(12):1548-1557.

152. Thomas JA, Deaton RA, Hastings NE, Shang Y, Moehle CW, Eriksson U, Topouzis S, Wamhoff BR, Blackman BR, Owens GK. PDGF-DD, a novel mediator of smooth muscle cell phenotypic modulation, is upregulated in endothelial cells exposed to atherosclerosis-prone flow patterns. Am J Physiol Heart Circ Physiol. 2009;296(2):H442-452.

153. Bagi Z, Frangos JA, Yeh JC, White CR, Kaley G, Koller A. PECAM-1 mediates NOdependent dilation of arterioles to high temporal gradients of shear stress. Arterioscler Thromb Vasc Biol. 2005;25(8):1590-1595.

154. Chen Z, Tzima E. PECAM-1 is necessary for flow-induced vascular remodeling. Arterioscler Thromb Vasc Biol. 2009;29(7):1067-1073.

155. Gosling J, Slaymaker S, Gu L, Tseng S, Zlot CH, Young SG, Rollins BJ, Charo IF. MCP-1 deficiency reduces susceptibility to atherosclerosis in mice that overexpress human apolipoprotein B. J Clin Invest. 1999;103(6):773-778. 